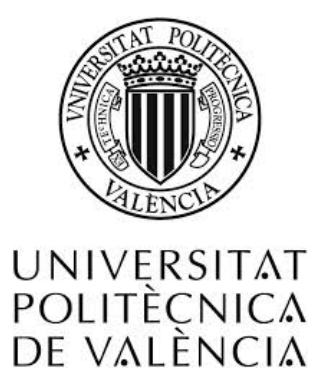

\title{
Topological and Computational Models for Fuzzy Metric Spaces via Domain Theory
}

\author{
Memoria presentada por \\ Luis A. Ricarte Moreno \\ para optar al Grado de Doctor por la \\ Universitat Politècnica de VAlènCIA \\ Dirigida por el Doctor \\ D. Salvador Romaguera Bonilla
}

Valencia, Octubre 2013 

D. Salvador Romaguera Bonilla, Catedrático del Departamento de Matemática Aplicada de la Universitat Politècnica de València

CERTIFICO: que la presente memoria "Topological and Computational Models for Fuzzy Metric Spaces via Domain Theory" ha sido realizada bajo mi dirección por D. Luis A. Ricarte Moreno, en el Departamento de Matemática Aplicada de la Universitat Politècnica de València, y constituye su tesis para optar al grado de Doctor por la Universitat Politècnica de València.

Y para que así conste, presento la referida tesis, firmando el presente certificado.

Valencia, Octubre de 2013

Fdo. Salvador Romaguera Bonilla 


\section{Agradecimientos}

Esta tesis no habría visto la luz, al menos con mi firma, sin la implicación

del profesor Salvador Romaguera. A él debo el haber llegado hasta el final. Muchas gracias.

Asimismo, agradezco a mi familia lo mucho que me ayudan cada día en el desempeño de mis deberes, también en la elaboración de esta tesis. VLMAG. DOG. 


\title{
RESUMEN ESPAÑOL
}

\author{
Luis A. Ricarte
}

Esta tesis doctoral está dedicada a investigar el problema de establecer conexiones entre la teoría de dominios y la teoría de los espacios métricos difusos (Fuzzy), en el sentido de Kramosil y Michalek, a través de la noción de bola formal, construyendo modelos topológicos y computacionales para espacios métricos (completos) difusos.

Los antecedentes de esta investigación son, principalmente, los conocidos artículos de A. Edalat y R. Heckmann [A computational model for metricspaces, Theoretical Computer Science 193 (1998), 53-73] y R. Heckmann [Approximation of metricspaces by partial metric spaces, Applied Categorical Structures 7 (1999), 71-83], donde los autores obtuvieron enlaces directos entre la teoría de dominios y la teoría de los espacios métricos -dos herramientas cruciales en el estudio de las semánticas denotacionales- utilizando bolas formales.

Puesto que cada métrica induce una métrica difusa (la llamada métrica difusa estándar), el problema de extender los resultados de Edalat y Heckmann al problema difuso surge de manera natural.

En nuestro estudio proponemos esencialmente dos aproximaciones diferentes. Para la primera, válida para aquellos espacios métricos difusos cuya t-norma continua es el mínimo, introducimos una nueva noción de completitud de un espacio métrico difuso, que nos permite construir un modelo (topológico) que incluye la teoría clásica como un caso particular. La segunda, válida para aquellos espacios métricos difusos cuya t-norma continua sea mayor o igual que la t-norma de Lukasiewicz, nos permite construir, entre otros resultados satisfactorios, un espacio casi-métrico difuso en el dominio continuo de las bolas formales, cuya restricción del conjunto de elementos maximales es isométrica al espacio métrico difuso dado. Así obtenemos un modelo computacional para los espacios métricos difusos completos. 
Asimismo probamos algunos nuevos teoremas de punto fijo en espacios métricos difusos completos con versiones para el caso intuicionista y el caso ordenado, respectivamente.

Finalmente, discutimos el problema de extender los resultados obtenidos al problema asimétrico. 


\title{
RESUMEN VALENCIANO
}

\author{
Luis A. Ricarte Moreno
}

Esta tesi doctoral està dedicada a investigar el problema d'establir connexions entre la teoria de dominis i la teoria dels espais mètrics difusos (Fuzzy), en el sentit de Kramosil i Michalek, mitjançant la noció de bola formal, construint models topològics i computacionals per a espais mètrics (complets) difusos.

Els antecedents d'esta investigació són, principalment, els coneguts articles de A. Edalat i R. Heckmann [A computational model for metric spaces, Theoretical Computer Science 193 (1998), 53-73] i R. Heckmann [Approximation of metric spaces by partial metric spaces, Applied Categorical Structures 7 (1999), 71-83], on els autors obtingueren enllaços directes entre la teoria de dominis i la teoria dels espais mètrics -dues ferramentes crucials en l'estudi de les semàntiques denotacionals- utilitzant boles formals.

Ja que cada mètrica induix una mètrica difusa (l'anomenada mètrica difusa estàndard), el problema d'extendre els resultats d' Edalat i Heckmann al problema difuso sorgix de manera natural.

$\mathrm{Al}$ nostre estudi proposem, essencialment dues aproximacions diferents. Per a la primera, vàlida per aquells espais mètrics difusos quan una t-norma contínua és el mínim, introduïm una nova noció d'un espai mètric difús complet, que ens permet construir un model (topològic) que inclou la teoria clàssica com un cas particular. La segona, vàlida per aquells espais mètrics difusos quan una t-norma contínua siga major o igual que la t-norma de Lukasiewicz, ens permet construir, entre altres resultats satisfactoris, un espai quasi-mètric difús en el domini continu de les boles formals, ja que la restricció del conjunt d'elements maximals és isomètrica a l'espai mètric difús donat. Així obtenim un model computacional per als espais mètrics difusos complets. 
Així mateix probarem alguns nous teoremes de punt fixe en espais mètrics complets amb versions per al cas intuïcionista i el cas ordenat, respectivament.

Finalment, discutim el problema d'estendre els resultats obtinguts al problema asimètric. 


\title{
RESUMEN INGLES
}

\author{
Luis A. Ricarte
}

This doctoral thesis is devoted to investigate the problem of establishing connections between Domain Theory and the theory of fuzzy metric spaces, in the sense of Kramosil and Michalek, by means of the notion of a formal ball, and then constructing topological and computational models for (complete) fuzzy metric spaces.

The antecedents of this research are mainly the well-known articles of A. Edalat and R. Heckmann [A computational model for metric spaces, Theoretical Computer Science 193 (1998), 53-73], and R. Heckmann [Approximation of metric spaces by partial metric spaces, Applied Categorical Structures 7 (1999), 71-83], where the authors obtained nice and direct links between Domain Theory and the theory of metric spaces - two crucial tools in the study of denotational semantics - by using formal balls.

Since every metric induces a fuzzy metric (the so-called standard fuzzy metric), the problem of extending Edalat and Heckmann's works to the fuzzy framework arises in a natural way.

In our study we essentially propose two different approaches. For the first one, valid for those fuzzy metric spaces whose continuous t-norm is the minimum, we introduce a new notion of fuzzy metric completeness (the so-called standard completeness) that allows us to construct a (topological) model that includes the classical theory as a special case. The second one, valid for those fuzzy metric spaces whose continuous t-norm is greater or equal than the Lukasiewicz t-norm, allows us to construct, among other satisfactory results, a fuzzy quasi-metric on the continuous domain of formal balls whose restriction to the set of maximal elements is isometric to the given fuzzy metric. Thus we obtain a computational model for complete fuzzy metric spaces. 
We also prove some new fixed point theorems in complete fuzzy metric spaces with versions to the intuitionistic case and the ordered case, respectively.

Finally, we discuss the problem of extending the obtained results to the asymmetric framework. 


\section{Contents}

1 General Introduction, and Preliminaries 3

1.1 General Introduction . . . . . . . . . . . . . . . . . . . 3

1.2 Preliminaries ................... . . . 5

2 Background 33

2.1 Fuzzy metric and fuzzy quasi-metric spaces . . . . . . . . 33

2.2 Domain Theory . . . . . . . . . . . . . . . . . 39

3 A domain theoretic approach to fuzzy metric spaces based on the standard fuzzy metric 43

3.1 Introduction . . . . . . . . . . . . . . . . . . . 43

3.2 The poset $\left(\mathbf{B} X, \sqsubseteq_{M}\right)$ of formal balls of a fuzzy metric space $(X, M, \wedge) \ldots \ldots \ldots \ldots \ldots \ldots$

3.3 Complete fuzzy metric spaces of type $(X, M, \wedge)$ and the dcpo $\left(\mathbf{B} X, \sqsubseteq_{M}\right) \ldots \ldots \ldots$. . . . . . . . . . . . . 47

3.4 Characterizing $\left(\mathbf{B} X, \sqsubseteq_{M}\right)$ as a domain: Standard complete fuzzy metric spaces . . . . . . . . . . . . . . . . . 54

3.5 A notion of computational model for complete fuzzy metric

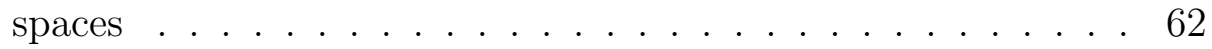

4 A computational model for complete fuzzy metric spaces 63

4.1 Introduction . . . . . . . . . . . . . . . . . 63 
4.2 The poset $(\mathbf{B} X, \sqsubseteq)$ of formal balls of a fuzzy metric space $(X, M, *)$ with $* \geq *_{L} \ldots \ldots$. . . . . . . . . . . 64

4.3 Relations between fuzzy metric spaces and properties of Domain Theory via the poset $(\mathbf{B} X, \sqsubseteq)$. . . . . . . . . . . 69

4.4 A computational model for complete fuzzy metric spaces $(X, M, *)$ with $* \geq *_{L} \ldots \ldots \ldots$. . . . . . . . . . . . . . . . . 72

5 Fixed point theorems for $\varphi$-contractions on fuzzy metric spaces $\begin{array}{ll}\text { with application to the intuitionistic setting } & 81\end{array}$

5.1 Introduction . . . . . . . . . . . . . . . 81

5.2 Fixed point theorems for complete fuzzy metric spaces . . . . 82

5.3 Application to intuitionistic fuzzy metric spaces . . . . . . . 89

5.4 A version for ordered fuzzy metric spaces . . . . . . . . . . . 94

$\begin{array}{lll}6 & \text { The fuzzy quasi-metric case } & 99\end{array}$

6.1 Introduction . . . . . . . . . . . . . . . . . . . 99

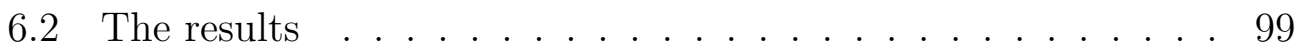

$\begin{array}{ll}\text { Bibliography } & 107\end{array}$ 


\section{Chapter 1}

\section{General Introduction, and Preliminaries}

\subsection{General Introduction}

The origin of Domain Theory is in the work of Danna Scott in the late 1960s, who used partially ordered structures on function spaces to the study of denotational semantics for programming languages, in joint paper with Christopher Strachey ([74]). From then, various authors have developed the mathematical theory of domains (see, for instance, [2, 25, 75, 26]).

Since metric spaces also provide an efficient tool in the study of denotational semantics (see e.g. [10]), several mathematicians have investigated the natural problem of establishing links between metric spaces, and other topological structures, and Domain Theory.

In this direction, an important first result was obtained by Lawson who characterized in [44] Polish spaces (i.e., complete separable metric spaces) in terms of $\omega$-domains (see Chapter 2 for the notion of an $\omega$-domain). It is interesting to recall that, previously, Edalat ([18, 19]) constructed domain theoretic models for metrizable locally compact spaces, and applied such 
constructions to dynamical systems, measures, iterated functions systems, and fractals.

In their celebrated paper [20], Edalat and Heckmann presented nice, direct and very visual connections between Domain Theory and the theory of metric spaces by using the notion of a (closed) formal ball. In this way, they proved, among other interesting results, that a metric space is complete if and only if its poset of formal balls is a domain, and constructed a topological model in the sense of Martin (see Chapter 2) for any complete metric space.

Later on, Heckmann ([36]) constructed a suitable bicomplete quasi-metric on the domain of formal balls of a complete metric space which provides an efficient computational model for metric spaces.

Further contributions to the construction of models for metric spaces and other related structures by using domains may be found in $[5,21,26,41,61$, $62,63,66,67,68,71,78,79]$.

Motivated on the one hand by the researches about the construction of models for metric spaces and other related structures via domains cited above, and on the other hand by the well-known fact that every metric on a set induces in a natural way a fuzzy metric in the sense of Kramosil and Michalek (see Chapter 2 for details), we shall investigate the problem of establishing relationships between the theory of (complete) fuzzy metric spaces and Domain Theory.

Thus, in Section 1.2 of this chapter we recall some basic properties and facts about fuzzy sets and fuzzy logic, and their origins.

In Chapter 2 we recall several pertinent concepts and properties on fuzzy metric spaces and Domain Theory which will be useful in the rest of the thesis.

Chapters 3 and 4 are devoted to study (complete) fuzzy metric spaces from a domain theoretic approach. In Chapter 3 this is done taking as the starting point the construction of the standard fuzzy metric of a given metric 
space. The domain of formal balls constructed in this way is characterized by using a more general notion of fuzzy completeness, called standard fuzzy

completeness. This approach, which is valid for those fuzzy metric spaces whose continuous t-norm is the minimum, allows us to deduce the classical theory of Edalat and Heckmann as a special case.

In Chapter 4 we adapt to the fuzzy setting the poset structure given in [20]. This approach is valid for those fuzzy metric spaces whose continuous t-norm is greater or equal than the Lukasiewicz t-norm, and allows us to construct, among other satisfactory results, a fuzzy quasi-metric on the domain of formal balls whose restriction to the set of maximal elements is isometric to the given fuzzy metric. Thus we obtain a computational model for complete fuzzy metric spaces.

Chapter 5 deals with fixed point theorems in fuzzy metric spaces. Thus, in Section 5.2 we obtain two fixed point theorems for a kind of $\varphi$-contractive mappings on complete fuzzy metric spaces from which we easily deduce, in Section 5.3, fixed point results for complete intuitionistic fuzzy metric spaces. Such results improve in several directions the main fixed point theorem of [38], and also provide a simplified proof of it. Finally, in Section 5.4 we present a version for ordered complete fuzzy metric spaces of the main fixed point theorem obtained in Section 5.2.

In Chapter 6 we explore the extension of the results obtained in Chapters 3,4 and 5, to the context of fuzzy quasi-metric spaces.

\subsection{Preliminaries}

Everything is vague to a degree you do not realize till you have tried to make it precise (Bertrand Russell).

In 1942 the austrian mathematician Karl Menger, while serving as a professor at the University of Notre Dame in Chicago, introduced the concept 
of probabilistic metric space. Given a set $X$, Menger proposed to associate a distribution function $F_{x y}$ such that for each pair of elements $x, y$ of $X$ and each $t, F_{x y}(t)$ is a number belonging to the interval $[0,1]$.

It was interpreted as the probability that the distance between $x$ and $y$ is less than $t$. Thus, he achieved some flexility in determining which points belongs to a set: the degree of belonging is established by the distribution function.

In the following years this concept was advanced in trying to generalize the situations where there is no certainty that an item belongs to a set. Likelihood function is passed to a situation known as fuzzy uncertainty.

Kramosil and Michalek ([42]) successfully reformulated probablistic metric spaces in the fuzzy setting, introducing their celebrated notion of a fuzzy metric space (see Chapter 2) which will be used in this thesis.

The concept of fuzzy logic was introduced by the azerbaijan professor of the University of California at Berkley, Lotfi Zadeh, in an article published in 1965 and titled "Fuzzy Sets". Zadeh proposed in this work the concept partial membership, or in other words, the existence of a certain degree of membership of an element to a set.

The next comments are based on:

http://www.calvin.edu/ pribeiro/othrlnks/Fuzzy/home.htm

and on the work of Prof. $\mathrm{M}^{a}$ José Gacto, Teoría de Sistemas, Control de Sistemas y Sistemas Inteligentes.

Although at the beginning of the introduction of fuzzy concepts the underlying mathematics where not fully developed, and fuzzy logic was only partially well accepted, especially in academic groups, it was recognized as a very natural way of thinking and processing information, and applications started to be developed.

To understand the objections made to fuzzy logic in its early days, it is interesting to review some quotes from those days. Thus, and according 
to Professor William Kahan UC Berkeley (colleague of Lotfi Zadeh at UC Berkeley) :

"Fuzzy theory is wrong, wrong, and pernicious. What we need is more logical thinking, not less. The danger of fuzzy logic is that it will encourage the sort of imprecise thinking that has brought us so much trouble. Fuzzy logic is the cocaine of science."

According to Professor Rudolf Kalman, University of Florida:

"Fuzzification is a kind of scientific permissiveness. It tends to result in socially appealing slogans unaccompanied by the discipline of hard scientific work and patient observation"

and according to Professor Myron Tribus, on hearing of the fuzzy-logic control of the Sendai subway system IEEE Institute:

"Fuzziness is probability in disguise. I can design a controller with probability that could do the same thing that you could do with fuzzy logic." .

To understand the concept behind the Fuzzy Logic the following text from http://www.calvin.edu/ pribeiro/othrlnks/Fuzzy/home.htm is very appropriate:

"The way that people think is inherently fuzzy. The way that we perceive the world is continually changing and cannot always be defined in true or false statements. Take for example the set of all the apples and all the apple cores in the world. Now take one of those apples; it belongs to the set of all apples. Now take a bite out of that apple; it is still an apple right? If so, it still belongs to the set of apples. After several more bites have been taken and you are left with an apple core and it belongs to the set of apple cores. At what point did the apple cross over from being an apple to being an apple core? What if you could get one more bite out of that apple core, does that move it into a different set?

The definition of the apple and apple core sets are too strictly defined when looking at the process of eating an apple. The area between the two sets 
is not clearly defined since the object cannot belong to the set of apples and apple cores because, by definition, an apple core is NOT an apple. The sets defining apples and apple cores need to be redefined as fuzzy sets.

A fuzzy set allows for its members to have degrees of membership. If the value of 1 is assigned to objects entirely within the set and $a 0$ is assigned to objects outside of the set, then any object partially in the set will have a value between 0 and 1 . The number assigned to the object is called its degree of membership in the set. So an apple with one bite out of it may have a degree of membership of 0.9 in the set of apples. This does not mean that it has to have a degree of membership of 0.1 in the set of apple cores though. However as the apple is eaten it looses its membership in the fuzzy set of apples and gains membership in the fuzzy set of apple cores."

\section{Fuzzy Sets:}

In mathematics a set, by definition, is a collection of things that belong to some definition. Any item either belongs to that set or does not belong to that set. Let us look at another example: The set of tall men. We shall say that people taller than or equal to $182 \mathrm{~cm}$ are tall. This set can be represented graphically as follows:

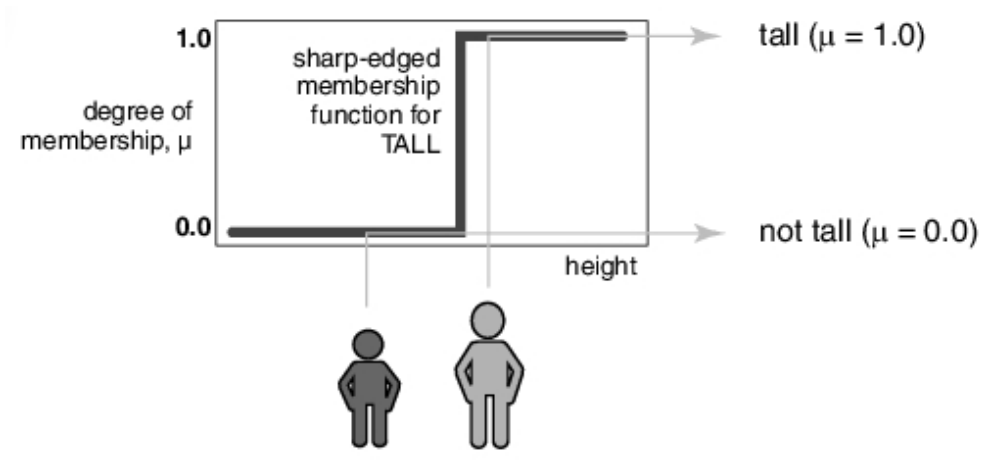

The function shown above describes the membership of the 'tall' set, you are either in it or you are not in it. This sharp edged membership functions 
works nicely for binary operations and mathematics, but it does not work as nicely in describing the real world. The membership function makes no distinction between somebody who is $185 \mathrm{~cm}$ and someone who is $210 \mathrm{~cm}$, they are both simply tall. Clearly there is a significant difference between the two heights. The other side of this lack of distinction is the difference between a $180 \mathrm{~cm}$ and $182 \mathrm{~cm}$ man. This is only a difference of two $\mathrm{cm}$, however this membership function just says one is tall and the other is not tall.

The fuzzy set approach to the set of tall men provides a much better representation of the tallness of a person. The set, shown below, is defined by a continuously inclining function.

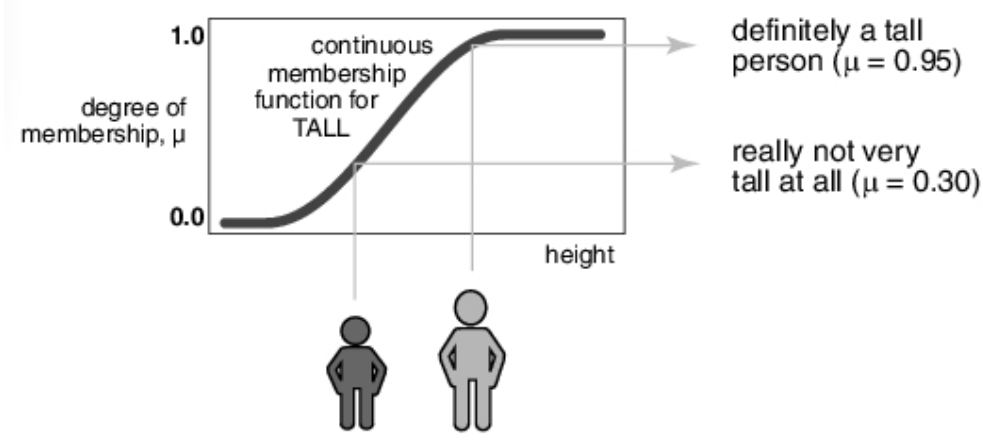

The membership function defines the fuzzy set for the possible values underneath of it on the horizontal axis. The vertical axis, on a scale of 0 to 1 , provides the membership value of the height in the fuzzy set. So for the two people shown above the first person has a membership of 0.3 and so is not very tall. The second person has a membership of 0.95 and so he is definitely tall. He does not, however, belong to the set of tall men in the way that bivalent sets work; he has a high degree of membership in the fuzzy set of tall men.

Fuzzy logic was developed in order to make judgments, reasoning, representations, etc., based on inaccurate, vague knowledge, expressed with variables that are excerpts from the natural speech. The following briefly 
introduce the main concepts of logic and fuzzy sets.

One of the first concept is that of the fuzzy set. For example, a clear set (classic) $A$ might be: Real numbers belonging to the interval $X=[1,4]$ which values are between 2 and 3 . In this case the characteristic function or membership function $\mu_{A}: X \rightarrow\{0,1\}$ of the set $A$ is:

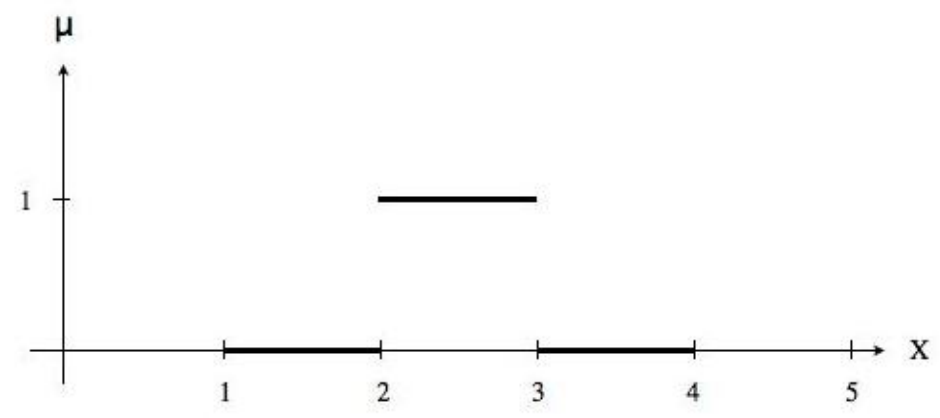

In this case is perfectly clear what the function $A$ means and there is not any difficulty to understand its behaviour because we are using just numbers with no relation to the real life. However, if we change the example, and talk, for instance, about the set of the people considered young, then everything change. Let's have a look to this set using a clear set. The membership function might be:

$$
\mu_{B}(x)=\left\{\begin{array}{lll}
1 & \text { if } & x \leq 30 \\
0 & \text { if } & x>30
\end{array}\right.
$$

That mens that a 29 years old man should be considered young, but another one 31 years old shouldn't. Let's consider now a fuzzy set B formed by the age of a young person. In this case, the characteristic function of the set might be:

$$
\mu_{B}: X \rightarrow[0,1]
$$

where 


$$
\mu_{B}(x)= \begin{cases}1 & \text { if } 0 \leq x \leq 20 \\ 40-x / 20 & \text { if } 20<x \leq 40 \\ 0 & \text { if } 40<x\end{cases}
$$

The following chart shows the membership function $\mu_{B}$

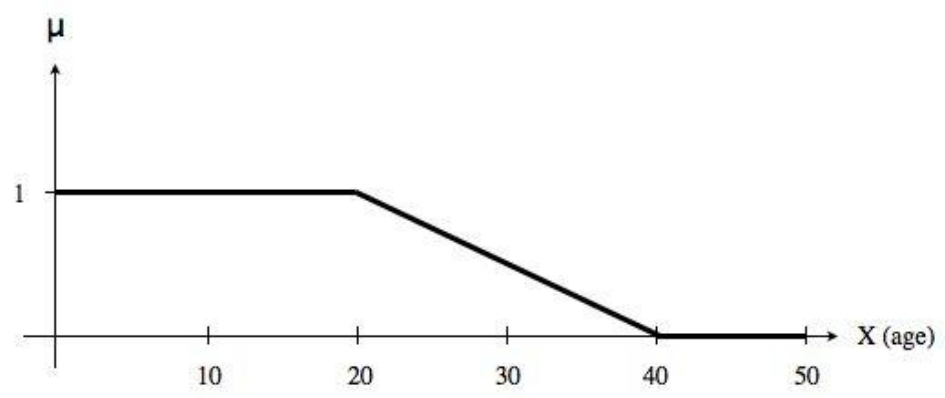

In this case $\mu_{B}(19)=1$, i.e. a young 19 years old man belongs completely to the set of young people; but for example a 35 years old man has a membership degree equal to $\mu_{B}(30)=0,5$ what could be interpreted saying that is quite young but starts its maturity.

Let's a have look to the the definition of a Fuzzy Set established by Lofti A. Zadeh in his famous paper "Fuzzy sets", Information and Control 8 (1965), 338-353.

Let $X$ be a space of points, with a generic element of $X$ denoted by $x$. A fuzzy set $A$ in $X$ is characterized by a membership function $f_{A}(x)$ which associates with each point in $X$ a real number in the interval $[0,1]$, with the values of $f_{A}(x)$ at $x$ representing the "grade of membership" of $x$ in $A$. Thus, the nearer the value of $f_{A}(x)$ to unity, the higher the grade of membership of $x$ in $A$.

Some examples of fuzzy sets could be:

A) Set of all trains crossing near a certain road.

B) Set of real numbers close to 1 . 
C) Set of elderly people.

D) Set of supreme quality bottled wines.

Membership functions for fuzzy sets can be defined in any number of ways as long as they follow the rules of the definition of a fuzzy set. The shape of the membership function used defines the fuzzy set and so the decision on which type to use is dependant on the purpose. The membership function choice is the subjective aspect of fuzzy logic, it allows the desired values to be interpreted appropriately.

[see http://www.dma.fi.upm.es/java/fuzzy/fuzzyinf/funpert_en.htm]

Some of the most common membership functions are as follows:

- Triangular function: defined by a lower limit $a$, an upper limit $b$, and a value $m$, where $a<m<b$ :

$$
\mu(x)= \begin{cases}0 & \text { if } x \leq a \\ (x-a) /(m-a) & \text { if } a<x \leq m \\ (b-x) /(b-m) & \text { if } m<x<b \\ 0 & \text { if } x \geq b\end{cases}
$$

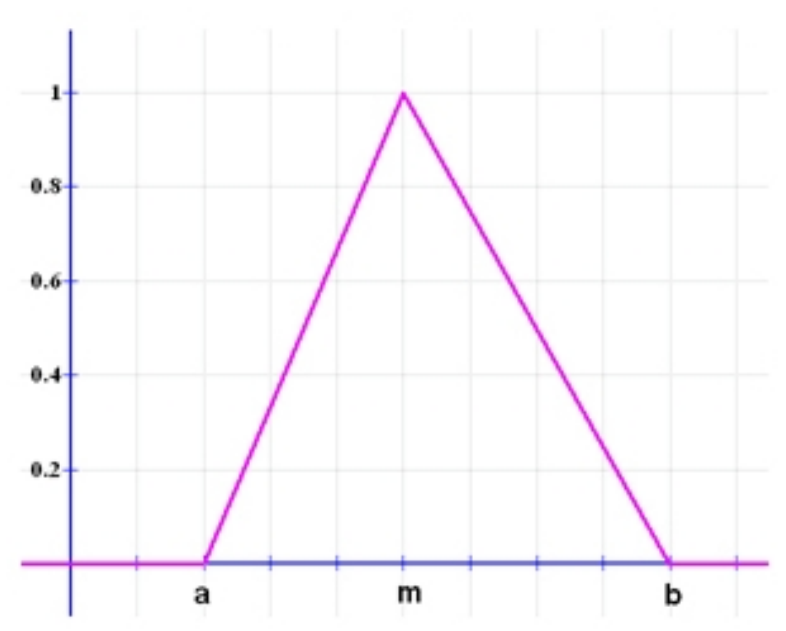


- Trapezoidal function: defined by a lower limit $a$, an upper limit $d$, a lower support limit $b$, and an upper support limit $c$, where $a<b<c<$ $d$ :

$$
\mu(x)= \begin{cases}0 & \text { if } \quad(x<a) \text { or }(x>d) \\ (x-a) /(b-a) & \text { if } a \leq x \leq b \\ 1 & \text { if } b \leq x \leq c \\ (d-x) /(d-c) & \text { if } c \leq x \leq d\end{cases}
$$

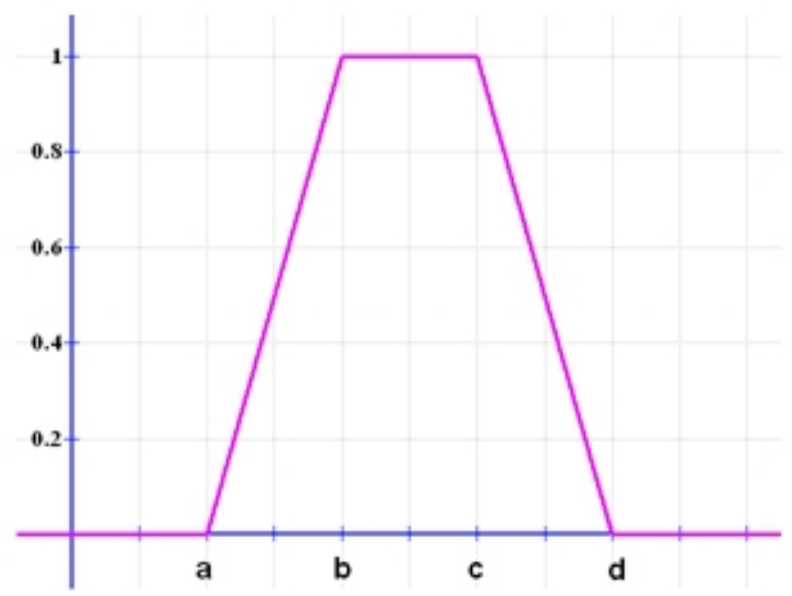

There are two special cases of a trapezoidal function, which are called R-functions and L-functions.

- Trapezoidal R-function: with parameters $a=b=-\infty$

$$
\mu(x)= \begin{cases}0 & \text { if } \quad x>d \\ (d-x) /(d-c) & \text { if } c \leq x \leq d \\ 1 & \text { if } x<c\end{cases}
$$




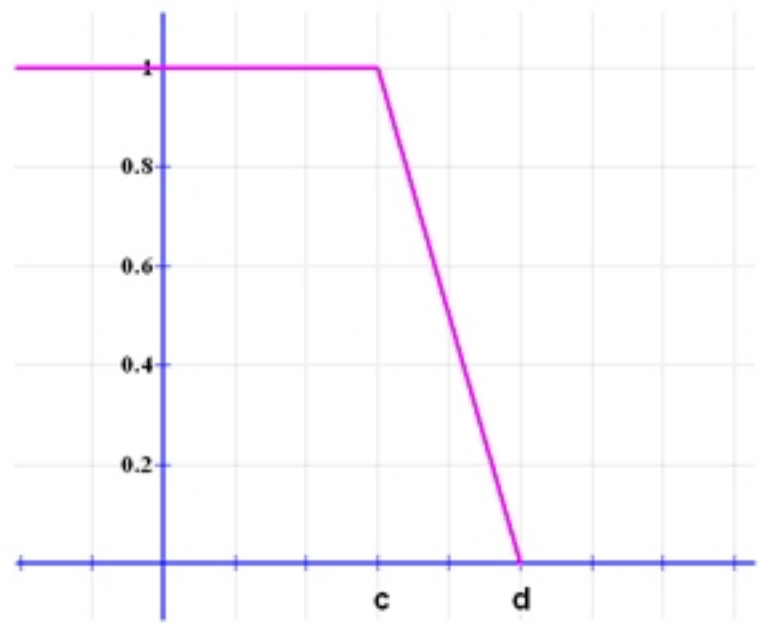

- Trapezoidal L-function: with parameters $c=d=+\infty$

$$
\mu(x)= \begin{cases}0 & \text { if } x<a \\ (x-a) /(b-a) & \text { if } a \leq x \leq b \\ 1 & \text { if } x>b\end{cases}
$$

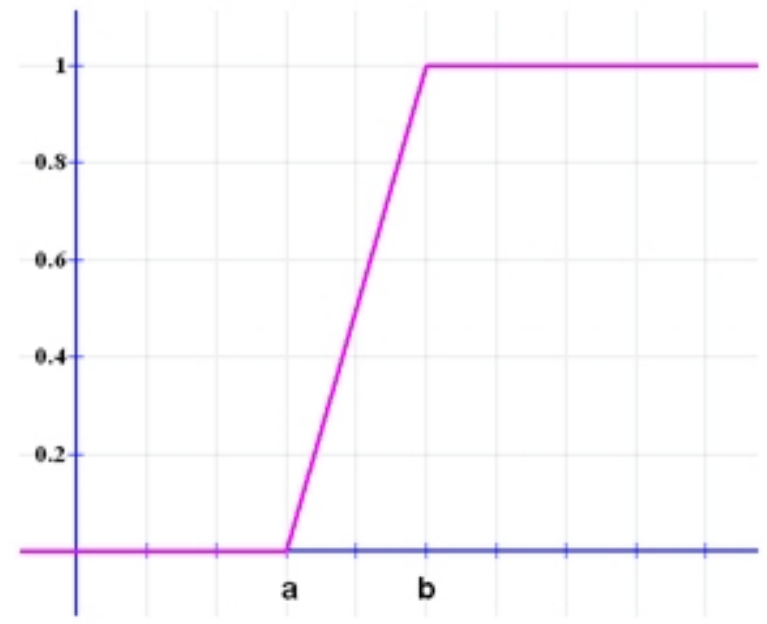

- Gaussian function: defined by a central value $m$ and a standard deviation $k>0$. The smaller $k$ is, the narrower the "bell": 


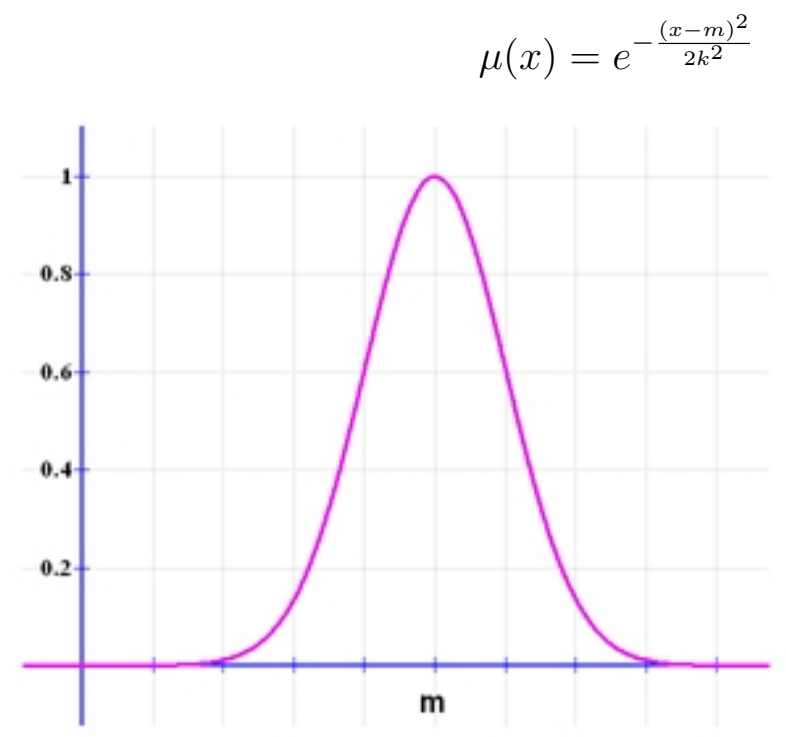

The main properties that characterize a fuzzy set is listed below:

- Support: set of elements whose degree of membership is nonzero:

$$
\operatorname{Support}(A)=\left\{x \mid \mu_{A}(x)>0, x \in X\right\}
$$

- Height: higher degree of membership of an element:

$$
\operatorname{Height}(A)=\max \left\{h \mid h=\mu_{A}(x), x \in X\right\}
$$

- Kernel (Maximal Elements): set of all elements whose membership degree equals to 1 :

$$
\operatorname{Kernel}(A)=\left\{x \mid \mu_{A}(x)=1, x \in X\right\}
$$

- Normal fuzzy set: every fuzzy set whose Height equals to 1. 
- Convex fuzzy set:

$$
\mu_{A}(\lambda x+(1-\lambda) y) \geq \min \left(\mu_{A}(x), \mu_{B}(y)\right)
$$

where $x, y \in X$ and $\lambda \in[0,1]$.

\section{Relations and Operations in Fuzzy Sets:}

A relationship between classic sets may be considered a set of n-tuples that satisfy a certain condition: for example, considering the condition "greater than" between the elements of two classic sets, this relationship would include all pairs of elements from both sets that satisfy the condition:

$$
\text { "Greater than" }=\{(x, y) \mid x \geq y, x \in A, y \in B\}
$$

A relationship between fuzzy sets is a fuzzy subset of the cartesian product of the two sets which includes all those elements that satisfy the condition in some degree. For example, if we consider the ratio $R=$ "near to", we have:

$$
\begin{aligned}
& A=\{1,2,3,4\} \\
& R: A \times A \rightarrow \mu_{R}=\left\{\begin{array}{lll}
1 & \text { if } & x=y \\
0,9 & \text { if } & |x-y|=1 \\
0,7 & \text { if } & |x-y|=2 \\
0,3 & \text { if } & |x-y|=3
\end{array}\right.
\end{aligned}
$$




\begin{tabular}{c|c|c|c|c|}
\multicolumn{1}{c|}{} & \multicolumn{4}{c|}{$\mathrm{x}$} \\
\cline { 2 - 6 } \multicolumn{1}{c|}{$\mathrm{y}$} & 1 & 2 & 3 & 4 \\
\hline 1 & 1 & 0,9 & 0,7 & 0,3 \\
\hline 2 & 0,9 & 1 & 0,9 & 0,7 \\
\hline 3 & 0,7 & 0,9 & 1 & 0,9 \\
\hline 4 & 0,3 & 0,7 & 0,9 & 1 \\
\hline
\end{tabular}

Operations with fuzzy sets are an application of operations to fuzzy logic.

For example, we may list the following for every $x \in X$ :

- Equality:

$$
A=B \Leftrightarrow \mu_{A}(x)=\mu_{B}(x) .
$$

- Inclusion:

$$
A \subseteq B \Leftrightarrow \mu_{A}(x) \leq \mu_{B}(x)
$$

- Union:

$$
\mu_{A \cup B}=\max \left(\mu_{A}(x), \mu_{B}(x)\right) .
$$

- Intersection:

$$
\mu_{A \cap B}=\min \left(\mu_{A}(x), \mu_{B}(x)\right) .
$$

There are generalizations of those operations to be used in the subsequent mathematical development: 
- T-norm: generalizes the concept of fuzzy sets intersection:

$$
T:[0,1] \times[0,1] \rightarrow[0,1] \mid \mu_{A \cap B}=T\left(\mu_{A}(x), \mu_{B}(x)\right)
$$

And satisfies the following properties:

i) Commutativity: $T(a, b)=T(b, a)$

ii) Associativity: $T(a, T(b, c))=T(T(a, b), c)$

iii) Monotonicity: $T(a, b) \geq T(b, c)$ if $a \geq c$ and $b \geq d$

iv) Identity element: $T(a, 1)=a$

Examples of t-norms:

- Standard intersection (Minimum t-norm or Gödel t-norm):

$$
T(a, b)=\min \{a, b\}
$$

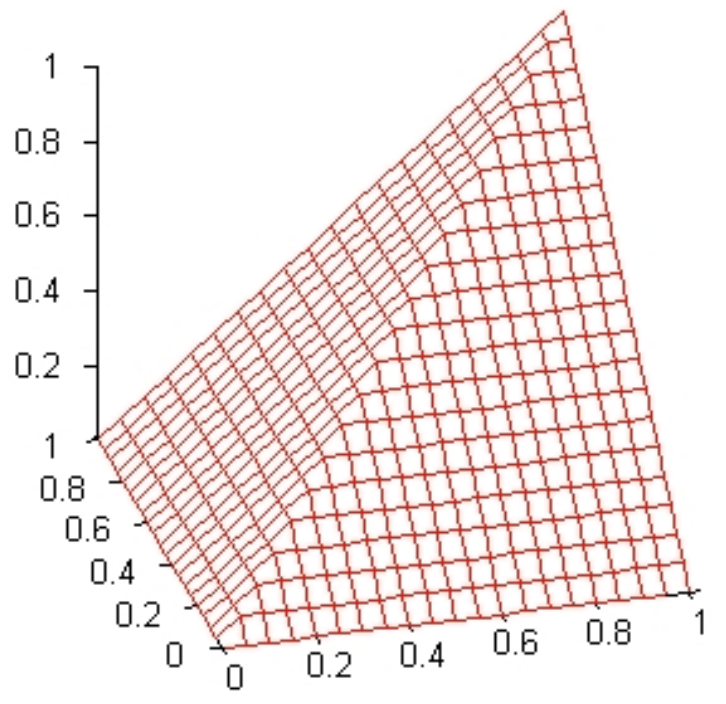


- Product t-norm:

$$
T(a, b)=a \cdot b
$$

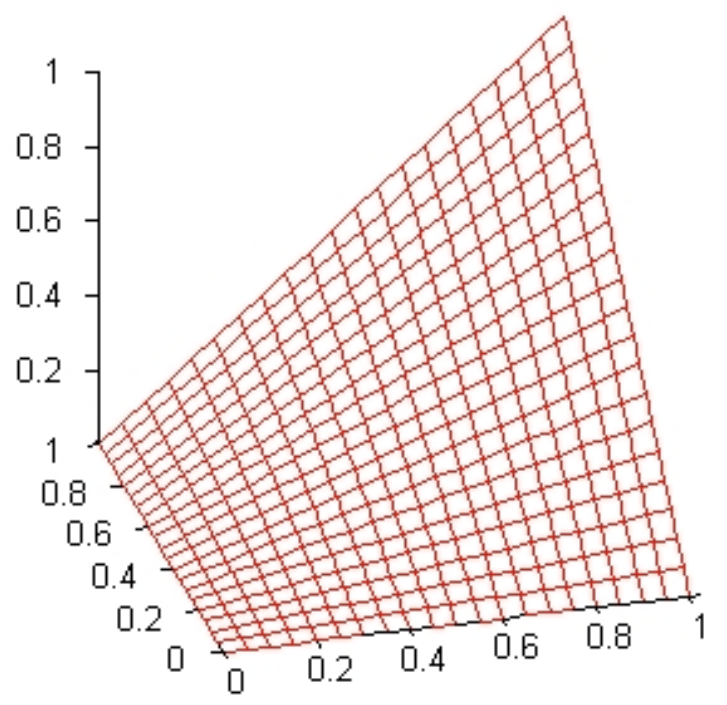

- Bounded difference (Lukasiewicz t-norm):

$$
T(a, b)=\max \{0, a+b-1\}
$$




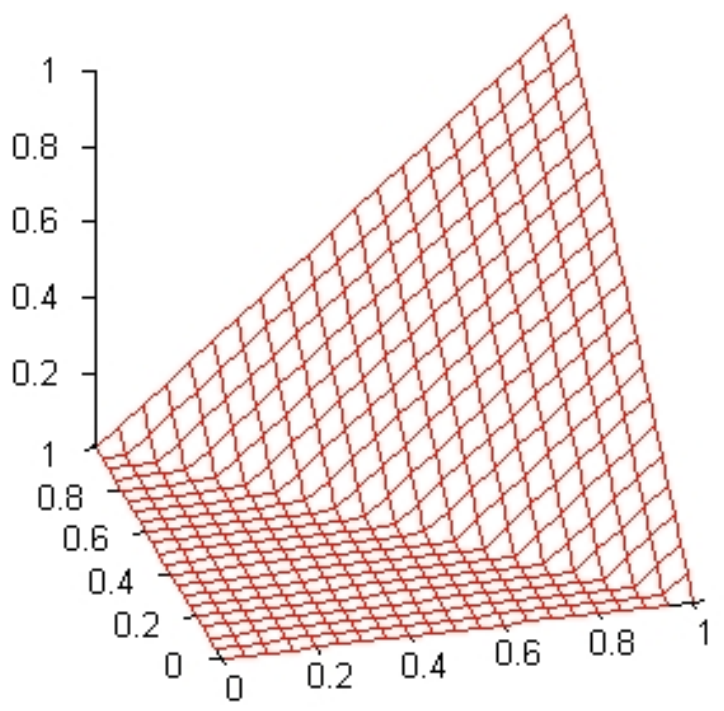

- Drastic t-norm:

$$
T(a, b)=\left\{\begin{array}{lll}
b & \text { if } & a=1 \\
a & \text { if } & b=1 \\
0 & \text { otherwise } &
\end{array}\right.
$$




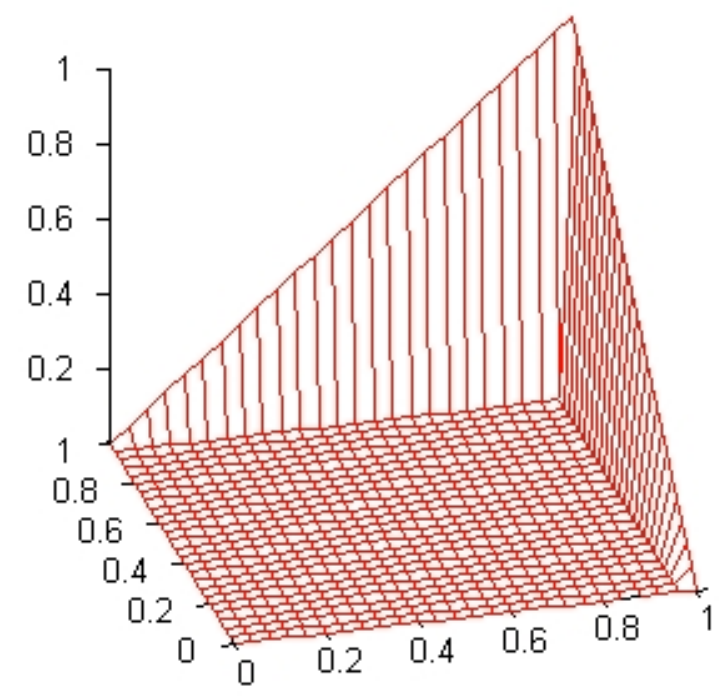

- T-conorm (also called s-norm): generalizes the concept of fuzzy sets intersection:

$$
S:[0,1] \times[0,1] \rightarrow[0,1] \mid \mu_{A \cup B}=S\left(\mu_{A}(x), \mu_{B}(x)\right)
$$

And satisfies the following properties:

i) Commutativity: $S(a, b)=S(b, a)$

ii) Associativity: $S(a, S(b, c))=S(S(a, b), c)$

iii) Monotonicity: $S(a, b) \leq S(b, c)$ if $a \leq c$ and $b \leq d$

iv) Identity element: $S(a, 0)=a$

Examples of t-conorms:

- Maximum t-conorm (dual to the minimum t-norm): 


$$
S(a, b)=\max \{a, b\}
$$

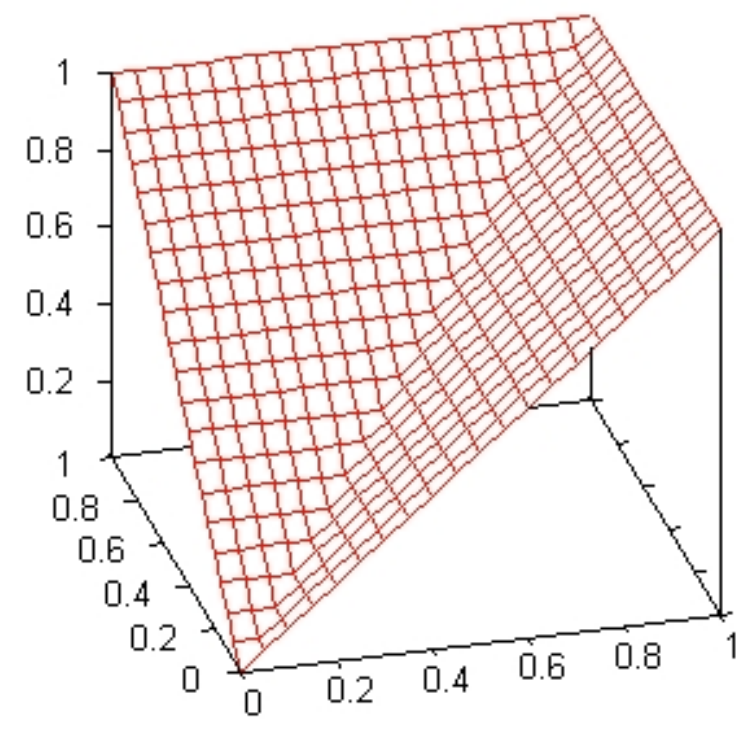

- Probabilistic sum (dual to the product t-norm):

$$
S(a, b)=a+b-a \cdot b
$$




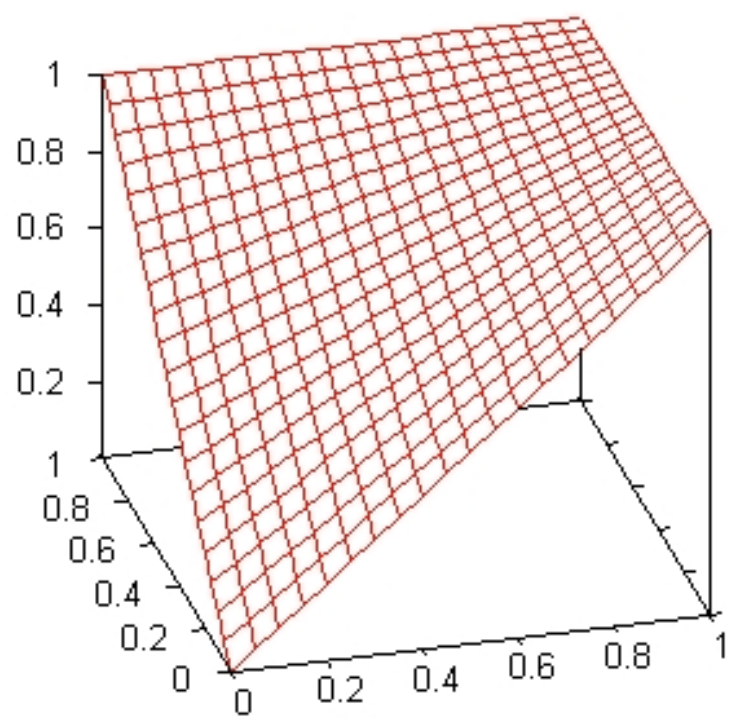

- Bounded sum (dual to the Lukasiewicz t-norm):

$$
S(a, b)=\min \{0, a+b-1\}
$$

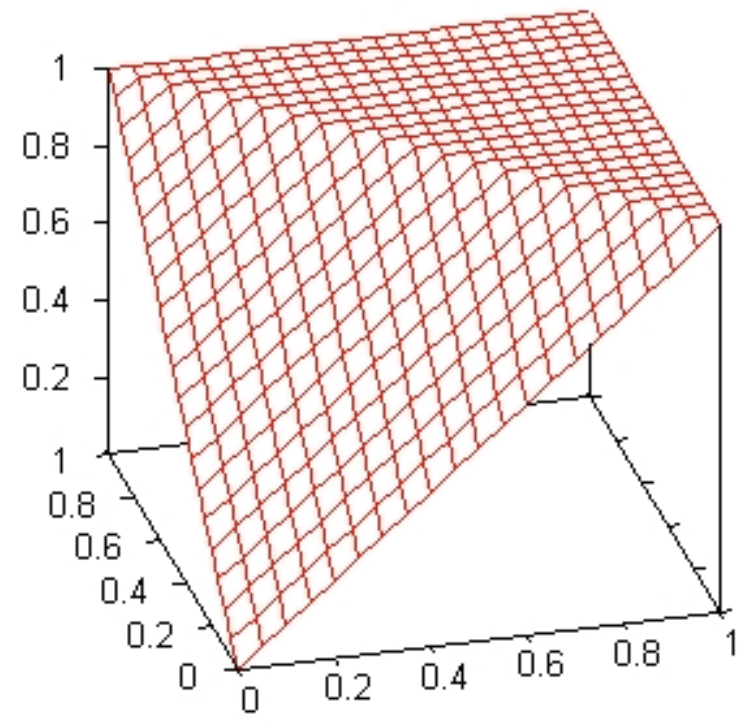


- Drastic t-conorm:

$$
S(a, b)=\left\{\begin{array}{lll}
b & \text { if } & a=0 \\
a & \text { if } & b=0 \\
0 & \text { otherwise } &
\end{array}\right.
$$

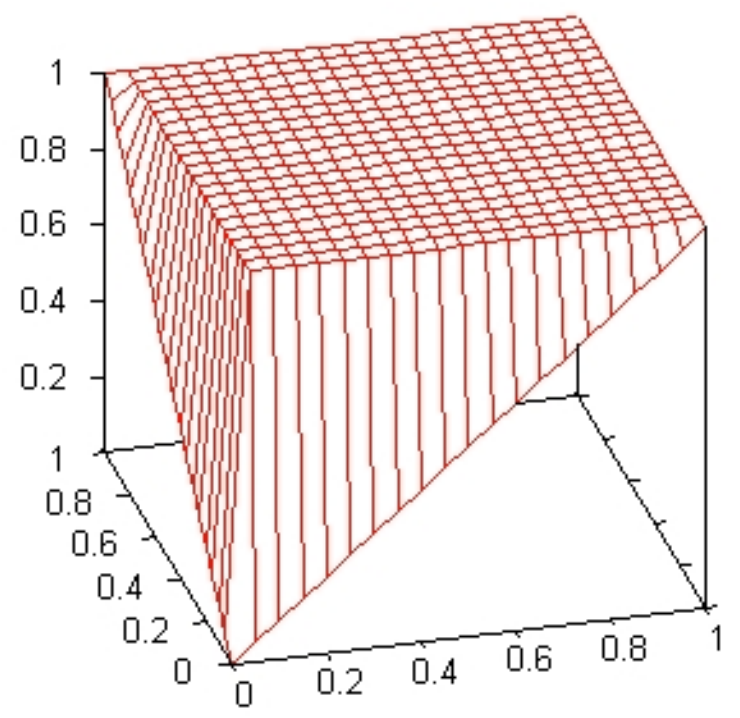

\section{Linguistic Variables, Fuzzy Variables and Fuzzy Rules:}

In papers published in 1973 and 1975, Professor Zadeh introduced the concept of linguistic variable and fuzzy variable, while he was developing its application in engineering.

The linguistic variables are variables whose possible values are linguistic terms that become determined by fuzzy sets. They have the property to provide a gradual transition between states, and due to the way they are defined are much better for working with situations of uncertainty that the crisp variables. 
A linguistic variable is defined by a set of parameters that can be described as follows:

$$
(V, T, X, G, M)
$$

where

$V$ : Variable name.

$T$ : Set of linguistic terms.

$X$ : Universe of discourse.

$G$ : syntactic rule for generating linguistic terms.

M: semantic rule which associates with each linguistic term $t$ its meaning $m(t)$, where $m(t)$ is a fuzzy set in $X$.

The following diagram shows the elements of a linguistic variable:

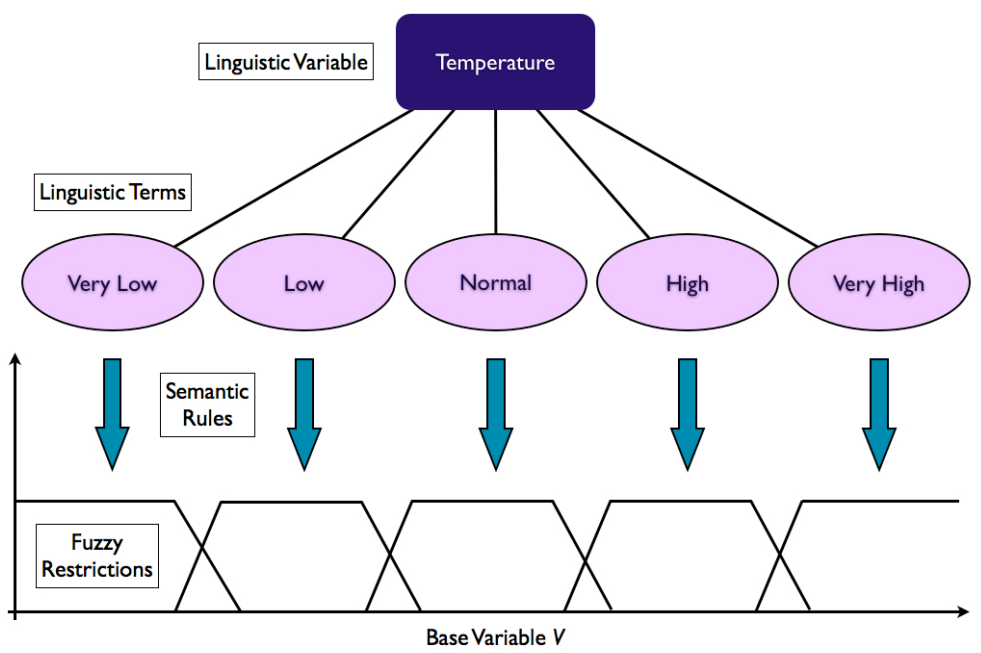

Along with the linguistic variables is frequent the use of linguistic modifiers. A linguistic modifier is a unitary operator $h:[0,1] \rightarrow[0,1]$ applied to a fuzzy set -no sense in a crisp set- to introduce a nuance like "very", "much", "pretty", etc.

The linguistic modifier is usually associated with a function to handle it 
within the fuzzy system. For example, some common associations are:

$$
\begin{gathered}
\text { "Very" } \rightarrow h(a)=a^{2}, \text { where } a \in[0,1] \\
\text { "More or less" } \rightarrow h(a)=\sqrt{a}, \text { where } a \in[0,1]
\end{gathered}
$$

In general, if $h(a)<a$ to the modifier $h$ is applied the adjective of strong modifier. In the opposite case the switch is known as weak modifier.

Modifiers satisfy the following properties:

- $h(0)=0$ and $h(1)=1$

- $h$ is a continuous function

- If $h$ is strong then $h^{-1}$ is weak

- Any composition of two modifiers is a new modifier

A similar concept of linguistic variable is the fuzzy variable. The fuzzy variables take as values elements of fuzzy sets, but do not have associated linguistic terms.

\section{Fuzzy Logic Applied to the Control Systems:}

The first theoretical developments of fuzzy logic were clearly geared to solving the generic problem of control systems. These developments began to be implemented in the mid-70s, when computer technology reached a certain degree of maturity. Professor Zadeh proposed the implementation of control systems based on the same paradigm with which a person acts: usually does not require a completely accurate information, numerical, and yet is able to present a high degree of adaptation in the control tasks. Translating that

principle, if the control systems are developed so that they are prepared to work with inaccurate information will then be much more effective than classical systems. 
In a way it is the same idea in the basis of technological development that underlies the Internet, which begins to take shape in the late 60's, and consists of a communication protocol designed to operate in an imperfect environment, noisy, even with a number of nodes totally or partially damaged. The network elements works under the premise of "best can do": when they receive a packet, analyzes the source and destination addresses and forwarded by the most appropriate interface based on these directions and the network status. Thus, all nodes cooperate to the correct flow of messages, but the action of each alone is very limited and can be replaced by the operation of another.

In this context, fuzzy logic is a control systems methodology that provides an easy way to reach a conclusion based on inaccurate, ambiguous, vague or incomplete information. Fuzzy Logic Control addresses the problems mimicking the way a person solves small problems and make decisions.

Fuzzy Logic incorporates a rule IF X AND Y THEN Z to the problem of control systems. The goal is to be based more on the experience that an operator can have about the performance of a system than on precise knowledge of the design, architecture or operating parameters.

For example, if it comes to controlling the temperature, instead of introducing clauses such $T=80, T>60 T>20$ There are used these other:

IF ("The system is cold") AND ("The system is increasingly cold") THEN ("Increase temperature")

These terms are vague yet perfectly descriptive of what is happening and therefore very suitable for decision making.

\section{Applied Control Systems Fuzzy Logic:}

Control Systems Fuzzy Logic are very robust since they do not require accurate free from interference information and are easily adaptable to stop or malfunction of one or more sensors, in a similar manner as the Internet to 
the fall of one or more nodes.

Allow easy modification of the control rules, since these rules are based on user and machine behavior. For the same reason it is possible to easily add or remove sensors.

They support general purpose and affordable sensors in many cases, since no accurate system specific parameters or precise measurements are required.

Also, they allow more input rules and output responses.

They are suitable for nonlinear systems or systems that can not be modeled mathematically, which opens a door to the control of systems that have not traditionally been used in automation.

But how is applied in practice to fuzzy logic control systems? Here are the basic steps of this implementation:

1. Define the objectives and criteria of the control system in terms of questions like what system we want to control? What should I do to control the system? What kind of answers I need? What are the possible situations of system failure?

2. Identify relationships between inputs and outputs and select the set of fuzzy variables (usually error conditions and degrees of change in the error conditions).

3. Divide the control problem into a series of rules of the type IF X AND Y THEN Z, that define the desired response based on a series of preset input conditions.

4. Create the membership functions of fuzzy logic to define the meaning of input and output terms used in the rules.

These terms are vague yet perfectly descriptive of what is happening and therefore very suitable for decision making. 


\section{Chapter 2}

\section{Background}

\subsection{Fuzzy metric and fuzzy quasi-metric spaces}

In this section we recall several notions and properties from the theory of quasi-metric spaces and fuzzy (quasi-)metric spaces that will be useful later on.

Throughout this work the letters $\mathbb{R}, \mathbb{R}^{+}, \omega$ and $\mathbb{N}$ will denote the set of real numbers, the set of nonnegative real numbers, the set of nonnegative integer numbers and the set of positive integer numbers, respectively.

Our basic references for quasi-metric spaces are [22] and [43].

Following the modern terminology, a quasi-metric space is a pair $(X, d)$ such that $X$ is a set and $d$ is a function from $X \times X$ to $\mathbb{R}^{+}$such that for all $x, y, z \in X:$

(i) $x=y$ if and only if $d(x, y)=d(y, x)=0$;

(ii) $d(x, z) \leq d(x, y)+d(y, z)$.

If $(X, d)$ is a quasi-metric space, we say that $d$ is a quasi-metric on $X$.

Given a quasi-metric $d$ on $X$, the function $d^{-1}$ defined on $X \times X$ by $d^{-1}(x, y)=d(y, x)$, is also a quasi-metric on $X$, called the conjugate of $d$, 
and the function $d^{s}$ defined on $X \times X$ by $d^{s}(x, y)=\max \left\{d(x, y), d^{-1}(x, y)\right\}$ is a metric on $X$.

A quasi-metric space $(X, d)$ is said to be bicomplete if $\left(X, d^{s}\right)$ is a complete metric space. In this case, we say that $d$ is a bicomplete quasi-metric on $X$.

Each quasi-metric $d$ on $X$ induces a $T_{0}$ topology $\tau_{d}$ on $X$ which has as a base the family of open balls $\left\{B_{d}(x, \varepsilon): x \in X, \varepsilon>0\right\}$, where $B_{d}(x, \varepsilon)=\{y \in X: d(x, y)<\varepsilon\}$ for all $x \in X$ and $\varepsilon>0$.

Several authors have investigated the question of obtaining a suitable notion of fuzzy metric spaces and various different definitions have been proposed and discussed.

Here we shall use a slight modification of the notion of fuzzy metric space as defined by Kramosil and Michalek in [42]. This notion, which is closely related with the notion of a Menger space, has the advantage that induces, in a natural way, a metrizable topology in the classical sense.

We need the following notion.

Definition 2.1 ([72, 73]). A binary operation $*:[0,1]^{2} \rightarrow[0,1]$ is called a continuous t-norm if: (i) $*$ is associative and commutative; (ii) $*$ is continuous; (iii) $a * 1=a$ for all $a \in[0,1]$; and (iv) $a * b \leq c * d$ whenever $a \leq c$ and $b \leq d$.

Typical instances of continuous t-norm are $\wedge, \cdot$ and $*_{L}$, where, for all $a, b \in[0,1], a \wedge b=\min \{a, b\}, a \cdot b=a b$, and $*_{L}$ is the Lukasiewicz t-norm defined by $a *_{L} b=\max \{a+b-1,0\}$.

It is easy to check that $*_{L} \leq \leq \wedge$. In fact $* \leq \wedge$ for all continuous t-norms $*$. 
Definition 2.2 (compare [42]). A fuzzy metric space is a triple $(X, M, *)$ such that $*$ is a continuous t-norm and $M$ is a fuzzy set in $X \times X \times \mathbb{R}^{+}$(i.e., $M$ is a function from $X \times X \times \mathbb{R}^{+}$to $\left.[0,1]\right)$ such that for all $x, y, z \in X$ :

(i) $M(x, y, 0)=0$;

(ii) $x=y$ if and only if $M(x, y, t)=1$ for all $t>0$;

(iii) $M(x, y, t)=M(y, x, t)$;

(iv) $M(x, z, t+s) \geq M(x, y, t) * M(y, z, s)$ for all $t, s \geq 0$;

(v) $M\left(x, y,_{-}\right): \mathbb{R}^{+} \rightarrow[0,1]$ is left continuous.

If $(X, M, *)$ is a fuzzy metric space, we say that the pair $(M, *)$ is a fuzzy metric on $X$.

Remark 2.3. The original notion of a fuzzy metric space given by Kramosil and Michalek in [42], includes the condition $\lim _{t \rightarrow+\infty} M(x, y, t)=1$ for all $x, y \in X$. However, this condition will be not necessary in our context.

Remark 2.4. George and Veeramani introduced in [23] (see also [24]) a stronger but interesting notion of fuzzy metric space which will not be discussed in this thesis.

It is well known, and easy to see, that for each $x, y \in X, M\left(x, y,{ }_{-}\right)$is a nondecreasing function on $\mathbb{R}^{+}$.

We also recall that each fuzzy metric $(M, *)$ on a set $X$ induces a topology $\tau_{M}$ on $X$ which has a base the family of open balls $\left\{B_{M}(x, \varepsilon, t): x \in X\right.$, $\varepsilon \in(0,1), t>0\}$, where $B_{M}(x, \varepsilon, t)=\{y \in X: M(x, y, t)>1-\varepsilon\}$.

It immediately follows the useful fact that a sequence $\left(x_{n}\right)_{n}$ in a fuzzy metric space $(X, M, *)$ converges to $x \in X$, with respect to $\tau_{M}$, if and only if $\lim _{n \rightarrow \infty} M\left(x, x_{n}, t\right)=1$ for all $t>0$. 
Definition 2.5 ([24, 72, 73]). Let $(X, M, *)$ be a fuzzy metric space.

a) A sequence $\left(x_{n}\right)_{n}$ in $X$ is said to be a Cauchy sequence if for each $t>0$ and $\varepsilon \in(0,1)$ there exists $n_{0} \in \mathbb{N}$ such that $M\left(x_{n}, x_{m}, t\right)>1-\varepsilon$ for all $n, m \geq n_{0}$.

b) $(X, M, *)$ is said to be complete if every Cauchy sequence converges with respect to $\tau_{M}$.

It is well known (see e.g. [31, 72, 73]) every fuzzy metric space $(X, M, *)$ is metrizable, i.e., there exists a metric $d$ on $X$ whose induced topology coincides with $\tau_{M}$.

The following two well-known examples show that the converse is also true, i.e., every metrizable topological space $(X, \tau)$ admits a fuzzy metric whose induced topology coincides with $\tau$.

Example 2.6. Let $(X, d)$ be a metric space. Define $M_{01}: X \times X \times \mathbb{R}^{+} \rightarrow$ $[0,1]$ by

$$
\begin{aligned}
& M_{01}(x, y, t)=0 \quad \text { if } t \leq d(x, y), \quad \text { and } \\
& M_{01}(x, y, t)=1 \quad \text { if } t>d(x, y) .
\end{aligned}
$$

Then, for each continuous t-norm $*,\left(M_{01}, *\right)$ is a fuzzy metric on $X$ such that its induced topology $\tau_{M_{01}}$ coincides with the topology induced by $d$. Moreover $\left(X, M_{01}, *\right)$ is complete if and only if $(X, d)$ is complete.

Example 2.7. Let $(X, d)$ be a metric space. Define $M_{d}: X \times X \times \mathbb{R}^{+} \rightarrow$ $[0,1]$ by $M_{d}(x, y, 0)=0$ and

$$
M_{d}(x, y, t)=\frac{t}{t+d(x, y)},
$$

for all $t>0$.

Then, for each continuous t-norm $*,\left(M_{d}, *\right)$ is a fuzzy metric on $X$ (in fact, it is a fuzzy metric in the sense of George and Veeramani) called the 
standard fuzzy metric of $(X, d)([23])$. Moreover, the topology $\tau_{M_{d}}$ coincides with the topology induced by $d$, and $\left(X, M_{d}, *\right)$ is complete if and only if $(X, d)$ is complete.

In [32] (see also [14]), Gregori and Romaguera introduced quasi-metric generalizations of the notions of fuzzy metric space from Kramosil and Michalek, and by George and Veeramani, respectively. We shall use the following.

Definition $2.8([14,32])$. A fuzzy quasi-metric space is a triple $(X, M, *)$ such that $*$ is a continuous t-norm and $M$ is a fuzzy set in $X \times X \times \mathbb{R}^{+}$such that for all $x, y, z \in X$ :

(i) $M(x, y, 0)=0$;

(ii) $x=y$ if and only if $M(x, y, t)=M(y, x, t)=1$ for all $t>0$;

(iii) $M(x, z, t+s) \geq M(x, y, t) * M(y, z, s)$ for all $t, s \geq 0$;

(iv) $M\left(x, y,{ }_{-}\right): \mathbb{R}^{+} \rightarrow[0,1]$ is left continuous.

If $(X, M, *)$ is a fuzzy quasi-metric space, we say that the pair $(M, *)$ is a fuzzy quasi-metric on $X$.

As in the fuzzy metric case, if $(M, *)$ is a fuzzy quasi-metric on $X$, then for each $x, y \in X, M\left(x, y,_{-}\right)$is a nondecreasing function on $\mathbb{R}^{+}$.

If $(M, *)$ is a fuzzy quasi-metric on $X$, then $\left(M^{-1}, *\right)$ is also a fuzzy quasi-metric on $X$, where $M^{-1}$ is the fuzzy set in $X \times X \times \mathbb{R}^{+}$defined by $M^{-1}(x, y, t)=M(y, x, t)$. Moreover $\left(M^{i}, *\right)$ is a fuzzy metric on $X$ where $M^{i}$ the fuzzy set in $X \times X \times \mathbb{R}^{+}$defined by $M^{i}(x, y, t)=\min \left\{M(x, y, t), M^{-1}(x, y, t)\right\}$ $([32])$.

A fuzzy quasi-metric space $(X, M, *)$ is said to be bicomplete if the fuzzy metric space $\left(X, M^{i}, *\right)$ is complete. In this case we say that $(M, *)$ is a bicomplete fuzzy quasi-metric on $X$. 
Similarly to the fuzzy metric case (see [14, 32]), each fuzzy quasi-metric $(M, *)$ on a set $X$ induces a topology $\tau_{M}$ on $X$ which has a base the family of open balls $\left\{B_{M}(x, \varepsilon, t): x \in X, \varepsilon \in(0,1), t>0\right\}$, where $B_{M}(x, \varepsilon, t)=$ $\{y \in X: M(x, y, t)>1-\varepsilon\}$.

It immediately follows that a sequence $\left(x_{n}\right)_{n}$ in a fuzzy quasi-metric space $(X, M, *)$ converges to $x \in X$, with respect to $\tau_{M}$, if and only if $\lim _{n \rightarrow \infty} M\left(x, x_{n}, t\right)=1$ for all $t>0$.

Gregori and Romaguera showed in [32] that every fuzzy quasi-metric space $(X, M, *)$ is quasi-metrizable, i.e., there exists a quasi-metric $d$ on $X$ whose induced topology coincides with $\tau_{M}$.

Conversely, if $(X, d)$ is a quasi-metric space, then $\left(M_{01}, *\right)$ and $\left(M_{d}, *\right)$, defined as in Examples 2.6 and 2.7, respectively, are fuzzy quasi-metrics on $X$ whose induced topologies coincide with the topology induced by $d$. Moreover, bicompleteness of $(X, d),\left(M_{01}, *\right)$ and $\left(M_{d}, *\right)$ are equivalent conditions.

We conclude this section by recalling the notion of isometry for fuzzy (quasi-)metric spaces.

Definition 2.9. Let $(X, M, *)$ and $\left(Y, N, *^{\prime}\right)$ be two fuzzy (quasi-)metric spaces. An isometry from $(X, M, *)$ to $\left(Y, N, *^{\prime}\right)$ is a mapping $f: X \rightarrow Y$ such that

$$
N(f(x), f(y), t)=M(x, y, t),
$$

for all $x, y \in X$ and $t>0$.

If $f$ is an isometry from $(X, M, *)$ onto $\left(Y, N, *^{\prime}\right)$, we say that $(X, M, *)$ and $\left(Y, N, *^{\prime}\right)$ are isometric. 


\subsection{Domain Theory}

In this section we collect several notions and properties from Domain Theory that will be useful later on.

Our basic reference for Domain Theory is [26].

Let us recall that a (partial) order on a (nonempty) set $X$ is a binary relation $\leq$ on $X$ such that for all $x, y, z \in X$ :

(i) $x \leq x$ for all $x \in X$ (reflexivity);

(ii) $x \leq y$ and $y \leq x$ imply $x=y$ (antisymmetry);

(iii) $x \leq y$ and $y \leq z$ imply $x \leq z$ (transitivity).

A partially ordered set, or poset for short, is a set $L$ equipped with a partial order $\leq$. It will be denoted in the sequel by $(L, \leq)$.

Example 2.10. It is well known that if $(X, d)$ is a quasi-metric space, then the binary relation $\leq_{d}$ on $X$ given by

$$
x \leq_{d} y \Longleftrightarrow d(x, y)=0
$$

is a partial order on $X$, which is called the partial order induced by $d$, or the order of specialization.

Hence $\left(X, \leq_{d}\right)$ is a poset.

Example 2.11. If $(X, M, *)$ is a fuzzy quasi-metric space, then the binary relation $\leq_{M}$ on $X$ given by

$$
x \leq_{M} y \Longleftrightarrow M(x, y, t)=1 \quad \text { for all } t>0
$$

is a partial order on $X$, which is called the partial order induced by $(M, *)$.

Hence $\left(X, \leq_{M}\right)$ is a poset. 
Definition 2.12. A subset $D$ of a poset $(L, \leq)$ is directed provided that it is nonempty and every finite subset of $D$ has an upper bound in $D$ (equivalently, if for each $a, b \in D$ there is $c \in D$ such that $a \leq c$ and $b \leq c$ ).

A sequence $\left(x_{n}\right)_{n}$ in a poset $(L, \leq)$ is said to be increasing if $x_{n} \leq x_{n+1}$ for all $n$.

Definition 2.13. A poset $(L, \leq)$ is said to be directed complete, and is called a dcpo, if every directed subset of $L$ has a least upper bound.

The least upper bound of a subset $D$ of $(L, \leq)$ is denoted by $\sqcup D$ if it exists. An element $x$ of $L$ is called maximal if condition $x \leq y$ implies $x=y$. The set of all maximal elements of $L$ is denoted by $\operatorname{Max}((L, \leq))$, or simply by $\operatorname{Max}(L)$ if no confusion arises.

Definition 2.14. Given a poset $(L, \leq)$, we say that $x$ is way-below $y$, in symbols $x \ll y$, if for each directed subset $D$ of $L$ for which $\sqcup D$ exists, the relation $y \leq \sqcup D$ implies the existence of some $z \in D$ with $x \leq z$.

Definition 2.15. A poset $(L, \leq)$ is called continuous if it satisfies the axiom of approximation, i.e., for all $x \in L$, the set $\Downarrow x=\{u \in L: u \ll x\}$ is directed and $x=\sqcup(\Downarrow x)$.

Definition 2.16. A continuous poset which is also a dcpo is called a continuous domain or, simply, a domain.

A subset $B$ of a poset $(L, \leq)$ is a basis for $L$ if for each $x \in L$, the set $\Downarrow x_{B}=\{u \in B: u \ll x\}$ is directed and $x=\sqcup\left(\Downarrow x_{B}\right)$.

Recall that a poset has a basis if and only if it is continuous. Therefore, a depo has a basis if and only if it is a domain. 
Definition 2.17. A dcpo having a countable basis is said to be an $\omega$ continuous domain or simply an $\omega$-domain.

The Scott topology $\sigma(L)$ of a dcpo $(L, \leq)$ is constructed as follows (Chapter II in [26]): A subset $U$ of $L$ is open in the Scott topology provided that:

(i) $U=\uparrow U$, where $\uparrow U=\{y \in X: x \leq y$ for some $x \in U\}$;

(ii) for each directed subset $D$ of $L$ such that $\sqcup D \in U$, it follows that $D \cap U \neq \emptyset$.

If $(L, \leq)$ is a domain, then the sets $\Uparrow x, x \in L$, form an open base for the Scott topology, where $\Uparrow x=\{y \in X: x \ll y\}$ (see Proposition II-1.10 of $[26])$.

Furthermore, the Scott topology has a countable base if and only if $(L, \leq)$ is an $\omega$-domain; in this case, if $B$ is a countable basis for $(L, \leq)$, then the sets $\Uparrow x, x \in B$, is a countable base for the Scott topology (Theorem III-4.5 in $[26])$.

In case that $(L, \leq)$ is a continuous poset it is possible to show yet that the sets $\Uparrow x, x \in L$, form an open base for a topology on $L$, which is also called the Scott topology of $(L, \leq)$ and is also denoted by $\sigma(L)$ (see, for instance, p. 58 in [20]).

We conclude this chapter with the notions of a topological model and of a computational model.

Definition 2.18 ([45]). A model for a topological space $(X, \tau)$ is a pair $(L, \phi)$ such that $L$ is a domain and $\phi: X \rightarrow \operatorname{Max}(L)$ is a homeomorphism, where $\operatorname{Max}(L)$ is endowed with the restriction of the Scott topology of $L$. 
Definition 2.19 (compare [66]). A computational (or quantitative) model for a complete metric space $(X, d)$ is a triple $(L, q, \phi)$ such that $L(=(L, \sqsubseteq))$ is a domain, $q$ is a bicomplete quasi-metric on $L$ and $\phi$ is a map from $X$ into $L$ such that:

(i) $\tau_{q}=\sigma(L)$.

(ii) $\phi$ is an isometry from $(X, d)$ into $(L, q)$.

(iii) $\phi(X)=\operatorname{Max}(L)$. 


\section{Chapter 3}

\section{A domain theoretic approach to fuzzy metric spaces based on the standard fuzzy metric}

\subsection{Introduction}

As we indicated above our main interest is to establish connections between the theory of fuzzy metric spaces and domain theory. In fact, we are interested in extending the theory developed by Edalat and Heckmann ([20, 36]) to the fuzzy framework.

Let us recall that a formal ball in a (non-empty) set $X$ is simply a pair $(x, r)$, with $x \in X$ and $r \in \mathbb{R}^{+}$.

The set of formal balls of $X$ is the Cartesian product $X \times \mathbb{R}^{+}$which will be denoted by $\mathbf{B} X$ in the sequel.

Edalat and Heckmann showed in $[20]$ that if $(X, d)$ is a metric space, then the binary relation $\sqsubseteq_{d}$ defined on $\mathbf{B} X$ by

$$
(x, r) \sqsubseteq_{d}(y, s) \Longleftrightarrow d(x, y) \leq r-s,
$$


for all $(x, r),(y, s) \in \mathbf{B} X$, is a partial order.

Moreover, they proved, among other interesting results, that $\left(\mathbf{B} X, \sqsubseteq_{d}\right)$ is a domain if and only if $(X, d)$ is complete [20, Theorem 6 and Corollary 10].

In order to extending the constructions of Edalat and Heckmann to the fuzzy metric setting, two initial procedures seem to be quiet natural.

The first one consists in noting that condition (3.1) can be formulated as $(x, r) \sqsubseteq_{d}(y, s) \Longleftrightarrow y \in \bar{B}_{d}(x, r-s)$,

(where $\bar{B}_{d}(x, 0)=\{x\}$ ), and then to adapt this equivalence to the fuzzy metric context.

The second one consists in noting that condition (3.1) can be formulated in terms of the standard fuzzy metric $\left(M_{d}, \wedge\right)$ as

$$
(x, r) \sqsubseteq_{d}(y, s) \Longleftrightarrow M_{d}(x, y, t) \geq \frac{t}{t+r-s} \quad \text { for all } t>0,
$$

and then take this equivalence as a starting point to define a possible suitable partial order on the set of formal balls of any fuzzy metric space.

In this chapter we study this second approach, whereas the first one will be discussed in Chapter 4.

In fact, we shall show that for any fuzzy metric space of type $(X, M, \wedge)$, the binary relation suggested by (3.2), and denoted by $\sqsubseteq_{M}$, is a partial order on $\mathbf{B} X$. Moreover, we characterize when the poset $\left(\mathbf{B} X, \sqsubseteq_{M}\right)$ is a domain. This will be done by means of a new notion of fuzzy metric completeness that generalizes the usual one.

The results in this chapter are contained in the papers by L.A. Ricarte and S. Romaguera, "The set of formal balls of a complete fuzzy metric space viewed as a continuous domain" published in the Proceedings of the Workshop in Applied Topology WiAT'12 (cf. [56]), and "A domain-theoretic approach to fuzzy metric spaces", accepted for publication in Topology and its Applications (cf. [57]). 


\subsection{The poset $\left(\mathbf{B} X, \sqsubseteq_{M}\right)$ of formal balls of a fuzzy metric space $(X, M, \wedge)$}

We begin this section with the following notion which is suggested by the equivalence (3.2) given above.

Definition 3.1. For a fuzzy metric space $(X, M, *)$ we define a binary relation $\sqsubseteq_{M}$ on the set $\mathbf{B} X$ of formal balls of $X$, by

$$
(x, r) \sqsubseteq_{M}(y, s) \Longleftrightarrow M(x, y, t) \geq \frac{t}{t+r-s} \quad \text { for all } t>0 .
$$

Remark 3.2. Note that if $(x, r) \sqsubseteq_{M}(y, s)$, then $r \geq s$. Indeed, choose $t_{0}>0$ such that $t_{0}+r-s>0$. Then

$$
1 \geq M\left(x, y, t_{0}\right) \geq \frac{t_{0}}{t_{0}+r-s}>0,
$$

so $t_{0}+r-s \geq t_{0}$, and thus $r \geq s$.

Next we show that for $*=\wedge,\left(\mathbf{B} X, \sqsubseteq_{M}\right)$ is a poset, and give an example of a fuzzy metric space $(X, M, \cdot)$ for which $\left(\mathbf{B} X, \sqsubseteq_{M}\right)$ is not a poset.

Proposition 3.3. Let $(X, M, \wedge)$ be a fuzzy metric space. Then $\left(\mathbf{B} X, \sqsubseteq_{M}\right)$ is a poset.

Proof. Let $(x, r),(y, s),(z, u) \in \mathbf{B} X$. Then we have

- Reflexivity: $(x, r) \sqsubseteq_{M}(x, r)$ because $M(x, x, t)=1$ for all $t>0$.

- Antisymmetry: Let $(x, r) \sqsubseteq_{M}(y, s)$ and $(y, s) \sqsubseteq_{M}(x, r)$. Then $(x, r)=$ $(y, s)$, because under the above assumption, $r=s$, by Remark 3.2, and hence $M(x, y, t)=1 \quad$ for all $t>0$ and thus $x=y$. 
- Transitivity: Let $(x, r) \sqsubseteq_{M}(y, s)$ and $(y, s) \sqsubseteq_{M}(z, u)$. Then $(x, r) \sqsubseteq_{M}$ $(z, u)$ because, assuming without loss of generality that $r>s>u$, and putting for each $t>0, v=t /(r-u)$, we obtain

$$
\begin{aligned}
M(x, z, t) & =M(x, z, v(r-u)) \\
& \geq M(x, y, v(r-s)) \wedge M(y, z, v(s-u)) \\
& \geq \frac{v(r-s)}{v(r-s)+r-s} \wedge \frac{v(s-u)}{v(s-u)+s-u} \\
& =\frac{v}{v+1}=\frac{t}{t+r-u}
\end{aligned}
$$

Thus, we have proved that $\sqsubseteq_{M}$ is a partial order on $\mathbf{B} X$.

Remark 3.4. Note that $\operatorname{Max}\left(\left(X, \sqsubseteq_{M}\right)\right)=\{(x, 0): x \in X\}$.

Remark 3.5. It is clear that if $(X, d)$ is a metric space, the partial orders $\sqsubseteq_{d}$ and $\sqsubseteq_{M_{d}}$ coincide.

The following example shows that we cannot guarantee that the binary relation $\sqsubseteq_{M}$ is a partial order when $*$ is the product.

Example 3.6. Let $X=\{a, b, c\}$ and $M: X \times X \times \mathbb{R}^{+} \rightarrow[0,1]$ defined by

$$
\begin{aligned}
& M(x, y, 0)=M(y, x, 0)=0 \quad \text { for all } x, y \in X \\
& M(a, a, t)=M(b, b, t)=M(c, c, t)=1 \quad \text { for all } t>0 \\
& M(a, b, t)=M(b, a, t)=M(b, c, t)=M(c, b, t)=t /(t+1) \quad \text { for all } t>0, \\
& M(a, c, t)=M(c, a, t)=t^{2} /(t+2)^{2} \quad \text { for all } t>0 .
\end{aligned}
$$


It was proved in $[34$, Example 1] that $(X, M, \cdot)$ is a fuzzy metric space.

Now observe that for $r=2, s=1$ and $u=0$, one has

$$
M(a, b, t)=\frac{t}{t+1}=\frac{t}{t+r-s},
$$

and

$$
M(b, c, t)=\frac{t}{t+1}=\frac{t}{t+s-u},
$$

for all $t>0$. So $(a, r) \sqsubseteq_{M}(b, s)$ and $(b, s) \sqsubseteq_{M}(c, u)$. However, for $0<t<1$, we obtain

$$
M(a, c, t)=\frac{t^{2}}{(t+2)^{2}}<\frac{t}{t+r-u} .
$$

Therefore, the binary relation $\sqsubseteq_{M}$ is not transitive, and thus $\left(\mathbf{B} X\right.$, $\left.\sqsubseteq_{M}\right)$ is not a poset.

Remark 3.7. In a first moment one can think that the following alternative definition of $\sqsubseteq_{M}$, also could provide a partial order on the set $\mathbf{B} X$ :

$$
(x, r) \sqsubseteq_{M}(y, s) \Longleftrightarrow M(x, y, t) \geq \frac{t}{t+r-s} \quad \text { for some } t>0,
$$

However, this is not the case as the next example shows.

Example 3.8. Let $(X, d)$ be a metric space, with $|X| \geq 2$, and consider the fuzzy metric $\left(M_{01}, \wedge\right)$ on $X$ constructed in Example 2.6.

Then, for $x \neq y$ and $r=t>d(x, y)$, we have $(x, r) \sqsubseteq_{M_{01}}(y, r)$ and $(y, r) \sqsubseteq_{M_{01}}(x, r)$, so $\sqsubseteq_{01}$ is not antisymmetric.

\subsection{Complete fuzzy metric spaces of type $(X, M, \wedge)$ and the dcpo $\left(\mathbf{B} X, \sqsubseteq_{M}\right)$}

In this section we study the relation between completeness of a fuzzy metric space $(X, M, \wedge)$ and the fact that its poset of formal balls is a dcpo. We 
shall show the contrarily to the classical metric space, while completeness of $(X, M, \wedge)$ implies that $\left(\mathbf{B} X, \sqsubseteq_{M}\right)$ is a dcpo, the converse does not hold, in general.

We first state some auxiliary results that generalize the corresponding ones obtained by Edalat and Heckmann for metric spaces in [20].

Lemma 3.9. Let $(X, M, \wedge)$ be a fuzzy metric space and let $D$ be a directed subset of $\left(\mathbf{B} X, \sqsubseteq_{M}\right)$. Then, there is an ascending sequence in $D$ which has the same upper bounds as D.

Proof. Let

$$
s=\inf \{r:(x, r) \in D\} .
$$

Then, for each $n \in \mathbb{N}$ there is $\left(y_{n}, s_{n}\right) \in D$ such that $s_{n} \leq s+1 / n$.

Put $\left(x_{1}, r_{1}\right)=\left(y_{1}, s_{1}\right)$. As $D$ is directed there is $\left(x_{2}, r_{2}\right) \in D$ such that $\left(x_{1}, r_{1}\right) \sqsubseteq_{M}\left(x_{2}, r_{2}\right)$ and $\left(y_{2}, s_{2}\right) \sqsubseteq_{M}\left(x_{2}, r_{2}\right)$.

Applying the same reasoning successively we obtain that for each $n>1$ there is $\left(x_{n}, r_{n}\right) \in D$ which is an upper bound of $\left(x_{n-1}, r_{n-1}\right)$ and $\left(y_{n}, s_{n}\right)$. Then $\left(\left(x_{n}, r_{n}\right)\right)_{n}$ is an ascending sequence in $D$.

We shall show that any upper bound of $\left(\left(x_{n}, r_{n}\right)\right)_{n}$ is an upper bound of any element of $D$.

Indeed, let $(z, u) \in D$ such that $\left(x_{n}, r_{n}\right) \sqsubseteq_{M}(z, u)$ for all $n \in \mathbb{N}$ and let $(a, v)$ be an arbitrary element of $D$. Since $D$ is directed, for each $n \in \mathbb{N}$ there is $\left(b_{n}, v_{n}\right) \in D$ which is an upper bound of $(a, v)$ and $\left(x_{n}, r_{n}\right)$.

Note that for each $n \in \mathbb{N}, r_{n} \leq s_{n}$, so

$$
r_{n}-v_{n} \leq s_{n}-v_{n} \leq s-v_{n}+\frac{1}{n} \leq \frac{1}{n},
$$

for all $n \in \mathbb{N}$. 
Now given $t>0$, put

$$
t_{n}=\frac{t}{v-u+2 / n}
$$

for all $n \in \mathbb{N}$.

(Note that $t_{n}$ is well-defined because from $\left(x_{n}, r_{n}\right) \sqsubseteq_{M}(z, u)$ it follows that $u \leq r_{n}$, and from $\left(x_{n}, r_{n}\right) \sqsubseteq_{M}\left(b_{n}, v_{n}\right)$ it follows that $v_{n} \leq r_{n} \leq v_{n}+$ $1 / n \leq v+1 / n$. Hence $v-u+1 / n \geq 0$, and thus, $v-u+2 / n>0$, for all $n \in \mathbb{N})$.

Therefore

$$
t_{n}\left(v-u+2 r_{n}-2 v_{n}\right) \leq \frac{t(v-u+2 / n)}{v-u+2 / n}=t
$$

for all $n \in \mathbb{N}$, so

$$
\begin{aligned}
& M(a, z, t) \\
\geq & M\left(a, b_{n}, t_{n}\left(v-v_{n}\right)\right) \wedge M\left(b_{n}, x_{n}, t_{n}\left(r_{n}-v_{n}\right)\right) \wedge M\left(x_{n}, z, t_{n}\left(r_{n}-u\right)\right) \\
\geq & \frac{t_{n}\left(v-v_{n}\right)}{t_{n}\left(v-v_{n}\right)+v-v_{n}} \wedge \frac{t_{n}\left(r_{n}-v_{n}\right)}{t_{n}\left(r_{n}-v_{n}\right)+r_{n}-v_{n}} \wedge \frac{t_{n}\left(r_{n}-u\right)}{t_{n}\left(r_{n}-u\right)+r_{n}-u} \\
= & \frac{t_{n}}{t_{n}+1}=\frac{t}{t+v-u+2 / n},
\end{aligned}
$$

for all $n \in \mathbb{N}$. Hence

$$
M(a, z, t) \geq \frac{t}{t+v-u} .
$$

We conclude that $(z, u)$ is an upper bound of $D$.

Lemma 3.10. Let $(X, M, \wedge)$ be a fuzzy metric space. If $\left(\left(x_{n}, r_{n}\right)\right)_{n}$ is an ascending sequence in $(\mathbf{B} X, \sqsubseteq)$, then $\left(x_{n}\right)_{n}$ is a Cauchy sequence in 
$(X, M, \wedge)$ and $\left(r_{n}\right)_{n}$ is a Cauchy sequence in $\mathbb{R}^{+}$.

Proof. Since the sequence $\left(\left(x_{n}, r_{n}\right)\right)_{n}$ is ascending, $\left(x_{n}, r_{n}\right) \sqsubseteq_{M}\left(x_{n+1}, r_{n+1}\right)$, so $r_{n} \geq r_{n+1}$ for all $n \in \mathbb{N}$.

Hence, there exists $r \in \mathbb{R}^{+}$such that $\lim _{n \rightarrow \infty} r_{n}=r$. So, in particular, $\left(r_{n}\right)_{n}$ is a Cauchy in $\mathbb{R}^{+}$.

Now, let us prove that $\left(x_{n}\right)_{n}$ is a Cauchy sequence in $(X, M, \wedge)$.

Indeed, choose $\varepsilon \in(0,1)$ and $t>0$. It is clear that there is $\delta>0$ such that $t /(t+\delta)>1-\varepsilon$.

Since $\left(r_{n}\right)_{n}$ is a Cauchy sequence, there is $n_{0} \in \mathbb{N}$ such that $0 \leq r_{n}-r_{m}<\delta$ whenever $n_{0} \leq n \leq m$. Hence

$$
\frac{t}{t+r_{n}-r_{m}}>\frac{t}{t+\delta}>1-\varepsilon
$$

for $m \geq n \geq n_{0}$.

Since $\left(x_{n}, r_{n}\right) \sqsubseteq_{M}\left(x_{m}, r_{m}\right)$, it follows that

$$
M\left(x_{n}, x_{m}, t\right) \geq \frac{t}{t+r_{n}-r_{m}}>1-\varepsilon
$$

for $m \geq n \geq n_{0}$.

We conclude that $\left(x_{n}\right)_{n}$ is a Cauchy sequence in $(X, M, \wedge)$.

Lemma 3.11. Let $(X, M, \wedge)$ be a fuzzy metric space. If $\left(\left(x_{n}, r_{n}\right)\right)_{n}$ is an ascending sequence in $\left(\mathbf{B} X, \sqsubseteq_{M}\right)$, with $\lim _{n \rightarrow \infty} x_{n}=x$ and $\lim _{n \rightarrow \infty} r_{n}=r$, then $(x, r)=\sqcup D$, where $D=\left\{\left(x_{n}, r_{n}\right): n \in \mathbb{N}\right\}$.

Proof. We first prove that $(x, r)$ is an upper bound of $D$. Indeed, fix $k \in \mathbb{N}$. We want to show that $\left(x_{k}, r_{k}\right) \sqsubseteq_{M}(x, r)$.

Since $\left(r_{n}\right)_{n}$ is a decreasing sequence, then $r \leq r_{n}$ for all $n \in \mathbb{N}$, so, in particular, $r \leq r_{k}$. 
- If $r=r_{k}$, we deduce that $r=r_{n}$ for all $n \geq k$. Hence, from the fact that $\left(x_{k}, r_{k}\right) \sqsubseteq_{M}\left(x_{n}, r_{n}\right)$ for all $n \geq k$, it follows that

$$
M\left(x_{k}, x_{n}, t\right) \geq \frac{t}{t+r_{k}-r_{n}}=\frac{t}{t+r-r}=1,
$$

for all $n \geq k$ and $t>0$.

Therefore $x_{n}=x_{k}$ for all $n \geq k$, and thus, $x=x_{n}$ for all $n \geq k$.

Consequently $(x, r)$ is an upper bound of $D$.

- If $r<r_{k}$, we have that

$$
\frac{t}{t+r_{k}-r}<1
$$

for all $t>0$. Then, there exists $\varepsilon_{0}>0$ such that

$$
\frac{t}{t+r_{k}-r}<1-\varepsilon_{0}
$$

for all $t>0$.

Now fix $t>0$. For each $\varepsilon \in\left(0, \varepsilon_{0} \wedge t\right)$, there exists $m>k$ such that $M\left(x, x_{m}, \varepsilon\right)>1-\varepsilon$ because $\lim _{n \rightarrow \infty} x_{n}=x$. Hence

$$
\begin{aligned}
M\left(x, x_{k}, t\right) & \geq M\left(x, x_{m}, \varepsilon\right) \wedge M\left(x_{m}, x_{k}, t-\varepsilon\right)> \\
& >(1-\varepsilon) \wedge \frac{t-\varepsilon}{t-\varepsilon+r_{k}-r_{m}} \geq(1-\varepsilon) \wedge \frac{t-\varepsilon}{t-\varepsilon+r_{k}-r} .
\end{aligned}
$$

Taking limits as $\varepsilon \rightarrow 0$, we obtain

$$
M\left(x, x_{k}, t\right) \geq \frac{t}{t+r_{k}-r} .
$$

Since $t>0$ is arbitrary, we conclude that $\left(x_{k}, r_{k}\right) \sqsubseteq_{M}(x, r)$, so $(x, r)$ is an upper bound of $D$.

Finally, suppose that there is $(z, u) \in \mathbf{B} X$ such that $\left(x_{n}, r_{n}\right) \sqsubseteq_{M}(z, u)$ for all $n \in \mathbb{N}$. This implies that $r_{n} \geq u$ for all $n \in \mathbb{N}$, and since $\lim _{n \rightarrow \infty} r_{n}=r$, we have $r \geq u$.

We distinguish two cases, again. 
- If $u=r$, we have $\left(x_{n}, r_{n}\right) \sqsubseteq_{M}(z, r)$ for all $n \in \mathbb{N}$, so

$$
M\left(z, x_{n}, t\right) \geq \frac{t}{t+r_{n}-r},
$$

for all $n \in \mathbb{N}$ and $t>0$. Since $\lim _{n \rightarrow \infty} r_{n}=r$, it follows that, for each $t>0$,

$$
\lim _{n \rightarrow \infty} M\left(z, x_{n}, t\right)=1,
$$

so $z=x$. We have shown that $(x, r)=(z, u)$.

- If $u<r$, we shall suppose that $r<r_{n}$ for all $n \in \mathbb{N}$ (otherwise, there is $k \in \mathbb{N}$ such that $r=r_{n}$ and $x=x_{n}$ for all $n \geq k$, as we proved above, and thus $\left.(x, r) \sqsubseteq_{M}(z, u)\right)$.

Take an arbitrary $t>0$. For each $n \in \mathbb{N}$ put $v_{n}=t /\left(r_{n}-u\right)$. Then

$$
\begin{aligned}
M(x, z, t) & \geq M\left(x, x_{n}, v_{n}\left(r_{n}-r\right)\right) \wedge M\left(x_{n}, z, v_{n}(r-u)\right) \\
& \geq \frac{v_{n}\left(r_{n}-r\right)}{v_{n}\left(r_{n}-r\right)+r_{n}-r} \wedge \frac{v_{n}(r-u)}{v_{n}(r-u)+r-u} \\
& =\frac{v_{n}}{v_{n}+1}=\frac{t}{t+r_{n}-u} .
\end{aligned}
$$

Taking limits as $n \rightarrow \infty$, we obtain

$$
M(x, z, t) \geq \frac{t}{t+r-u} .
$$

Therefore $(x, r) \sqsubseteq_{M}(z, u)$. We conclude that $(x, r)=\sqcup D$.

Proposition 3.12. If $(X, M, \wedge)$ is a complete fuzzy metric space, then $\left(\mathbf{B} X, \sqsubseteq_{M}\right)$ is a dcpo.

Proof. Let $D$ a directed subset of $\left(\mathbf{B} X, \sqsubseteq_{M}\right)$. By Lemma 3.9 we know that there is an ascending sequence $\left(\left(x_{n}, r_{n}\right)\right)_{n}$ in $D$ which has the same upper 
bounds as $D$. By Lemma 3.10, $\left(x_{n}\right)_{n}$ is a Cauchy sequence in $(X, M, \wedge)$ and $\left(r_{n}\right)_{n}$ is a Cauchy sequence in $\mathbb{R}^{+}$, so there exists $r \in \mathbb{R}^{+}$such that $\lim _{n \rightarrow \infty} r_{n}=r$.

Since $(X, M, \wedge)$ is complete, there exists $x \in X$ such that $\lim _{n \rightarrow \infty} x_{n}=x$.

Finally, by Lemma 3.11, $(x, r)=\sqcup E$, where $E=\left\{x_{n}: n \in \mathbb{N}\right\}$, and by Lemma 3.10, $(x, r)=\sqcup D$. We conclude that $\left(\mathbf{B} X, \sqsubseteq_{M}\right)$ is a dcpo.

In the classical metric case, a fundamental result ([20, Theorem 6]) states that a metric space is complete if and only if its poset of formal balls is a dcpo. Although Proposition 3.12 above provides a natural extension to our context of the "only if" part of this result, the next example shows that, nevertheless, there exists a non complete fuzzy metric space $(X, M, \wedge)$ for which $\left(\mathbf{B} X, \sqsubseteq_{M}\right)$ is a depo.

Example 3.13. Let $X=\{1 / n: n \in \mathbb{N}\}$ and let $d$ be the restriction of the usual metric on $\mathbb{R}$ to $X$, i.e., $d(1 / n, 1 / m)=|1 / n-1 / m|$ for all $n, m \in \mathbb{N}$.

Then $(X, d)$ is a non complete metric space.

Consider the fuzzy metric $(M, \wedge)$ on $X$ constructed as in Example 3.8, i.e.,

$$
M(x, y, t)=1 \text { if } d(x, y)<t \text {, and } M(x, y, t)=0 \text { if } d(x, y) \geq t
$$

Then $(X, M, \wedge)$ is a non complete fuzzy metric space. In fact, a sequence in $X$ is a Cauchy sequence in $(X, M, \wedge)$ if and ony if it is a Cauchy sequence in $(X, d)$.

Let $D$ be a directed subset of $\left(\mathbf{B} X, \sqsubseteq_{M}\right)$. By Lemma 3.9, there is an ascending sequence $\left(\left(x_{n}, r_{n}\right)\right)_{n}$ in $D$ which has the same upper bounds as $D$, and by Lemma 3.10, $\left(x_{n}\right)_{n}$ is a Cauchy sequence in $(X, M, \wedge)$ (hence in $(X, d))$, and $\left(r_{n}\right)_{n}$ is a Cauchy sequence in $\mathbb{R}^{+}$. 
Fix $\varepsilon \in(0,1)$. Then, there exists $n_{\varepsilon} \in \mathbb{N}$ such that $d\left(x_{n_{\varepsilon}}, x_{n}\right)<\varepsilon$ and $\left|r_{n_{\varepsilon}}-r_{n}\right|<\varepsilon$ for all $n \geq n_{\varepsilon}$. Since $\left(x_{n_{\varepsilon}}, r_{n_{\varepsilon}}\right) \sqsubseteq_{M}\left(x_{n}, r_{n}\right)$ for all $n \geq n_{\varepsilon}$, it follows

$$
M\left(x_{n_{\varepsilon}}, x_{n}, t\right) \geq \frac{t}{t+r_{n_{\varepsilon}}-r_{n}} \geq \frac{t}{t+\varepsilon},
$$

for all $n \geq n_{\varepsilon}$ and $t>0$. By definition of $M$ we deduce that

$$
M\left(x_{n_{\varepsilon}}, x_{n}, t\right)=1
$$

for all $n \geq n_{\varepsilon}$ and $t>0$. So $x_{n_{\varepsilon}}=x_{n}$ for all $n \geq n_{\varepsilon}$.

Hence $\left(x_{n_{\varepsilon}}, r\right)=\sqcup D$, where $r=\lim _{n \rightarrow \infty} r_{n}$. We conclude that $\left(\mathbf{B} X, \sqsubseteq_{M}\right)$ is a dcpo.

\subsection{Characterizing $\left(\mathbf{B} X, \sqsubseteq_{M}\right)$ as a domain: Stan- dard complete fuzzy metric spaces}

In this section, and motivated by Example 3.13 above, we discuss the problem of obtaining a fuzzy counterpart of the aforementioned theorem of Edalat and Heckmann that a metric space $(X, d)$ is complete if and only if ( $\left.\mathbf{B} X, \sqsubseteq_{d}\right)$ is a domain. To this end, we introduce a new notion of completeness which is suitable to characterize those fuzzy metric spaces $(X, M, \wedge)$ such that $\left(\mathbf{B} X, \sqsubseteq_{M}\right)$ is a domain.

Definition 3.14. A sequence $\left(x_{n}\right)_{n}$ in a fuzzy metric space $(X, M, *)$ is called a standard Cauchy sequence if for each $\varepsilon \in(0,1)$ there exists $n_{0} \in \mathbb{N}$ such that

$$
M\left(x_{n}, x_{m}, t\right)>\frac{t}{t+\varepsilon},
$$

for all $n, m \geq n_{0}$ and $t>0$. 
Definition 3.15. A fuzzy metric space $(X, M, *)$ is called standard complete if every standard Cauchy sequence converges.

It is easy to see that every standard Cauchy sequence in a fuzzy metric space is a Cauchy sequence, and, hence, every complete fuzzy metric space is standard complete.

Although the notion of standard Cauchy sequence certainly yields a strong property, it is not hard to construct fuzzy metric spaces having non-eventually constant standard Cauchy sequences as the following example shows.

Example 3.16. Let $(X, M, \cdot)$ be a stationary fuzzy metric space (i.e., for each $x, y \in X$, the function $t \rightarrow M(x, y, t)$ is constant [29, Definition $2])$, having non-eventually constant Cauchy sequences, and let $\varphi:(0, \infty) \rightarrow$ $(0, \infty)$ be a non-decreasing and continuous function such that $\varphi(t) \geq t$ for all $t>0$.

According to [29, Example 15], $\left(X, M_{\varphi}, \cdot\right)$ is a fuzzy metric space, where $M_{\varphi}(x, y, 0)=0$ for all $x, y \in X$, and

$$
M_{\varphi}(x, y, t)=\frac{M(x, y, t)+\varphi(t)}{1+\varphi(t)},
$$

for all $x, y \in X$ and $t>0$.

Finally, it is routine to check that any Cauchy sequence in $(X, M, \cdot)$ is a standard Cauchy sequence in $\left(X, M_{\varphi}, \cdot\right)$.

The following result, whose easy proof is omitted, provides a significative class of fuzzy metric spaces for which the notions of completeness and standard completeness coincide, and, in addition, justifies the names of "standard Cauchy" and "standard complete", respectively. 
Proposition 3.17. Let $(X, d)$ be a metric space and let $*$ be a continuous t-norm. Then:

(a) A sequence in $\left(X, M_{d}, *\right)$ is standard Cauchy if and only if it is Cauchy.

(b) $\left(X, M_{d}, *\right)$ is standard complete if and only if it is complete.

In order to prove our main result we also need the following lemmas (note that the first one improves Lemma 3.10 above).

Lemma 3.18. Let $(X, M, \wedge)$ be a fuzzy metric space. If $\left(\left(x_{n}, r_{n}\right)\right)_{n}$ is an ascending sequence in $(\mathbf{B} X, \sqsubseteq)$, then $\left(x_{n}\right)_{n}$ is a standard Cauchy sequence in $(X, M, \wedge)$ and $\left(r_{n}\right)_{n}$ is a Cauchy sequence in $\mathbb{R}^{+}$.

Proof. By Lemma 3.10, $\left(r_{n}\right)_{n}$ is a Cauchy sequence in $\mathbb{R}^{+}$.

In order to prove that $\left(x_{n}\right)_{n}$ is a standard Cauchy sequence in $(X, M, \wedge)$ choose an arbitrary $\varepsilon \in(0,1)$. Then, there is $n_{0} \in \mathbb{N}$ such that $0 \leq r_{n}-r_{m}<\varepsilon$ whenever $n_{0} \leq n \leq m$.

Since $\left(x_{n}, r_{n}\right) \sqsubseteq_{M}\left(x_{m}, r_{m}\right)$ we deduce that

$$
M\left(x_{n}, x_{m}, t\right) \geq \frac{t}{t+r_{n}-r_{m}}>\frac{t}{t+\varepsilon},
$$

for $m \geq n \geq n_{0}$ and $t>0$.

Therefore $\left(x_{n}\right)_{n}$ is a standard Cauchy sequence in $(X, M, \wedge)$.

Lemma 3.19. Let $\left(x_{n}\right)_{n}$ be a standard Cauchy sequence in the fuzzy metric space $(X, M, \wedge)$. Then, there is a subsequence $\left(x_{n_{k}}\right)_{k}$ of $\left(x_{n}\right)$ such that $\left(x_{n_{k}}, 2^{-k}\right)_{k}$ is an ascending sequence in $\left(\mathbf{B} X, \sqsubseteq_{M}\right)$.

Proof. Since $\left(x_{n}\right)_{n}$ is standard Cauchy, there is $n_{1} \in \mathbb{N}$ such that

$$
M\left(x_{n_{1}}, x_{n}, t\right) \geq \frac{t}{t+2^{-2}},
$$


for all $n \geq n_{1}$ and $t>0$. Similarly, there is $n_{2}>n_{1}$ such that

$$
M\left(x_{n_{2}}, x_{n}, t\right) \geq \frac{t}{t+2^{-3}},
$$

for all $n \geq n_{2}$ and $t>0$. Continuing this process, we construct a subsequence $\left(x_{n_{k}}\right)_{k}$ of $\left(x_{n}\right)$ such that

$$
M\left(x_{n_{k}}, x_{n_{k+1}}, t\right) \geq \frac{t}{t+2^{-(k+1)}},
$$

for all $k \in \mathbb{N}$ and $t>0$.

Therefore $\left(x_{n_{k}}, 2^{-k}\right) \sqsubseteq_{M}\left(x_{n_{k+1}}, 2^{-(k+1)}\right)$ for all $k \in \mathbb{N}$. This concludes the proof.

Lemma 3.20. Let $(X, M, \wedge)$ be a standard complete fuzzy metric space. Then $(x, r+\varepsilon) \ll(x, r)$ for all $(x, r) \in \mathbf{B} X$ and for all $\varepsilon>0$.

Proof. Let $D$ be a directed subset of $\mathbf{B} X$ such that there is $(z, u)=\sqcup D$ with $(x, r) \sqsubseteq_{M}(z, u)$.

By Lemma 3.9, there exists an ascending sequence $\left(\left(z_{n}, u_{n}\right)\right)_{n}$ in $D$ for which $(z, u)$ is its least upper bound.

Given $\varepsilon>0$ we shall show that $(x, r+\varepsilon) \sqsubseteq_{M}\left(z_{k}, u_{k}\right)$ for some $k \in \mathbb{N}$.

Indeed, by Lemma $3.18,\left(z_{n}\right)_{n}$ is a standard Cauchy sequence in $(X, M, \wedge)$ and $\left(r_{n}\right)_{n}$ is a Cauchy sequence in $[0, \infty)$, so there is $(y, v) \in \mathbf{B} X$ such that $\lim _{n \rightarrow \infty} z_{n}=y$ and $\lim _{n \rightarrow \infty} u_{n}=v$. By Lemma 3.11, $y=z$ and $v=u$.

Now take $k \in \mathbb{N}$ such that $u_{k}<u+\varepsilon / 2$. We distinguish two cases.

- $r=u$. Then $M(x, z, t)=1$ for all $t>0$ because $(x, r) \sqsubseteq_{M}(z, u)$, so $x=z$. Hence $(x, r)$ is the least upper bound of $\left(\left(z_{n}, u\right)\right)_{n}$, and, in particular,

$$
M\left(x, z_{k}, t\right) \geq \frac{t}{t+u_{k}-r}
$$


for all $t>0$, so

$$
M\left(x, z_{k}, t\right)>\frac{t}{t+(r+\varepsilon)-u_{k}},
$$

for all $t>0$, i.e., $(x, r+\varepsilon) \sqsubseteq_{M}\left(z_{k}, u_{k}\right)$.

- $r>u$. In this case, we have for each $t>0$,

$$
\begin{aligned}
M\left(x, z_{k}, t\right) \geq & M\left(x, z, \frac{r-u}{r+\varepsilon-u_{k}} t\right) \wedge M\left(z, z_{k},\left(1-\frac{r-u}{r+\varepsilon-u_{k}}\right) t\right) \\
\geq & \frac{\left(\frac{r-u}{r+\varepsilon-u_{k}}\right) t}{\left(\frac{r-u}{r+\varepsilon-u_{k}}\right) t+r-u} \wedge \frac{\left(1-\frac{r-u}{r+\varepsilon-u_{k}}\right) t}{\left(1-\frac{r-u}{r+\varepsilon-u_{k}}\right) t+u_{k}-u} \\
= & \frac{(r-u) t}{(r-u) t+(r-u)\left(r+\varepsilon-u_{k}\right)} \\
& \wedge \frac{\left(\varepsilon-u_{k}+u\right) t}{\left(\varepsilon-u_{k}+u\right) t+\left(u_{k}-u\right)\left(r+\varepsilon-u_{k}\right)} \\
= & \frac{t}{t+r+\varepsilon-u_{k}} \wedge \frac{t+\left(\frac{u_{k}-u}{\varepsilon-\left(u_{k}-u\right)}\right)\left(r+\varepsilon-u_{k}\right)}{t} .
\end{aligned}
$$

Since

$$
\frac{u_{k}-u}{\varepsilon-\left(u_{k}-u\right)}=\frac{1}{\frac{\varepsilon}{u_{k}-u}-1}<1
$$

it follows that

$$
\frac{t}{t+r+\varepsilon-u_{k}} \wedge \frac{t}{t+\left(\frac{u_{k}-u}{\varepsilon-\left(u_{k}-u\right)}\right)\left(r+\varepsilon-u_{k}\right)}=\frac{t}{t+r+\varepsilon-u_{k}}
$$

SO

$$
M\left(x, z_{k}, t\right) \geq \frac{t}{t+r+\varepsilon-u_{k}},
$$


for all $t>0$. We conclude that $(x, r+\varepsilon) \ll(x, r)$.

Lemma 3.21. Let $(X, M, \wedge)$ be a fuzzy metric space. If $(x, r) \ll(y, s)$, then there is $\varepsilon \in(0,1)$ such that

$$
M(x, y, t)>\frac{t}{t+r-(s+\varepsilon)} .
$$

So, in particular, $r>s$.

Proof. Take the ascending sequence $(y, s+1 / n)_{n}$.

Since $\lim _{n \rightarrow \infty}(s+1 / n)=s$ and $\{y\}$ may be seen as a constant sequence, by Lemma 3.11 we deduce that $(y, s)$ is the least upper bound of the sequence $((y, s+1 / n))_{n}$.

Since, by hypothesis, $(x, r) \ll(y, s)$, then there exists $n_{0} \in \mathbb{N}$ such that $(x, r) \sqsubseteq_{M}\left(y, s+1 / n_{0}\right)$, i.e.,

$$
M(x, y, t) \geq \frac{t}{t+r-\left(s+1 / n_{0}\right)},
$$

for all $t>0$. Taking $\varepsilon \in\left(0,1 / n_{0}\right)$, we deduce that

$$
M(x, y, t)>\frac{t}{t+r-(s+\varepsilon)},
$$

and, thus, $r>s+\varepsilon$.

Theorem 3.22. For a fuzzy metric space $(X, M, \wedge)$ the following conditions are equivalent.

(1) $(X, M, \wedge)$ is standard complete.

(2) $\left(\mathbf{B} X, \sqsubseteq_{M}\right)$ is a domain.

(3) $\left(\mathbf{B} X, \sqsubseteq_{M}\right)$ is a dcpo.

Proof. $(1) \Longrightarrow(2)$ We first show that $\left(\mathbf{B} X, \sqsubseteq_{M}\right)$ is a depo. 
Let $D$ be a directed subset of $\left(\mathbf{B} X, \sqsubseteq_{M}\right)$. By Lemma 3.9, there is an ascending sequence $\left(\left(x_{n}, r_{n}\right)\right)_{n}$ in $D$ which has the same upper bounds as $D$, and by Lemma 3.18, $\left(x_{n}\right)_{n}$ is a standard Cauchy sequence in $(X, M, \wedge)$ and $\left(r_{n}\right)_{n}$ is a Cauchy sequence in $\mathbb{R}^{+}$.

Since $(X, M, \wedge)$ is standard complete, there exists $x \in X$ such that $\lim _{n \rightarrow \infty} x_{n}=x$. Then, by Lemma 3.11, $(x, r)=\sqcup D$, where $r=\lim _{n \rightarrow \infty} r_{n}$. Hence $\left(\mathbf{B} X, \sqsubseteq_{M}\right)$ is a dcpo.

In order to show that $\left(\mathbf{B} X, \sqsubseteq_{M}\right)$ is continuous take $(x, r),(y, s),(z, u) \in$ $\mathbf{B} X$ such that $(y, s) \ll(x, r)$ and $(z, u) \ll(x, r)$. By Lemma 3.21, there is $\varepsilon \in(0,1)$ such that

$$
M(x, y, t)>\frac{t}{t+s-(r+\varepsilon)} \quad \text { and } \quad M(x, z, t)>\frac{t}{t+u-(r+\varepsilon)},
$$

for all $t>0$. Hence $(y, s) \sqsubseteq_{M}(x, r+\varepsilon)$, and $(z, u) \sqsubseteq_{M}(x, r+\varepsilon)$.

Since, by Lemma 3.20, $(x, r+\varepsilon) \in \Downarrow(x, r)$, we deduce that $\Downarrow(x, r)$ is directed. Moreover $(x, r)$ is, obviously, an upper bound of $\Downarrow(x, r)$.

Finally, let $(z, u) \in \mathbf{B} X$ be such that $(y, s) \sqsubseteq_{M}(z, u)$ for all $(y, s) \in \Downarrow$ $(x, r)$. In particular $(x, r+1 / n) \sqsubseteq_{M}(z, u)$ for all $n \in \mathbb{N}$ by Lemma 3.20, so for each $t>0$ we have

whenever $n \in \mathbb{N}$.

$$
M(x, z, t) \geq \frac{t}{t+r+\frac{1}{n}-u}
$$

Consequently $M(x, z, t) \geq t /(t+r-u)$, i.e., $(x, r) \sqsubseteq_{M}(z, u)$. We conclude that $(x, r)=\sqcup(\Downarrow(x, r))$, and hence $\left(\mathbf{B} X, \sqsubseteq_{M}\right)$ is continuous.

$(2) \Longrightarrow(3)$ Obvious.

$(3) \Longrightarrow(1)$ Let $\left(x_{n}\right)_{n}$ be a standard Cauchy sequence in $(X, M, \wedge)$. By Lemma 3.19, there is a subsequence $\left(x_{n_{k}}\right)_{k}$ of $\left(x_{n}\right)_{n}$ such that $\left(x_{n_{k}}, 2^{-k}\right)_{k}$ is an ascending sequence in $\left(\mathbf{B} X, \sqsubseteq_{M}\right)$. 
Then, there is $(x, r) \in \mathbf{B} X$ such that $(x, r)=\sqcup D$, where $D=\left\{\left(x_{n_{k}}, 2^{-k}\right)\right.$ : $k \in \mathbb{N}\}$. Clearly $r=0$, so $\left(x_{n_{k}}, 2^{-k}\right) \sqsubseteq_{M}(x, 0)$ for all $k \in \mathbb{N}$, and consequently

$$
M\left(x, x_{n_{k}}, t\right) \geq \frac{t}{t+2^{-k}},
$$

for all $k \in \mathbb{N}$ and $t>0$, which implies $\lim _{k \rightarrow \infty} M\left(x, x_{n_{k}}, t\right)=1$ for all $t>0$, i.e., $\lim _{k \rightarrow \infty} x_{n_{k}}=x$.

Since $\left(x_{n}\right)_{n}$ is standard Cauchy, it is, in particular, a Cauchy sequence and hence, $\lim _{n \rightarrow \infty} x_{n}=x$.

We conclude that $(X, M, \wedge)$ is standard complete.

Corollary $3.23([20])$. For a metric space $(X, d)$ the following conditions are equivalent.

(1) $(X, d)$ is complete.

(2) $\left(\mathbf{B} X, \sqsubseteq_{d}\right)$ is a domain.

(3) $\left(\mathbf{B} X, \sqsubseteq_{d}\right)$ is a dcpo.

Proof. (1) $\Longrightarrow(2)$ By Proposition 3.17, $\left(X, M_{d}, \wedge\right)$ is standard complete, so $\left(\mathbf{B} X, \sqsubseteq_{M_{d}}\right)$ is a domain by Theorem 3.22. Now the conclusion follows from Remark 3.5.

$(2) \Longrightarrow(3)$ Obvious.

$(3) \Longrightarrow(1)$ By Remark 3.5, $\left(\mathbf{B} X, \sqsubseteq_{d}\right)$ is a dcpo, so $\left(X, M_{d}, \wedge\right)$ is standard complete by Theorem 3.22. The conclusion follows from Proposition 3.17 .

Remark 3.24. Note that Theorem 3.22 generalizes and improves Proposition 3.12 . 


\subsection{A notion of computational model for com- plete fuzzy metric spaces}

By analogy with the notion of a computational model for a complete metric space as given in Definition 2.19, we propose the following

Definition 3.25. A computational (or quantitative) model for a complete fuzzy metric space $(X, M, *)$ is a triple $(L, Q, \phi)$ such that $L(=(L, \sqsubseteq))$ is a domain, $Q\left(=\left(Q, *^{\prime}\right)\right)$ is a bicomplete fuzzy quasi-metric on $L$ and $\phi$ is a map from $X$ into $L$ such that:

(i) $\tau_{Q}=\sigma(L)$.

(ii) $\phi$ is an isometry from $(X, M, *)$ into $\left(L, Q, *^{\prime}\right)$.

(iii) $\phi(X)=\operatorname{Max}(L)$.

Question 3.26. Is $\left(\mathbf{B} X, \sqsubseteq_{M}\right)$ a computational model for the complete fuzzy metric space $(X, M, \wedge)$ ? 


\section{Chapter 4}

\section{A computational model for complete fuzzy metric spaces}

\subsection{Introduction}

In this chapter we study connections between the theory of fuzzy metric spaces and Domain Theory via the first procedure suggested in Section 3.1. We shall show that the proposed binary relation $\sqsubseteq$ on the set of formal balls $\mathbf{B} X$ of a fuzzy metric space $(X, M, *)$ satisfying $* \geq *_{L}$, is a partial order on $\mathbf{B} X$. In fact, it provides a structure of continuous poset for $\mathbf{B} X$. We also show that $(X, M, *)$ is complete if and only if $(\mathbf{B} X, \sqsubseteq)$ is a domain. Finally, we prove that if $(X, M, *)$ is complete then it admits a computational model.

We shall use the following concepts for a fuzzy metric space $(X, M, *)$.

For each $x \in X, \varepsilon \in[0,1]$ and $t>0$ we define the closed ball $\bar{B}_{M}(x, \varepsilon, t)$ of center $x$ and radius $\varepsilon$ and $t$, as

$$
\bar{B}_{M}(x, \varepsilon, t)=\{y \in X: M(x, y, t) \geq 1-\varepsilon\} .
$$

Note that, in particular, we have $\bar{B}_{M}(x, 1, t)=X$ for all $t>0$.

The main results in this chapter are contained in the paper by L.A. Ricarte and S. Romaguera, "A quantitative computational model for complete 
fuzzy metric spaces", submitted for possible publication (cf. [60]) (a preliminary version of this work was presented by the authors at the 2012 Iberoamerican Conference on Topology and Applications, Guanajuato, Mexico, April 2012).

\subsection{The poset $(\mathrm{B} X, \sqsubseteq)$ of formal balls of a fuzzy metric space $(X, M, *)$ with $* \geq *_{L}$}

Our approach is motivated (see Section 3.1) by the simple observation that the relation

$$
(x, y) \sqsubseteq_{d}(y, s) \Longleftrightarrow d(x, y) \leq r-s,
$$

can be written as

$$
(x, y) \sqsubseteq_{d}(y, s) \Longleftrightarrow y \in \bar{B}_{d}(x, r-s) .
$$

In the light of this fact one can conjecture that the following relation could provide a suitable structure of poset on the set of formal balls of a fuzzy metric space $(X, M, *)$ :

$$
(x, r) \sqsubseteq(y, s) \Longleftrightarrow y \in \bar{B}_{M}(x,(r-s) \wedge 1, r-s) .
$$

However, this relation has some disadvantages. The first one is that $r-s$ should be greater than 0 , so the important case that $r=s$ is excluded. Although we could try to solve this problem by defining $\bar{B}_{M}(x, \varepsilon, 0)=\{x\}$ for all $\varepsilon \in[0,1)$, a second and more important hurdle occurs due to the impossibility of recuperating the theory developed for metric spaces from the partial order $\sqsubseteq_{d}$ by using the relation (4.1). The next easy two examples illustrate this situation.

Example A) Let $X=\{0,2\}$ and let $d$ be the restriction of the usual metric on $\mathbb{R}$ to $X$. Let $\left(M_{d}, \wedge\right)$ be the standard fuzzy metric on $X$. Note 
that $(x, r) \sqsubseteq(y, s)$ for $x=0, y=2, r=1, s=0$ because, in this case, $(r-s) \wedge 1=(1-0) \wedge 1=1$, and thus

$$
M_{d}(x, y, r-s) \geq 0=1-((r-s) \wedge 1) .
$$

However $d(x, y)>r-s$.

Example B) Let $X=\{0,1 / 2\}$ and let $d$ be the restriction of the usual metric on $\mathbb{R}$ to $X$. Let $(M, \wedge)$ be the fuzzy metric on $X$ defined by $M(x, y, t)=$ 0 if $d(x, y) \geq t$ and $M(x, y, t)=1$ if $d(x, y)<t$. Note that $(x, r) \sqsubseteq_{d}(y, s)$ for $x=0, y=1 / 2, r=1 / 2, s=0$.

However $M(x, y, r-s)=0<1 / 2=1-((r-s) \wedge 1)$.

To avoid, at least in part, the above difficulties (see Proposition 4.3 below) we shall consider in the rest of the chapter the following alternative definition for the relation $\sqsubseteq$ on $\mathbf{B} X$ :

$$
(x, r) \sqsubseteq(y, s) \Longleftrightarrow y \in \bigcap_{t>r-s} \bar{B}_{M}(x,(r-s) \wedge 1, t) .
$$

The following equivalence is easily seen and will be used in the sequel.

$$
(x, r) \sqsubseteq(y, s) \Longleftrightarrow \inf _{t>r-s} M(x, y, t) \geq 1-((r-s) \wedge 1) .
$$

Remark 4.1. From the definition of $\sqsubseteq$ we immediately deduce the following easy but useful facts:

a) $(x, r) \sqsubseteq(y, s) \Rightarrow r \geq s$.

b) $(x, r) \sqsubseteq(y, r) \Leftrightarrow x=y$.

c) $r-s \geq 1 \Rightarrow(x, r) \sqsubseteq(y, s)$.

Proposition 4.2. Let $(X, M, *)$ be a fuzzy metric space with $* \geq *_{L}$. Then $(\mathbf{B} X, \sqsubseteq)$ is a poset.

Proof. Let $(x, r),(y, s),(z, u) \in \mathbf{B} X$. Then we have 
- Reflexivity: By Remark $4.1 \mathrm{~b}),(x, r) \sqsubseteq(x, r)$ for all $x \in X$ and $r \in \mathbb{R}^{+}$.

- Antisymmetry: Let $(x, r) \sqsubseteq(y, s)$ and $(y, s) \sqsubseteq(x, r)$. Then, by Remark $4.1 \mathrm{a}), r=s$, and thus

$$
\inf _{t>0} M(x, y, t) \geq 1
$$

i.e., $M(x, y, t)=1$ for all $t>0$, so $x=y$.

- Transitivity: Let $(x, r) \sqsubseteq(y, s)$ and $(y, s) \sqsubseteq(z, u)$. By Remark 4.1 a), $r \geq s \geq u$.

Let $t>r-u$. Choose $\varepsilon \in(0,1)$ such that $t>r-u+2 \varepsilon$. Then

$$
\begin{aligned}
M(x, z, t) & \geq M(x, y, r-s+\varepsilon) * M(y, z, s-u+\varepsilon) \\
& \geq \inf _{t^{\prime}>r-s} M\left(x, y, t^{\prime}\right) * \inf _{t^{\prime}>s-u} M\left(y, z, t^{\prime}\right) \\
& \geq(1-((r-s) \wedge 1)) *(1-((s-u) \wedge 1)) \\
& \geq(1-((r-s) \wedge 1)) *_{L}(1-((s-u) \wedge 1)) \\
& \geq 1-((r-u) \wedge 1) .
\end{aligned}
$$

Hence

$$
\inf _{t>r-u} M(x, z, t) \geq 1-((r-u) \wedge 1) .
$$

We conclude that $(x, r) \sqsubseteq(z, u)$. The proof is complete.

Observe that $\operatorname{Max}((\mathbf{B} X, \sqsubseteq))=\{(x, 0): x \in X\}$.

Although for a given metric space $(X, d)$, the partial order $\sqsubseteq_{d}$ and the partial order $\sqsubseteq$ induced by the standard fuzzy metric $\left(M_{d}, \wedge\right)$ are not related 
in general as Example A above shows, it is possible to prove the following.

Proposition 4.3. Let $(X, d)$ be a metric space with $d \leq 1$, and let $\left(M_{01}, *\right)$ with $* \geq *_{L}$, be the fuzzy metric on $X$ constructed in Example 2.6.

Then $\sqsubseteq_{d}=\sqsubseteq$ on $\mathbf{B} X$.

Proof. Suppose that $(x, r) \sqsubseteq_{d}(y, s)$. Then $d(x, y) \leq r-s$, so for each $t>r-s, M_{01}(x, y, t)=1$, and hence

$$
\inf _{t>r-s} M_{01}(x, y, t)=1
$$

i.e., $(x, r) \sqsubseteq(y, s)$.

Conversely, suppose that $(x, r) \sqsubseteq(y, s)$. If there exists $t_{0}>r-s$ such that $M_{01}\left(x, y, t_{0}\right)=0$, we deduce that $0 \geq 1-((r-s) \wedge 1)$, so $r-s \geq 1$, and thus $1<t_{0} \leq d(x, y)$, a contradiction. Consequently $(x, r) \sqsubseteq_{d}(y, s)$.

The following slight modification of Example A shows that condition $d \leq 1$ cannot be omitted in Proposition 4.3.

Let $X=\{0,2\}$ and let $d$ be the restriction of the usual metric on $\mathbb{R}$ to $X$. Let $(M, \wedge)$ defined as in Proposition 4.3. Then $(x, r) \sqsubseteq(y, s)$ for $x=0$, $y=2, r=1$, and $s=0$, because, in this case, $(r-s) \wedge 1=(1-0) \wedge 1=1$, and thus

$$
M(x, y, r-s) \geq 0=1-((r-s) \wedge 1)
$$

However $d(x, y)>r-s$.

Next we give an example which shows that Proposition 4.2 cannot be generalized for every continuous t-norm. 
Example 4.4. Denote by $*_{Y}$ the continuous t-norm of the Yager class given by

$$
a *_{Y} b=1-\min \left\{1,(\sqrt{1-a}+\sqrt{1-b})^{2}\right\}
$$

for all $a, b \in[0,1]$.

It is well known that $*_{Y} \lesseqgtr *_{L}$.

Now let $X=\{0,1,2\}$ and let $M: X \times X \times \mathbb{R}^{+} \rightarrow[0,1]$ defined as

$$
\begin{aligned}
& M(x, y, 0)=0 \quad \text { for all } x, y \in X \\
& M(x, x, t)=1 \quad \text { for all } x \in X \text { and } t>0 ; \\
& M(0,2, t)=M(2,0, t)=1 / 4 \text { for all } t>0, \text { and } \\
& M(x, y, t)=3 / 4 \quad \text { otherwise. }
\end{aligned}
$$

It is easy to check that $\left(M, *_{Y}\right)$ is a fuzzy metric on $X$ (note, in particular, that $\left.(3 / 4) *_{Y}(3 / 4)=0\right)$.

We show that for the metric space $\left(X, M, *_{Y}\right)$, $\sqsubseteq$ is not a partial order on $\mathbf{B} X$ because it is not transitive. To this end, first note that $(0,1 / 2) \sqsubseteq(1,1 / 4)$ and $(1,1 / 4) \sqsubseteq(2,0)$. In fact

$$
\inf _{t>\left(\frac{1}{2}-\frac{1}{4}\right)} M(0,1, t)=\frac{3}{4}=1-\left(\left(\frac{1}{2}-\frac{1}{4}\right) \wedge 1\right),
$$

and

$$
\inf _{t>\left(\frac{1}{4}-0\right)} M(1,2, t)=\frac{3}{4}=1-\left(\left(\frac{1}{4}-0\right) \wedge 1\right) .
$$

However

$$
\inf _{t>\left(\frac{1}{2}-0\right)} M(0,2, t)=\frac{1}{4}<1-\left(\left(\frac{1}{2}-0\right) \wedge 1\right)
$$

We conclude this section by watching that the partial order $\sqsubseteq_{M}$ defined in Chapter 3 and the partial order $\sqsubseteq$ defined in this chapter are not related in general. 
Indeed, note that in Example A) for $x=0, y=2, r=1, s=0$, one has

$$
\begin{aligned}
\inf _{t>r-s} M_{d}(x, y, t) & =M_{d}(x, y, r-s)=\frac{r-s}{r-s+d(x, y)} \\
& =\frac{1}{3}>1-((r-s) \wedge 1),
\end{aligned}
$$

so $(x, r) \sqsubseteq(y, s)$.

However, the relation $(x, r) \sqsubseteq_{M_{d}}(y, s)$ does not hold by Remark 3.5 because $d(x, y)>r-s$.

Now, consider the fuzzy metric space $(X, M, \wedge)$ where $X=\{0,1\}$ and $(M, \wedge)$ is the fuzzy metric on $X$ defined by $M(x, y, 0)=0$ for all $x, y \in X$, $M(x, x, t)=1$ for all $x \in X$ and $t>0$, and

$$
M(x, y, t)=\frac{t}{t+\frac{1}{3}},
$$

otherwise.

Take $x=0, y=1, r=1 / 3$ and $s=0$. Then

$$
M(x, y, t)=\frac{t}{t+\frac{1}{3}}=\frac{t}{t+r-s},
$$

for all $t>0$, so $(x, r) \sqsubseteq_{M}(y, s)$.

However, the relation $(x, r) \sqsubseteq(y, s)$ does not hold because

$$
\inf _{t>r-s} M(x, y, t)=\frac{1}{2}<\frac{2}{3}=1-((r-s) \wedge 1)
$$

\subsection{Relations between fuzzy metric spaces and properties of Domain Theory via the poset $(\mathbf{B} X, \sqsubseteq)$}

In this section we shall obtain a crucial result that allows us to deduce several nice relations between (complete) fuzzy metric spaces and relevant properties 
of Domain Theory from the corresponding ones for (complete) metric spaces obtained by Edalat and Heckmann in [20].

Recall (see e.g. $[49,54])$ that given a fuzzy metric space $(X, M, *)$ with $* \geq *_{L}$, then the function $d(M)$ defined by

$$
d(M)(x, y)=\sup \{t \geq 0: M(x, y, t) \leq 1-t\}
$$

is a metric on $X$, with $d(M) \leq 1$, whose induced topology coincides with the topology induced by $(M, *)$. In fact, for each $x, y \in X$ and each $\varepsilon \in(0,1)$ we have

$$
M(x, y, \varepsilon)>1-\varepsilon \Longleftrightarrow d(M)(x, y)<\varepsilon
$$

and, as a consequence, the following two facts which will be useful latter on.

Proposition 4.5. Let $\left(x_{n}\right)_{n}$ be a sequence in a fuzzy metric space $(X, M, *)$ where $* \geq *_{L}$. Then $\left(x_{n}\right)_{n}$ is a Cauchy sequence in $(X, M, *)$ if and only if it is a Cauchy sequence in $(X, d(M))$.

Corollary 4.6. Let $(X, M, *)$ be a fuzzy metric space with $* \geq *_{L}$. Then $(X, M, *)$ is complete if and only if $(X, d(M))$ is complete.

The next result gives us the precise relationship between the partial order $\sqsubseteq$ of the fuzzy metric space $(X, M, *)$, with $* \geq *_{L}$ and the partial order $\sqsubseteq_{d(M)}$ of Edalat and Heckmann on the formal balls of the metric space $(X, d(M))$.

Proposition 4.7. Let $(X, M, *)$ be a fuzzy metric space with $* \geq *_{L}$. Then:

$$
(x, r) \sqsubseteq(y, s) \Leftrightarrow(x, r) \sqsubseteq_{d(M)}(y, s), \text { for all }(x, r),(y, s) \in \mathbf{B} X, \text { i.e. },
$$
$\sqsubseteq=\sqsubseteq d(M)$ on $\mathbf{B} X$. 
Proof. Suppose that $(x, r) \sqsubseteq(y, s)$. Then, for each $t>r-s$, we have

$$
M(x, y, t) \geq 1-((r-s) \wedge 1)>1-t .
$$

Hence, $t \leq r-s$ whenever $M(x, y, t) \leq 1-t$, and thus $d(M)(x, y) \leq r-s$, i.e., $(x, r) \sqsubseteq d(M)(y, s)$.

Conversely, suppose that $(x, r) \sqsubseteq_{d(M)}(y, s)$.

If $r-s \geq 1$, then $(x, r) \sqsubseteq(y, s)$ by Remark $4.1 \mathrm{c})$.

If $r-s \in[0,1)$, put $\alpha=\inf _{t>r-s} M(x, y, t)$. Choose an arbitrary $\delta \in$ $(0,1)$. Then, there is $t_{0}>r-s$ such that $\alpha+\delta>M\left(x, y, t_{0}\right)$. Then for $t_{1} \in(r-s, r-s+\delta)$ with $t_{1} \leq t_{0}$, we have the following relations:

$$
\alpha+\delta>M\left(x, y, t_{0}\right) \geq M\left(x, y, t_{1}\right)>1-t_{1}>1-(r-s+\delta),
$$

and thus $2 \delta+\alpha>1-(r-s)$. We conclude that

$$
\inf _{t>r-s} M(x, y, t) \geq 1-(r-s)=1-((r-s) \wedge 1)
$$

i.e., $(x, r) \sqsubseteq(y, s)$.

The main result of this section (Theorem 4.9) is now a direct consequence of the preceding proposition and the next one that collects several results by Edalat and Heckmann.

Proposition $4.8([20])$. Let $(X, d)$ be a metric space. Then, the following hold:

(1) $\left(\mathbf{B} X, \sqsubseteq_{d}\right)$ is a continuous poset.

(2) $(X, d)$ is complete if and only if $\left(\mathbf{B} X, \sqsubseteq_{d}\right)$ is a domain.

(3) $(X, d)$ is complete and separable if and only if $\left(\mathbf{B} X, \sqsubseteq_{d}\right)$ is an $\omega$ domain. 
Theorem 4.9. Let $(X, M, *)$ be a fuzzy metric space with $* \geq *_{L}$. Then, the following hold:

(1) $(\mathbf{B} X, \sqsubseteq)$ is a continuous poset.

(2) $(X, M, *)$ is complete if and only if $(\mathbf{B} X, \sqsubseteq)$ is a domain.

(3) $(X, M, *)$ is complete and separable if and only if $(\mathbf{B} X$, $)$ is an $\omega$ domain.

\subsection{A computational model for complete fuzzy metric spaces $(X, M, *)$ with $* \geq *_{L}$}

For each $a \in \mathbb{R}$, we shall denote by $a^{+}$the non-negative real number $a \vee 0$.

Given a fuzzy metric space $(X, M, *)$ with $* \geq *_{L}$, define a fuzzy set $\mathcal{M}: \mathbf{B} X \times \mathbf{B} X \times \mathbb{R}^{+} \rightarrow[0,1]$ by

$$
\mathcal{M}((x, r),(y, s), 0)=0
$$

for all $(x, r),(y, s) \in \mathbf{B} X$, and

$\mathcal{M}((x, r),(y, s), t)=\left[\left(M\left(x, y,(t+r-s)^{+}\right) \wedge(1-(|r-s| \wedge 1))\right)+((r-s) \wedge 1)\right]^{+}$

for all $(x, r),(y, s) \in \mathbf{B} X$ and $t>0$.

Remark 4.10. Note that if $t+r-s \leq 0$, then $M\left(x, y,(t+r-s)^{+}\right)=0$ and $r<s$, so $\mathcal{M}((x, r),(y, s), t)=0$.

We omit the easy proof of the following auxiliary fact.

Lemma 4.11. Let $a, b, c, d \in \mathbb{R}^{+}$. Then 


$$
(a+b-1)^{+} \wedge(c+d-1)^{+} \geq(a \wedge c)+(b \wedge d)-1 .
$$

Now we prove the main result in this section.

Theorem 4.12. Let $(X, M, *)$ be a fuzzy metric space such that $* \geq$ $*_{L}$. Then

(1) $\left(\mathbf{B} X, \mathcal{M}, *_{L}\right)$ is a fuzzy quasi-metric space.

$(2) \sqsubseteq=\leq_{\mathcal{M}}$ on $\mathbf{B} X$.

(3) $(X, M, *)$ and $\left(\operatorname{Max}((\mathbf{B} X, \sqsubseteq)), \mathcal{M}, *_{L}\right)$ are isometric.

(4) The topology induced by $\left(\mathcal{M}, *_{L}\right)$ coincides with the Scott topology of $(\mathbf{B} X, \sqsubseteq)$.

(5) $(X, M, *)$ is complete if and only if $\left(\mathbf{B} X, \mathcal{M}, *_{L}\right)$ is bicomplete.

Proof. (1) We first note that for each $(x, r) \in \mathbf{B} X$ and each $t>0$, we have

$$
\mathcal{M}((x, r),(x, r), t)=M(x, x, t)=1
$$

Now let $(x, r),(y, s) \in \mathbf{B} X$ be such that

$$
\mathcal{M}((x, r),(y, s), t)=\mathcal{M}((y, s),(x, r), t)=1,
$$

for all $t>0$. We show that $(x, r)=(y, s)$. Suppose $r>s$. Then

$\mathcal{M}((y, s),(x, r), t)=1 \Rightarrow\left(M\left(y, x,(t+s-r)^{+}\right) \wedge(1-((r-s) \wedge 1))\right)+s-r=1$ for all $t>0$, so, in particular

$$
1-((r-s) \wedge 1) \geq 1+r-s,
$$

and hence $s-r \geq(r-s) \wedge 1>0$, a contradiction. Therefore $r \leq s$. 
Similarly we show that $s \leq r$, and thus $r=s$. So

$$
\mathcal{M}(x, y, t)=M(x, y, t)
$$

for all $t>0$, which implies that $M(x, y, t)=1$ for all $t>0$. Hence $x=y$. We conclude that $(x, r)=(y, s)$.

Next we show that for $(x, r),(y, s),(z, u) \in \mathbf{B} X$ and $t_{1}, t_{2}>0$, one has

$$
\mathcal{M}\left((x, r),(z, u), t_{1}+t_{2}\right) \geq \mathcal{M}\left((x, r),(y, s), t_{1}\right) *_{L} \mathcal{M}\left((y, s),(z, u), t_{2}\right)
$$

We shall assume that $\mathcal{M}\left((x, r),(y, s), t_{1}\right) *_{L} \mathcal{M}\left((y, s),(z, u), t_{2}\right)>0$, because otherwise the inequality (4.2) is obvious.

Then $\mathcal{M}\left((x, r),(y, s), t_{1}\right)>0$ and $\mathcal{M}\left((y, s),(z, u), t_{2}\right)>0$. So, by Remark $4.10, t_{1}+r-s>0$ and $t_{2}+s-u>0$, and hence $t_{1}+t_{2}+r-u>0$. Therefore

$$
\begin{aligned}
& \mathcal{M}\left((x, r),(y, s), t_{1}\right) *_{L} \mathcal{M}\left((y, s),(z, u), t_{2}\right) \\
= & \left(M\left(x, y, t_{1}+r-s\right) \wedge(1-(|r-s| \wedge 1))\right)+((r-s) \wedge 1) \\
& +\left(M\left(y, z, t_{2}+s-u\right) \wedge(1-(|s-u| \wedge 1))\right)+((s-u) \wedge 1)-1
\end{aligned}
$$

Since

$$
\begin{aligned}
M\left(x, z, t_{1}+t_{2}+r-u\right) & \geq M\left(x, y, t_{1}+r-s\right) *_{L} M\left(y, z, t_{2}+s-u\right) \\
& =\left[M\left(x, y, t_{1}+r-s\right)+M\left(y, z, t_{2}+s-u\right)-1\right]^{+}
\end{aligned}
$$

and

$$
1-(|r-u| \wedge 1) \geq\left[\left(1-(|r-s| \wedge 1)+(1-(|s-u| \wedge 1)-1]^{+}\right.\right.
$$


we deduce that

$$
\begin{aligned}
& \left.M\left(x, z, t_{1}+t_{2}+r-u\right) \wedge(1-(\mid r-u) \mid \wedge 1)\right) \\
\geq \quad & {\left[M\left(x, y, t_{1}+r-s\right)+M\left(y, z, t_{2}+s-u\right)-1\right]^{+} } \\
& \wedge[(1-(|r-s| \wedge 1))+(1-(|s-u| \wedge 1))-1]^{+},
\end{aligned}
$$

so, by Lemma 4.11

$$
\begin{aligned}
& \left.\left(M\left(x, z, t_{1}+t_{2}+r-u\right) \wedge(1-(\mid r-u) \mid \wedge 1)\right)\right) \\
\geq \quad & \left(\left(M\left(x, y, t_{1}+r-s\right) \wedge(1-(|r-s| \wedge 1))\right)\right. \\
& +\left(\left(M\left(y, z, t_{2}+s-u\right) \wedge(1-(|s-u| \wedge 1))\right)-1 .\right.
\end{aligned}
$$

Therefore

$$
\begin{aligned}
& \left.\left(M\left(x, z, t_{1}+t_{2}+r-u\right) \wedge(1-(\mid r-u) \mid \wedge 1)\right)\right)+((r-u) \wedge 1) \\
\geq & \left(\left(M\left(x, y, t_{1}+r-s\right) \wedge(1-(|r-s| \wedge 1))\right)+((r-s) \wedge 1)\right. \\
& +\left(\left(M\left(y, z, t_{2}+s-u\right) \wedge(1-(|s-u| \wedge 1))\right)+((s-u) \wedge 1)-1\right.
\end{aligned}
$$

i.e.,

$$
\mathcal{M}\left((x, r),(z, u), t_{1}+t_{2}\right) \geq \mathcal{M}\left((x, r),(y, s), t_{1}\right) *_{L} \mathcal{M}\left((y, s),(z, u), t_{2}\right)
$$


Finally, fix $(x, r),(y, s) \in \mathbf{B} X$. Since the function $M\left(x, y,_{-}\right): \mathbb{R}^{+} \rightarrow[0,1]$ is left continuous, it immediately follows that the function $\mathcal{M}\left((x, r),(y, s),{ }_{-}\right)$: $\mathbb{R}^{+} \rightarrow[0,1]$ is left continuous.

We conclude that $\left(\mathcal{M}, *_{L}\right)$ is a fuzzy quasi-metric on $\mathbf{B} X$.

(2) Let $(x, r),(y, s) \in \mathbf{B} X$. Then

$$
\begin{aligned}
(x, r) & \left.\sqsubseteq(y, s) \Longleftrightarrow \inf _{t>r-s} M(x, y, t) \geq 1-((r-s) \wedge 1)\right) \\
& \left.\Longleftrightarrow \inf _{t>0} M(x, y, t+r-s) \geq 1-((r-s) \wedge 1)\right) \\
& \Longleftrightarrow \inf _{t>0} \mathcal{M}((x, r),(y, s), t)=1 \Longleftrightarrow(x, r) \leq_{\mathcal{M}}(y, s) .
\end{aligned}
$$

Therefore $\sqsubseteq_{M}=\leq_{\mathcal{M}}$ on $\mathbf{B} X$.

(3) Define $i: X \rightarrow \mathbf{B} X$ by $i(x)=(x, 0)$ for all $x \in X$. Then, for each $x, y \in X$ and $t>0$, we obtain

$$
\mathcal{M}(i(x), i(y), t)=\mathcal{M}((x, 0),(y, 0), t)=M(x, y, t) .
$$

Since $(\operatorname{Max}((\mathbf{B} X, \sqsubseteq))=\{(x, 0): x \in X\}$, we conclude that $i$ is an isometry between $(X, M, *)$ and $\left(\operatorname{Max}((\mathbf{B} X, \sqsubseteq)), \mathcal{M}, *_{L}\right)$.

(4) We first recall ([20, Proposition 7]) that for a metric space $(X, d)$ and $(x, r),(y, s) \in \mathbf{B} X$ it follows that

$$
(x, r) \ll(y, s) \Longleftrightarrow d(x, y)<r-s .
$$

Now we shall prove that $\tau_{\mathcal{M}} \subseteq \sigma(\mathbf{B} X)$, where $\sigma(\mathbf{B} X)$ denotes the Scott topology of $(\mathbf{B} X, \sqsubseteq)$.

Let $(x, r) \in \mathbf{B} X$ and $\varepsilon \in(0,1)$. We wish to show that

$$
\Uparrow\left(x, r+\frac{\varepsilon}{2}\right) \subseteq B_{\mathcal{M}}((x, r), \varepsilon) .
$$


Indeed, take $(y, s) \in \Uparrow(x, r+\varepsilon / 2)$. By Proposition 4.7 and equivalence (4.3) it follows that

$$
d(M)(x, y)<r+\frac{\varepsilon}{2}-s .
$$

If $r+\varepsilon / 2-s<1$, we deduce from (4.4) that

$$
M\left(x, y, r+\frac{\varepsilon}{2}-s\right)>1-\left(r+\frac{\varepsilon}{2}-s\right) .
$$

Hence

$$
\begin{aligned}
M\left(x, y,(\varepsilon+r-s)^{+}\right)+((r-s) \wedge 1) & =M(x, y, \varepsilon+r-s)+r-s \\
& >1-\left(r+\frac{\varepsilon}{2}-s\right)+r-s \\
& =1-\frac{\varepsilon}{2} .
\end{aligned}
$$

Moreover, since, from our hypothesis, $r-s<1$ and, by (4.4), $s-r<$ $\varepsilon / 2$, we deduce that

$$
1-(|r-s| \wedge 1)+((r-s) \wedge 1)=1-|r-s|+r-s>1-\frac{\varepsilon}{2} .
$$

We conclude that

$$
\mathcal{M}((x, r),(y, s)), \varepsilon)>1-\frac{\varepsilon}{2}
$$

i.e., $(y, s) \in B_{\mathcal{M}}((x, r), \varepsilon / 2) \subseteq B_{\mathcal{M}}((x, r), \varepsilon)$.

If $r+\varepsilon / 2-s \geq 1$, we have

$$
(r-s) \wedge 1 \geq\left(1-\frac{\varepsilon}{2}\right) \wedge 1>1-\varepsilon,
$$

SO

$$
\mathcal{M}((x, r),(y, s)), \varepsilon)>1-\varepsilon
$$


i.e., $(y, s) \in B_{\mathcal{M}}((x, r), \varepsilon)$.

We conclude that $\tau_{\mathcal{M}} \subseteq \sigma(\mathbf{B} X)$.

It remains to prove that $\sigma(\mathbf{B} X) \subseteq \tau_{\mathcal{M}}$.

To this end, let $(x, r) \in \mathbf{B} X$ and suppose $(x, r) \in \Uparrow(y, s)$ in $(\mathbf{B} X, \sqsubseteq)$ for some $(y, s) \in \mathbf{B} X$. By Proposition 4.7 and (4.3), there exists $\varepsilon \in(0,1)$ such that

$$
d(M)(y, x)<s-r-2 \varepsilon .
$$

We deduce that $(x, r) \in \Uparrow(x, r+2 \varepsilon) \subseteq \Uparrow(y, s)$ in $\left(\mathbf{B} X, \sqsubseteq_{d(M)}\right)$ and hence in $(\mathbf{B} X, \sqsubseteq)$.

We shall show that $B_{\mathcal{M}}((x, r), \varepsilon, \varepsilon) \subseteq \Uparrow(x, r+2 \varepsilon)$.

Indeed, choose $(z, u) \in B_{\mathcal{M}}((x, r), \varepsilon, \varepsilon)$.

If $r-u \geq 1-\varepsilon$, we deduce that

$$
d(M)(x, z) \leq 1 \leq r+\varepsilon-u<r+2 \varepsilon-u
$$

By $(4.3),(z, u) \in \Uparrow(x, r+2 \varepsilon)$ in $\left(\mathbf{B} X, \sqsubseteq_{d(M)}\right)$, and thus $(z, u) \in \Uparrow(x, r+2 \varepsilon)$ in $(\mathbf{B} X, \sqsubseteq)$ by Proposition 4.7 .

Now suppose $r-u<1-\varepsilon$. Then we have $\varepsilon+r-u>0$, since otherwise one has

$$
M\left(x, z,(\varepsilon+r-u)^{+}\right)=0,
$$

and hence

$$
\mathcal{M}((x, r),(z, u), \varepsilon)=((r-u) \wedge 1)^{+}=0,
$$

which contradicts our assumption that $(z, u) \in B_{\mathcal{M}}((x, r), \varepsilon, \varepsilon)$.

Thus, from $r-u<1-\varepsilon, \varepsilon+r-u>0$ and the fact that $\mathcal{M}((x, r),(z, u), \varepsilon)>$ $1-\varepsilon$, we deduce that

$$
M(x, z, r+\varepsilon-u)>1-\varepsilon-((r-u) \wedge 1)=1-(r+\varepsilon-u) .
$$

Therefore

$$
d(M)(x, z)<r+\varepsilon-u<r+2 \varepsilon-u \text {. }
$$


We conclude that $(z, u) \in \Uparrow(x, r+2 \varepsilon)$ in $\left(\mathbf{B} X, \sqsubseteq_{d(M)}\right)$ and thus in $(\mathbf{B} X, \sqsubseteq$ ) .

Consequently $\sigma(\mathbf{B} X) \subseteq \tau_{\mathcal{M}}$.

(5) Suppose that $(X, M, *)$ is complete and let $\left(\left(x_{n}, r_{n}\right)\right)_{n}$ be a Cauchy sequence in the fuzzy metric space $\left(\mathbf{B} X, \mathcal{M}^{i}, *_{L}\right)$.

Given $\varepsilon \in(0,1)$ there is $n_{0}$ such that

$$
\mathcal{M}\left(\left(x_{n}, r_{n}\right),\left(x_{m}, r_{m}\right), \varepsilon\right)>1-\varepsilon
$$

whenever $n, m \geq n_{0}$.

If there exist $n, m \geq n_{0}$ satisfying $r_{m}-r_{n} \geq 1$, we deduce that

$$
\mathcal{M}\left(\left(x_{n}, r_{n}\right),\left(x_{m}, r_{m}\right), \varepsilon\right)=0
$$

so that $\left|r_{n}-r_{m}\right|<1$ eventually.

Hence, and assuming without loss of generality that $r_{m} \geq r_{n}$, we obtain by (4.5) that

$$
\begin{aligned}
1-\left(\left|r_{n}-r_{m}\right| \wedge 1\right)+\left(\left(r_{n}-r_{m}\right) \wedge 1\right) & =1-\left(r_{m}-r_{n}\right)+r_{n}-r_{m} \\
& >1-\varepsilon,
\end{aligned}
$$

eventually. Therefore $2\left|r_{n}-r_{m}\right|<\varepsilon$ eventually, so $\left(r_{n}\right)_{n}$ is a Cauchy sequence in $\mathbb{R}^{+}$with respect to the usual metric. There exists $r \in \mathbb{R}^{+}$such that $\lim _{n} r_{n}=r$.

Next we show that $\left(x_{n}\right)_{n}$ is a Cauchy sequence in $(X, M, *)$. In fact, from (4.5) and assuming, without loss of generality, that $r_{m}<r_{n}<\varepsilon+r_{m}$, we deduce that

$$
M\left(x_{n}, x_{m}, \varepsilon+r_{n}-r_{m}\right)+\left(r_{n}-r_{m}\right)>1-\varepsilon,
$$

eventually. Since $M\left(x_{n}, x_{m}, 2 \varepsilon\right) \geq M\left(x_{n}, x_{m}, \varepsilon+r_{n}-r_{m}\right)$, it follows that

$$
M\left(x_{n}, x_{m}, 2 \varepsilon\right)>1-\varepsilon-\left(r_{n}-r_{m}\right)>1-2 \varepsilon,
$$


eventually.

We have shown that $\left(x_{n}\right)_{n}$ is a Cauchy sequence in $(X, M, *)$. Since it is complete, there exists $x \in X$ such that $\left(x_{n}\right)_{n}$ converges to $x$ with respect to $\tau_{M}$.

Now it is routine to check that $\left(\left(x_{n}, r_{n}\right)\right)_{n}$ converges to $(x, r)$ in the fuzzy metric space $\left(\mathbf{B} X, \mathcal{M}^{i}, *_{L}\right)$. We conclude that $\left(\mathbf{B} X, \mathcal{M}, *_{L}\right)$ is bicomplete.

The converse is almost obvious. Indeed, let $\left(x_{n}\right)_{n}$ be a Cauchy sequence in $(X, M, *)$. Then $\left(\left(x_{n}, 0\right)\right)_{n}$ is a Cauchy sequence in $\left(\mathbf{B} X, \mathcal{M}^{i}, *_{L}\right)$. Let $(x, 0) \in \mathbf{B} X$ such that $\left(\left(x_{n}, 0\right)\right)_{n}$ converges to $(x, 0)$ with respect to $\tau_{\mathcal{M}^{i}}$. Then $\left(x_{n}\right)_{n}$ converges to $x$ with respect to $\tau_{M}$, so $(X, M, *)$ is complete.

Corollary 4.13. Every complete fuzzy metric space $(X, M, *)$, with $* \geq$ $*_{L}$, has a computational model. 


\section{Chapter 5}

\section{Fixed point theorems for $\varphi$-contractions on fuzzy metric spaces with application to the intuitionistic setting}

\section{$5.1 \quad$ Introduction}

In the last years several authors have studied the existence of fixed points for some kinds of $\varphi$-contractive mappings in fuzzy metric spaces, or, equivalently, in Menger spaces (see. e.g. [1, 15, 27, 40, 49, 70]). Recently, Huang, Zhu and Wen [38] have obtained, among other results, an extension of Golet's fixed point theorem [27, Theorem 3] to intuitionistic fuzzy metric spaces.

Here we shall obtain two fixed point theorems for a kind of $\varphi$-contractive mappings on complete fuzzy metric spaces from which we easily deduce fixed point results for complete intuitionistic fuzzy metric spaces. Such results improve in several directions the main fixed point theorem of [38], and also provide a simplified proof of it.

Finally, we present a version for ordered complete fuzzy metric spaces of 
the main fixed point theorem obtained in Section 5.2.

The results given in Sections 5.2 and 5.3 are contained in the paper by L.A. Ricarte and S. Romaguera, "On $\varphi$-contractions in fuzzy metric spaces with application to the intuitionistic setting", accepted in Iranian Journal of Fuzzy Systems (cf. [58]), while the ones given in Section 5.4 are contained in the paper by L.A. Ricarte, S. Romaguera and P. Tirado, "A fixed point theorem for generalized contractions on ordered complete fuzzy metric spaces", published in the Proceedings of the Conference on Applied Topology WiAT'13 (cf. [59]).

\subsection{Fixed point theorems for complete fuzzy metric spaces}

In [46] Matkowski proved his celebrated (partial) generalization of the BoydWong fixed point theorem [12].

Theorem 5.1 (Matkowski [46]). If $f$ is a self mapping on a complete metric space $(X, d)$ and there exists a non-decreasing function $\varphi: \mathbb{R}^{+} \rightarrow \mathbb{R}^{+}$ satisfying $\lim _{n \rightarrow \infty} \varphi^{n}(t)=0$ for all $t>0$, and $d(f x, f y) \leq \varphi(d(x, y))$ for all $x, y \in X$, then $f$ has a fixed point.

Recently, Jachymski [40] obtained a probabilistic version of Matkowski's theorem by using continuous t-norms of Hadžić type (or $h$-type) [35] and an appropriate notion of $\varphi$-contraction.

In our main result we shall prove a fuzzy metric version of Matkowski's theorem with a different approach to the ones given in [40]. In fact, our notion of $\varphi$-contraction is motivated by the recent work of Huang, Zhu and Wen [38], where the authors extended the notion of C-contraction, as given 
by Hicks [37], to intuitionistic fuzzy metric spaces and obtained some fixed point theorems in this framework.

Definition 5.2. A fuzzy $\varphi$-contraction on a fuzzy metric space $(X, M, *)$ is a mapping $f: X \rightarrow X$ such that there is a non-decreasing function $\varphi$ : $\mathbb{R}^{+} \rightarrow \mathbb{R}^{+}$satisfying $\lim _{n \rightarrow \infty} \varphi^{n}(t)=0$ for all $t>0$, and

$$
M(x, y, t)>1-t \Rightarrow M(f x, f y, \varphi(t))>1-\varphi(t)
$$

for all $x, y \in X$ and $t>0$.

Recall that if $\varphi: \mathbb{R}^{+} \rightarrow \mathbb{R}^{+}$is non-decreasing and satisfies $\lim _{n \rightarrow \infty} \varphi^{n}(t)=$ 0 for all $t>0$, then $\varphi(t)<t$ for all $t>0$.

Theorem 5.3. Every fuzzy $\varphi$-contraction on a complete fuzzy metric space has a unique fixed point.

Proof. Let $(X, M, *)$ be a complete fuzzy metric space and let $f: X \rightarrow X$ be a $\varphi$-contraction on $(X, M, *)$.

Then, there is a function $\varphi$ verifying the conditions of Definition 5.2.

Take $t_{0}>1$. Then, for each $x, y \in X$ we have $M\left(x, y, t_{0}\right)>1-t_{0}$, so

$$
M\left(f x, f y, \varphi\left(t_{0}\right)\right)>1-\varphi\left(t_{0}\right) .
$$

Repeating the process, we obtain that

$$
M\left(f^{n} x, f^{n} y, \varphi^{n}\left(t_{0}\right)\right)>1-\varphi^{n}\left(t_{0}\right)
$$

for all $x, y \in X$ and $n \in \mathbb{N}$.

Now choose $x_{0} \in X$. We show that $\left(f^{n} x_{0}\right)_{n}$ is a Cauchy sequence in $(X, M, *)$. 
Indeed, given $\varepsilon \in(0,1)$ and $t>0$, there exists $n_{\varepsilon} \in \mathbb{N}$ such that $\varphi^{n}\left(t_{0}\right)<$ $\min \{\varepsilon, t\}$ for all $n \geq n_{\varepsilon}$.

Let $m>n \geq n_{0}$. Then $m=n+k$ for some $k \in \mathbb{N}$, and thus

$$
\begin{aligned}
M\left(f^{n} x_{0}, f^{m} x_{0}, t\right) & =M\left(f^{n} x_{0}, f^{n} f^{k} x_{0}, t\right) \\
& \geq M\left(f^{n} x_{0}, f^{n} f^{k} x_{0}, \varphi^{n}\left(t_{0}\right)\right) \\
& >1-\varphi^{n}\left(t_{0}\right) \\
& >1-\varepsilon .
\end{aligned}
$$

Consequently $\left(f^{n} x_{0}\right)_{n}$ is a Cauchy sequence in $(X, M, *)$.

So there is $z \in X$ such that the sequence $\left(f^{n} x_{0}\right)_{n}$ converges to $z$ for $\tau_{M}$, i.e., $\lim _{n \rightarrow \infty} M\left(z, f^{n} x_{0}, t\right)=1$ for all $t>0$.

Next we show that $z$ is the unique fixed point of $f$.

Indeed, given $t>0$ and $\varepsilon \in(0, t)$ there exists $n_{\varepsilon} \in \mathbb{N}$ such that $M\left(z, f^{n} x_{0}, \varepsilon\right)>$ $1-\varepsilon$ for all $n \geq n_{\varepsilon}$. Hence

$$
M\left(f z, f^{n+1} x_{0}, \varphi(\varepsilon)\right)>1-\varphi(\varepsilon)
$$

for all $n \geq n_{\varepsilon}$. Since $\varphi(\varepsilon)<\varepsilon<t$, we deduce that

$$
\begin{aligned}
M\left(f z, f^{n+1} x_{0}, t\right) & \geq M\left(f z, f^{n+1} x_{0}, \varepsilon\right) \geq M\left(f z, f^{n+1} x_{0}, \varphi(\varepsilon)\right) \\
& >1-\varphi(\varepsilon)>1-\varepsilon
\end{aligned}
$$

for all $n \geq n_{\varepsilon}$.

Hence $\lim _{n \rightarrow \infty} M\left(f z, f^{n} x_{0}, t\right)=1$ for all $t>0$, and thus, $z=f z$.

Finally, suppose that $u \in X$ satisfies $u=f u$. Then

$$
M\left(u, z, \varphi^{n}\left(t_{0}\right)\right)=M\left(f^{n} u, f^{n} z, \varphi^{n}\left(t_{0}\right)\right) \geq 1-\varphi^{n}\left(t_{0}\right)
$$


for all $n \in \mathbb{N}$.

Given an arbitrary $t>0$, there is $n_{\varepsilon} \in \mathbb{N}$ such that $\varphi^{n}\left(t_{0}\right)<t$ for all $n \geq n_{\varepsilon}$. Hence

$$
M(u, z, t) \geq 1-\varphi^{n}\left(t_{0}\right)
$$

for all $n \geq n_{\varepsilon}$.

Since $\lim _{n \rightarrow \infty} \varphi^{n}\left(t_{0}\right)=0$, we conclude that $M(u, z, t)=1$. Since $t$ is arbitrary, it follows that $u=z$. The proof is complete.

Motivated by the notions of a fuzzy contractive sequence and of a fuzzy contractive mapping given in [33], Radu introduced in [54] the notion of a strict B-contraction for which he obtained a nice fixed point theorem for any complete fuzzy metric space $(X, M, *)$ satisfying $* \geq *_{L}$ (recall that by $*_{L}$ we denote the Lukasiewicz t-norm).

Later on, Mihet [47] generalized the notion of a strict B-contraction, introducing the concept of $(\varphi-k)$-B contraction.

Then, he proved [47, Theorem 3.11] that if $(X, M *)$ is a complete fuzzy metric space and $f: X \rightarrow X$ is a $(\varphi-k)$-B contraction satisfying $M(x, f x, t)>$ 0 for some $x \in X$ and $t>0$, then $f$ has a (non necessarily unique) fixed point in $X$.

Let us recall [47, Definition 3.6] that a self-mapping $f$ on a fuzzy metric space $(X, M, *)$ is said to be a $(\varphi-k)$-B contraction if there is a function $\varphi \in \Phi$ and a constant $k \in(0,1)$ satisfying the following condition

$$
M(x, y, t)>1-\lambda \Rightarrow M(f x, f y, k t)>1-\varphi(\lambda),
$$

for all $x, y \in X, t>0$ and $\lambda \in(0,1)$, where by $\Phi$ is denoted the class of all functions $\varphi:(0,1) \rightarrow(0,1)$ such that $\varphi$ is an increasing bijection satisfying $\varphi(\lambda)<\lambda$ for all $\lambda \in(0,1)$. 
Note that if $\varphi \in \Phi$, then $\lim _{n \rightarrow \infty} \varphi^{n}(\lambda)=0$ for all $\lambda \in(0,1)$.

The following two easy examples illustrate the differences between Theorem 5.3 and Mihet's theorem cited above.

Example 5.4. Let $X=\{0,1\}$, and $(M, \wedge)$ be the complete fuzzy metric on $X$ given by $M(x, x, t)=1$ for all $x \in X$ and $t>0$, and $M(x, y, t)=0$ otherwise.

Define $f: X \rightarrow X$ by $f x=x$ for all $x \in X$. Clearly $f$ is a $(\varphi-k)$-B contraction for every $\varphi \in \Phi$ and $k \in(0,1)$. So, the hypotheses of Mihet's theorem are verified.

However $f$ is not a fuzzy $\varphi$-contraction because $f$ has no a unique fixed point. In fact, if $f$ was a fuzzy $\varphi$-contraction for some $\varphi$ satisfying the conditions of Definition 5.2, then, from $M(0,1, t)>1-t$ we would have $M(f 0, f 1, \varphi(t))>1-\varphi(t)$.

Hence $\varphi(t)>1$ for all $t>1$, and thus $\lim _{n \rightarrow \infty} \varphi^{n}(t) \geq 1$ whenever $t>1$, which contradicts the definition of fuzzy $\varphi$-contraction. So, indeed, we cannot apply Theorem 5.3 to this example.

Example 5.5. Let $X=[0,1]$, and $(M, \wedge)$ be the complete fuzzy metric on $X$ given by $M(x, y, t)=|x-y|$ if $t>0$ and $|x-y|<t$, and $M(x, y, t)=0$ otherwise.

Define $f: X \rightarrow X$ by $f x=x / 2$ for all $x \in X$, and $\varphi: \mathbb{R}^{+} \rightarrow \mathbb{R}^{+}$ by $\varphi(0)=0, \varphi(t)=1 /(n+1)$ whenever $t \in(1 /(n+1), 1 / n], n \in \mathbb{N}$, and $\varphi(t)=t / 2$ whenever $t>1$.

We prove that $f$ is a fuzzy $\varphi$-contraction on $(X, M, *)$.

It is clear that $\varphi$ is non-decreasing with $t / 2 \leq \varphi(t)<t$ for all $t>0$. Moreover, it is easy to check that $\lim _{n \rightarrow \infty} \varphi^{n}(t)=0$ for all $t>0$.

Let $M(x, y, t)>1-t$. If $M(x, y, t)=0$, it follows that $t>1$ and $|x-y| \geq$ $t$, which is not possible because $x, y \in[0,1]$. Hence, we shall suppose that 
$M(x, y, t)=1$. Then $|x-y|<t$, so $|x-y|<2 \varphi(t)$, i.e., $M(f x, f y, \varphi(t))=1$. We have shown that $f$ is a fuzzy $\varphi$-contraction on $(X, M, \wedge)$, and thus the hypotheses of Theorem 5.3 are verified.

However, the restriction of $\varphi$ to $(0,1)$ does not belong to the class $\Phi$ because it is not one-to-one on $(0,1)$.

Remark 5.6. Note that both in Example 5.4 and Example 5.5, there exist $x, y \in X$ and $t>0$ for which $M(x, y, t)=0$.

Next we give other two examples that also allow us to distinguish between Theorem 5.3 and Mihet's theorem, and for which the considered fuzzy metric space $(X, M, *)$ verifies that $M(x, y, t)>0$ for all $x, y \in X$ and $t>0$.

Example 5.7. Let $X=\mathbb{R}^{+}$and let $d$ be the Euclidean metric on $X$. Then $\left(X, M_{d}, \wedge\right)$ is a complete fuzzy metric space and $M_{d}(x, y, t)>0$ for all $x, y \in X$ and $t>0$.

Define $f: X \rightarrow X$ by $f x=x / 4$ for all $x \in X$ and $\varphi: \mathbb{R}^{+} \rightarrow \mathbb{R}^{+}$by $\varphi(t)=t /(2-t)$ whenever $t \in[0,1)$ and $\varphi(t)=1$ whenever $t \geq 1$.

Since $\lim _{n \rightarrow \infty} \varphi^{n}(t)=1$ for all $t \geq 1$ we deduce that $f$ is not a fuzzy $\varphi$-contraction, so we cannot apply Theorem 5.3 to this case.

However, it is easy to check that $f$ is a $(\psi-k)$-B contraction for $\psi$ the restriction of $\varphi$ to $(0,1)$ and $k=1 / 2$. Moreover $M_{d}(x, f x, t)>0$ for $x \in X \backslash\{0\}$ and $t>0$, so the hypotheses of Mihet's theorem are verified.

Example 5.8. Let $\left(X, M_{d}, \wedge\right)$ defined as in Example 5.7, let $f x=0$ for all $x \in X$ and $\varphi: \mathbb{R}^{+} \rightarrow \mathbb{R}^{+}$defined as in Example 5.5.

Obviously, $f$ is a $\varphi$-contraction, and hence we can apply Theorem 5.3. However, the restriction of $\varphi$ to $(0,1)$ does not belong to the class $\Phi$ (see Example 5.5). 
Next we generalize our notion of fuzzy $\varphi$-contraction as follows.

Definition 5.9 (compare $[27,38])$. A fuzzy $(g, \varphi)$-contraction on a fuzzy metric space $(X, M, *)$ is a mapping $f: X \rightarrow X$ such that there exist a bijective mapping $g: X \rightarrow X$ and a non-decreasing function $\varphi: \mathbb{R}^{+} \rightarrow \mathbb{R}^{+}$ satisfying $\lim _{n \rightarrow \infty} \varphi^{n}(t)=0$ for all $t>0$, and

$$
M(g x, g y, t)>1-t \Rightarrow M(f x, f y, \varphi(t))>1-\varphi(t)
$$

for all $x, y \in X$ and $t>0$.

Now we extend Theorem 5.3 to the case of fuzzy $(g, \varphi)$-contractions. Actually, this will be done by a direct application of that theorem.

It is interesting to point out that Mihet [48] already used a similar approach to deduce the fixed point theorem proved by Golet in [27, Theorem 3] from the ones proved by Radu in [53] for C-contractions.

Theorem 5.10. Let $(X, M, *)$ be a complete fuzzy metric space. If $f: X \rightarrow X$ is a fuzzy $(g, \varphi)$-contraction on $(X, M, *)$, then there is a unique $z \in X$ such that $f z=g z$.

Proof. Since $f$ is a fuzzy $(g, \varphi)$-contraction, there exist a bijective mapping $g: X \rightarrow X$ and function $\varphi$ verifying the conditions of Definition 5.9.

Define $h:=g^{-1}$. Then $g(h)=1_{X}$. Clearly $f(h)$ is a fuzzy $\varphi$-contraction on $(X, M, *)$.

Indeed, let $M(x, y, t)>1-t$. Since $x=g(h) x$ and $=g(h) y$, it follows that $M(f(h) x, f(h) y, \varphi(t))>1-\varphi(t)$.

Therefore, by Theorem 5.3, there exists a unique $u \in X$ such that $u=$ $f(h) u$. Let $z=h u$. Then $f z=f(h) u=u=g(h) u=g z$. 
Finally, if $f v=g v$, it follows that $f h g v=g v$, so $g v$ is a fixed point of $f h$, and thus $u=g v$. Since $u=g z$ and $g$ is injective we conclude that $v=z$. This completes the proof.

\subsection{Application to intuitionistic fuzzy metric spaces}

The fixed point theorems obtained in Section 2 admit an easy and natural extension to the intuitionistic framework.

We first recall some pertinent concepts and properties.

A continuous t-conorm [72] is a binary operation $\diamond:[0,1] \times[0,1] \rightarrow[0,1]$ which satisfies the following conditions:

(i) $\diamond$ is associative and commutative;

(ii) $a \diamond 0=a$ for every $a \in[0,1]$;

(iii) $a \diamond b \leq c \diamond d$ whenever $a \leq c$ and $b \leq d$, and $a, b, c, d \in[0,1]$;

(iv) $\diamond$ is continuous.

It is well known, and easy to see, that for each continuous t-conorm $\diamond$, one has $\wedge \leq \vee \leq \diamond$, where, as usual $\vee$ denotes the continuous t-conorm of maximum.

It is also well known that if $*$ is a continuous t-norm (respectively, a continuous t-conorm), then $*^{\prime}$ is a continuous t-conorm (respectively, a continuous t-norm), where $a *^{\prime} b=1-[(1-a) *(1-b)]$ for all $a, b \in[0,1]$.

Definition $5.11([3])$. An intuitionistic fuzzy metric on a (non-empty) set $X$ is a 4 -tuple $(M, N, *, \diamond)$ such that $(M, *)$ is a fuzzy metric on $X, \diamond$ is a continuous t-conorm and $N$ is a fuzzy set in $X \times X \times \mathbb{R}^{+}$such that for all $x, y, z \in X$ : 
(N1) $N(x, y, 0)=1$;

(N2) $N(x, y, t)=0$ for all $t>0$ if and only if $x=y$;

(N3) $N(x, y, t)=N(y, x, t)$ for all $t>0$;

(N4) $N(x, y, t) \diamond N(y, z, s) \geq N(x, z, t+s)$ for all $t, s \geq 0$;

(N5) $N\left(x, y,{ }_{-}\right): \mathbb{R}^{+} \rightarrow[0,1]$ is left continuous;

$(\mathrm{MN}) M(x, y, t)+N(x, y, t) \leq 1$.

An intuitionistic fuzzy metric space is a 5 -tuple $(X, M, N, *, \diamond)$ such that $X$ is a (non-empty) set and $(M, N, *, \diamond)$ is an intuitionistic fuzzy metric on $X$.

We point out that Park gave in [52] (see also [30, 69]) a more strict notion of intuitionistic fuzzy metric space which is based on the notion of a fuzzy metric due to George and Veeramani [23]. Nevertheless, Park's results and concepts which will be consider here are also valid for intuitionistic fuzzy metric spaces as defined above.

Park showed in [52] (see also [64, 65]) that, as for fuzzy metric spaces, every intuitionistic fuzzy metric $(M, N, *, \diamond)$ on a (non-empty) set $X$ induces a topology $\tau_{(M . N)}$ on $X$ which has as a base the family of open balls $\left\{B_{(M, N)}(x, \varepsilon, t): \varepsilon \in(0,1), t>0\right\}$, where

$$
B_{(M, N)}(x, \varepsilon, t)=\{y \in X: M(x, y, t)>1-\varepsilon \text { and } N(x, y, t)<\varepsilon\}
$$

for all $x \in X, t>0$ and $\varepsilon \in(0,1)$.

Similarly to [52], we say that a sequence $\left(x_{n}\right)_{n}$ in an intuitionistic fuzzy metric space $(X, M, N, *, \diamond)$ is a Cauchy sequence if for each $t>0$ and each $\varepsilon \in(0,1)$ there exists $n_{0} \in \mathbb{N}$ such that $M\left(x_{n}, x_{m}, t\right)>1-\varepsilon$ and $N\left(x_{n}, x_{m}, t\right)<\varepsilon$ for all $n, m \geq n_{0} .(X, M, N, *, \diamond)$ is called complete if every Cauchy sequence converges with respect to $\tau_{(M, N)}$. 
As direct consequences of condition (MN) in Definition 5.11 we have the following easy but crucial facts.

Proposition 5.12 (see e.g. $[30,64,65]$ ). Let $(X, M, N, *, \diamond)$ be an intuitionistic fuzzy metric space. Then $B_{(M, N)}(x, \varepsilon, t)=B_{M}(x, \varepsilon, t)$ for all $x \in X, t>0$ and $\varepsilon \in(0,1)$. Hence the topologies $\tau_{(M . N)}$ and $\tau_{M}$ coincide on $X$.

Proposition 5.13 (see e.g. [65]). Let $(X, M, N, *, \diamond)$ be an intuitionistic fuzzy metric space. Then:

(a) A sequence in $X$ is a Cauchy sequence in $(X, M, N, *, \diamond)$ if and only if it is a Cauchy sequence in $(X, M, *)$.

(b) $(X, M, N, *, \diamond)$ is complete if and only if $(X, M, *)$ is complete.

The following is a typical example of an intuitionistic fuzzy metric space.

Example 5.14 ([3, 38, 52]). Let $(X, d)$ be a metric space. Define $M_{d}, N_{d}$ : $X \times X \times \mathbb{R}^{+} \rightarrow[0,1]$ by $M_{d}(x, y, 0)=0, N_{d}(x, y, 0)=1$, and

$$
M_{d}(x, y, t)=\frac{t}{t+d(x, y)}, \quad N_{d}(x, y, t)=\frac{d(x, y)}{t+d(x, y)}
$$

for all $x, y \in X$ and $t>0$.

Then $\left(X, M_{d}, N_{d}, *, \diamond\right)$ is an inuitionistic fuzzy metric space for each continuous t-norm $*$ and each continuous t-conorm $\diamond$, with $\tau_{d}=\tau_{(M, N)}$.

Moreover, from Proposition 5.13 and the well-known fact that $\left(X, M_{d}, *\right)$ is complete if and only $(X, d)$ is complete, it follows that $\left(X, M_{d}, N_{d}, *, \diamond\right)$ is complete if and only if $(X, d)$ is complete.

Now the following results are direct consequences of Proposition 5.13, and Theorems 5.3 and 5.10, respectively. 
Theorem 5.15. Let $(X, M, N, *, \diamond)$ be a complete intuitionistic fuzzy metric space. If $f: X \rightarrow X$ is a fuzzy $\varphi$-contraction on the fuzzy metric space $(X, M, *)$, then $f$ has a unique fixed point.

Theorem 5.16. Let $(X, M, N, *, \diamond)$ be a complete intuitionistic fuzzy metric space. If $f: X \rightarrow X$ is a fuzzy $(g, \varphi)$-contraction on the fuzzy metric space $(X, M, *)$, then there is a unique $z \in X$ such that $f z=g z$.

In [28] Grabiec introduced the following notion of completeness for fuzzy metric spaces, in order to obtain fuzzy extensions of the classical Banach contraction principle and the Edelstein fixed point theorem, respectively.

Definition $5.17([28])$. (a) A sequence $\left(x_{n}\right)_{n}$ in a fuzzy metric space $(X, M, *)$ is called G-Cauchy if for each $p \in \mathbb{N}$ and each $t>0, \lim _{n \rightarrow \infty} M\left(x_{n}, x_{n+p}, t\right)=$ 1.

(b) A fuzzy metric space is called G-complete if every G-Cauchy sequence converges.

Alaca, Turkoglu and Yildiz [3] generalized Definition 5.17 to the intuitionistic setting as follows.

Definition $5.18([3])$. (a) A sequence $\left(x_{n}\right)_{n}$ in an intuitionistic fuzzy metric space $(X, M, N, *, \diamond)$ is called G-Cauchy if for each $p \in \mathbb{N}$ and each $t>0, \lim _{n \rightarrow \infty} M\left(x_{n}, x_{n+p}, t\right)=1$ and $\lim _{n \rightarrow \infty} N\left(x_{n}, x_{n+p}, t\right)=0$.

(b) An intuitionistic fuzzy metric space $(X, M, N, *, \diamond)$ is called G-complete if for every G-Cauchy sequence $\left(x_{n}\right)_{n}$ there is $x \in X$ such that $\lim _{n \rightarrow \infty} M\left(x, x_{n}, t\right)=$ 1 and $\lim _{n \rightarrow \infty} N\left(x, x_{n}, t\right)=0$ for all $t>0$.

Similarly to Proposition 5.13 we have the following. 
Proposition 5.19 ([64, Propositions 1 and 2$])$. Let $(X, M, N, *, \diamond)$ be an intuitionistic fuzzy metric space. Then:

(a) A sequence in $X$ is a $G$-Cauchy sequence in $(X, M, N, *, \diamond)$ if and only if it is a G-Cauchy sequence in $(X, M, *)$.

(b) $(X, M, N, *, \diamond)$ is G-complete if and only if $(X, M, *)$ is G-complete.

Obviously, every Cauchy sequence in a fuzzy metric space is a G-Cauchy sequence, and hence, every G-complete fuzzy metric space is complete. So, by Propositions 5.13 and 5.19, every G-complete intuitionistic fuzzy metric space is complete.

The converse is not true, in general. In fact, there exist compact, and hence complete, fuzzy metric spaces that are not G-complete [76]. Furthermore, if we denote by $e$ the Euclidean metric on $\mathbb{R}$, then $\left(\mathbb{R}, M_{e}, *\right)$ provides a distinguished example of a complete fuzzy metric space that is not G-complete (see [77]). Hence, by Proposition 5.19, $\left(\mathbb{R}, M_{e}, N_{e}, *, \diamond\right)$ is a complete intuitionistic fuzzy metric space that is not G-complete.

Huang, Zhu and Wen discussed in [38] the existence of fixed points for $(g, \varphi)$-contractions in the realm of G-complete intuitionistic fuzzy metric spaces. To this end, they introduced the following notion.

Definition 5.20 ([38, Definition 11]). An intuitionistic fuzzy $(g, \varphi)$ contraction on an intuitionistic fuzzy metric space $(X, M, N, *, \diamond)$ is a mapping $f: X \rightarrow X$ such that there exist a bijective mapping $g: X \rightarrow X$ and a non-decreasing right continuous function $\varphi: \mathbb{R}^{+} \rightarrow \mathbb{R}^{+}$satisfying $\lim _{n \rightarrow \infty} \varphi^{n}(t)=0$ for all $t>0$, and

$$
M(g x, g y, t)>1-t \Rightarrow M(f x, f y, \varphi(t))>1-\varphi(t)
$$

and

$$
N(g x, g y, t)<t \Rightarrow N(f x, f y, \varphi(t))<\varphi(t)
$$


for all $x, y \in X$ and $t>0$.

Then, they proved, among other, the following main result.

Theorem 5.21 ([38, Theorem 1]). Let $(X, M, N, *, \diamond)$ be a G-complete intuitionistic fuzzy metric space. If $f: X \rightarrow X$ is an intuitionistic fuzzy $(g, \varphi)$-contraction on $(X, M, N, *, \diamond)$, then there is a unique $z \in X$ such that $f z=g z$.

Note that Theorem 5.21 is a direct consequence of Theorem 5.16. In fact, G-completeness can be relaxed to completeness, and both right continuity of $\varphi$ and the contractive condition of $N$ in Definition 5.20 can be omitted.

\subsection{A version for ordered fuzzy metric spaces}

Motivated by the problem of the resolution of certain linear and nonlinear matrix equations, Ran and Reurings proved, in [55], a version of the classical Banach fixed point theorem to ordered complete metric spaces. Independently, Nieto and Rodríguez-López [50] obtained a slightly different version to the one given by Ran and Reurings and presented applications of their result to the existence of a unique solution for a first-order ordinary differential equation with periodic boundary conditions. In Theorem 5.22 below we present these results in a unified way.

Let us recall that an ordered metric space is a triple $(X, \preceq, d)$ such that $\preceq$ is a (partial) order on $X$ and $(X, d)$ is a metric space. If, in addition, $(X, d)$ is complete we say that $(X, \preceq, d)$ is an ordered complete metric space.

An ordered metric space $(X, \preceq, d)$ is called regular if for any nondecreasing sequence $\left(x_{n}\right)_{n \in \omega}$ for $\preceq$, which converges to some $z \in X$ it follows $x_{n} \preceq z$ for all $n \in \omega$. 
Theorem $5.22([50,55])$. Let $(X, \preceq, d)$ be an ordered complete metric space and $f: X \rightarrow X$ a nondecreasing mapping for $\preceq$, such that there exists $a \in(0,1)$ with

$$
d(f x, f y) \leq a d(x, y)
$$

for all $x, y \in X$ satisfying $x \preceq y$.

If there is $x_{0} \in X$ such that $x_{0} \preceq f x_{0}$, and $f$ is continuous or $(X, \preceq, d)$ is regular, then $f$ has a fixed point.

Later on, Agarwal, El-Gebeily and O'Regan [9] proved an ordered version of the well-known fixed point theorem of Matkowski [46], generalizing in this way Theorem 5.22 above (see e.g. [8, 11, 16, 39, 51] for further contributions with applications, in this framework).

Theorem $5.23([9])$. Let $(X, \preceq, d)$ be an ordered complete metric space and $f: X \rightarrow X$ a nondecreasing map for $\preceq$, such that

$$
d(f x, f y) \leq \varphi(d(x, y))
$$

for all $x, y \in X$ with $x \preceq y$, where $\varphi: \mathbb{R}^{+} \rightarrow \mathbb{R}^{+}$is a nondecreasing function satisfying $\lim _{n \rightarrow \infty} \varphi^{n}(t)=0$ for all $t>0$.

If there is $x_{0} \in X$ such that $x_{0} \preceq f x_{0}$, and $f$ is continuous or $(X, \preceq, d)$ is regular, then $f$ has a fixed point. Moreover, the set of fixed points of $f$ is a singleton if and only it is well-ordered.

The problem of obtaining fuzzy metric counterparts of fixed point theorems on ordered complete metric spaces has been discussed by some authors (see e.g. $[4,6,7,17]$ ).

By an ordered fuzzy metric space we mean a 4 -tuple $(X, \preceq, M, *)$ such that $\preceq$ is a partial order on $X$ and $(X, M, *)$ is a fuzzy metric space. If, in addition, $(X, M, *)$ is a complete fuzzy metric space, we say that $(X, \preceq, M, *)$ is an ordered complete fuzzy metric space 
An ordered fuzzy metric space $(X, \preceq, M, *)$ is called regular if for any nondecreasing sequence $\left(x_{n}\right)_{n \in \omega}$ for $\preceq$, which converges to some $z \in X$ with respect to $\tau_{M}$, it follows $x_{n} \preceq z$ for all $n \in \omega$.

Our main result in this section is the following.

Theorem 5.24. Let $(X, \preceq, M, *)$ be an ordered complete fuzzy metric space and $f: X \rightarrow X$ a nondecreasing mapping for $\preceq$, such that

$$
M(x, y, t)>1-t \Rightarrow M(f x, f y, \varphi(t))>1-\varphi(t)
$$

for all $x, y \in X$ with $x \preceq y$ and $t>0$, where $\varphi: \mathbb{R}^{+} \rightarrow \mathbb{R}^{+}$is a nondecreasing function satisfying $\lim _{n \rightarrow \infty} \varphi^{n}(t)=0$ for all $t>0$.

If there is $x_{0} \in X$ such that $x_{0} \preceq f x_{0}$, then $f$ has a fixed point $z \in X$. Moreover, the set of fixed points of $f$ is a singleton if and only it is wellordered.

Proof. Take $t_{0}>1$. Let $x, y \in X$ with $x \preceq y$. From $M\left(x, y, t_{0}\right)>1-t_{0}$, it follows that

$$
M\left(f x, f y, \varphi\left(t_{0}\right)\right)>1-\varphi\left(t_{0}\right)
$$

Since $f$ is nondecreasing, we have that $f^{n} x \preceq f^{n} y$ for alll $n \in \omega$, so, by (5.1) and (5.2), we immediately deduce that

$$
M\left(f^{n} x, f^{n} y, \varphi^{n}\left(t_{0}\right)\right)>1-\varphi^{n}\left(t_{0}\right)
$$

for all $x, y \in X$ with $x \preceq y$ and $n \in \omega$.

Now let $x_{0} \in X$ such that $x_{0} \preceq f x_{0}$. Put $x_{n}=f^{n} x$ for all $n \in \omega$. Since $f$ is nondecreasing it follows that $\left(x_{n}\right)_{n \in \omega}$ is a nondecreasing sequence for $\preceq$

Next we show that $\left(x_{n}\right)_{n \in \omega}$ is a Cauchy sequence in $(X, M, *)$. To this end, choose $\varepsilon \in(0,1)$ and $t>0$. Then, there exists $n_{\varepsilon} \in \mathbb{N}$ such that $\varphi^{n}\left(t_{0}\right)<\min \{\varepsilon, t\}$ for all $n \geq n_{\varepsilon}$. Let $m>n \geq n_{\varepsilon}$. Then $m=n+k$ for some 
$k \in \mathbb{N}$, so, by $(5.3)$,

$$
\begin{aligned}
M\left(x_{n}, x_{m}, t\right) & =M\left(f^{n} x_{0}, f^{n} f^{k} x_{0}, t\right) \\
& \geq M\left(f^{n} x_{0}, f^{n} f^{k} x_{0}, \varphi^{n}\left(t_{0}\right)\right) \\
& >1-\varphi^{n}\left(t_{0}\right) \\
& >1-\varepsilon .
\end{aligned}
$$

Therefore $\left(x_{n}\right)_{n \in \omega}$ is a Cauchy sequence in $(X, M, *)$. Since $(X, M, *)$ is complete there exists $z \in X$ such that $\lim _{n \rightarrow \infty} M\left(z, x_{n}, t\right)=1$ for all $t>0$.

It remains to show that $z$ is a fixed point of $f$ and that $z$ is the unique fixed point of $f$ if and only if the set of fixed points of $f$ is well-ordered.

Given $t>0$ and $\varepsilon \in(0, t)$ there exists $n_{\varepsilon} \in \mathbb{N}$ such that

$$
M\left(x_{n}, z, \varepsilon\right)>1-\varepsilon
$$

for all $n \geq n_{\varepsilon}$. Since $x_{n} \preceq z$ and $\varphi(\varepsilon)<\varepsilon<t$, we deduce from (5.1) that

$$
M\left(x_{n+1}, f z, t\right)>1-\varphi(\varepsilon)
$$

for all $n \geq n_{\varepsilon}$, so $M\left(x_{n+1}, f z, t\right)>1-\varepsilon$ for all $n \geq n_{\varepsilon}$.

We conclude that $\lim _{n \rightarrow \infty} M\left(f z, x_{n}, t\right)=1$ for all $t>0$. Hence, $z=f z$.

Finally, if the set of fixed point is well-ordered and $u \in X$ is a fixed point of $f$, we deduce by (5.3) and assuming $u \preceq z$, that

$$
M\left(f^{n} u, f^{n} z, \varphi^{n}\left(t_{0}\right)\right)>1-\varphi^{n}\left(t_{0}\right)
$$

for all $n \in \omega$, i.e., $M\left(u, z, \varphi^{n}\left(t_{0}\right)\right)>1-\varphi^{n}\left(t_{0}\right)$ for all $n \in \omega$. Given $\varepsilon \in(0,1)$ and $t>0$, there exists $k \in \mathbb{N}$ such that $\varphi^{k}\left(t_{0}\right)<\min \{\varepsilon, t\}$. Hence

$$
M(u, z, t) \geq M\left(u, z, \varphi^{k}\left(t_{0}\right)\right)>1-\varphi^{k}\left(t_{0}\right)>1-\varepsilon
$$

which implies that $M(u, z, t)=1$ for all $t>0$, and thus $u=z$. The converse is obvious. 
Remark 5.25. Theorem 5.24 provides an ordered counterpart of Theorem 5.3 and a fuzzy extension of Theorem 5.22.

The following easy example illustrates the usefulness of Theorem 5.24 with respect to Theorem 5.3.

Example 5.26. Let $(X, M, \wedge)$ be the complete fuzzy metric space where $X=\{a, b, c\}, M(a, a, t)=M(b, b, t)=M(c, c, t)=1$ for all $t>0$, and $M(x, y, t)=0$, otherwise. Define $f: X \rightarrow X$ as $f a=f c=a$ and $f b=c$, and let $\preceq$ be the (partial) order on $X$ given by

$$
x \preceq y \text { if and only if } x=a \text { and } y=c \text {, or } x=y \text {. }
$$

We show that the contraction condition of Theorem 5.3 does not hold for the complete fuzzy metric space $(X, M, *)$ and the map $f$. Indeed, assume the contrary. Then there is a nondecreasing function $\varphi: \mathbb{R}^{+} \rightarrow \mathbb{R}^{+}$ with $\lim _{n \rightarrow \infty} \varphi^{n}(t)=0$ for all $t>0$, and such that $M(f b, f c, \varphi(t))>$ $1-\varphi(t)$ whenever $t>1$, because $M(b, c, t)>1-t$ whenever $t>1$. Since $M(f b, f c, \varphi(t))=0$, it follows that $\varphi(t)>1$ whenever $t>1$, which contradicts that $\lim _{n \rightarrow \infty} \varphi^{n}(t)=0$. Therefore, we cannot apply Theorem 5.3 to this example.

However, since $a \preceq f a$, and condition (5.1) trivially holds since $M(f a, f c, t)=$ 1 for all $t>0$, the conditions of Theorem 5.24 are satisfied. In fact $a$ is the unique fixed point of $f$. 


\section{Chapter 6}

\section{The fuzzy quasi-metric case}

\subsection{Introduction}

The fact that the relation $\sqsubseteq_{d}$ defined by Edalat and Heckmann on the set of formal balls of a metric space $(X, d)$, preserves its structure of partial order in the case that $(X, d)$ is a quasi-metric space, suggests the natural and interesting question of extending the theory developed for metric spaces to the quasi-metric setting. This questions was discussed, among others, by Aliakbari, Honari, Pourmahdian and Rezaii ([5]), and Romaguera and Valero $([66,67])$.

The purpose of this chapter is to explore the extension and generalization of the main results obtained in Chapters 3,4 and 5, to the fuzzy quasi-metric framework.

\subsection{The results}

We start this section by discussing the problem of extending the main results of Chapter 3 to the quasi-metric framework. 
As in the fuzzy metric case, for a fuzzy quasi-metric space $(X, M, *)$ we define a binary relation $\sqsubseteq_{M}$ on the set $\mathbf{B} X:=X \times \mathbb{R}^{+}$of formal balls of $X$, by

$$
(x, r) \sqsubseteq_{M}(y, s) \Longleftrightarrow M(x, y, t) \geq \frac{t}{t+r-s} \quad \text { for all } t>0 .
$$

Note (compare Remark 3.2) that condition $(x, r) \sqsubseteq_{M}(y, s)$ implies $r \geq s$.

Then, we can generalize Proposition 3.3 as follows.

Proposition 6.1. Let $(X, M, \wedge)$ be a fuzzy quasi-metric space. Then $\left(\mathbf{B} X, \sqsubseteq_{M}\right)$ is a poset.

Proof. Since reflexivity and transitivity of $\sqsubseteq_{M}$ follows as in the fuzzy metric case (see the proof of Proposition 3.3), we only check the antisymmetry. Indeed, suppose $(x, r) \sqsubseteq_{M}(y, s)$ and $(y, s) \sqsubseteq_{M}(x, r)$. Then

$$
M(x, y, t) \geq \frac{t}{t+r-s} \quad \text { and } \quad M(y, x, t) \geq \frac{t}{t+s-r}
$$

for all $t>0$. Thus $r=s$, and consequently

$$
M(x, y, t)=M(y, x, t)=1
$$

for all $t>0$. Hence $x=y$.

However, the main result of Chapter 3 (Theorem 3.22) only admits a partial generalization to the quasi-metric setting.

To show this, we first extend the notion of a standard complete fuzzy metric space as follows.

Definition 6.2. A fuzzy quasi-metric space $(X, M, *)$ is called standard complete if every standard Cauchy sequence converges in $\left(X, M^{-1}, *\right)$, where 
a sequence $\left(x_{n}\right)_{n}$ in $X$ is said to be standard Cauchy if for each $\varepsilon \in(0,1)$ there exists $n_{0} \in \mathbb{N}$ such that

$$
M\left(x_{n}, x_{m}, t\right)>\frac{t}{t+\varepsilon},
$$

whenever $m \geq n \geq n_{0}$ and $t>0$.

Then, we can prove the following result.

Proposition 6.3. Let $(X, M, \wedge)$ be a fuzzy quasi-metric space. If $\left(\mathbf{B} X, \sqsubseteq_{M}\right.$ ) is a dcpo, then $(X, M, \wedge)$ is standard complete.

Proof. Let $\left(x_{n}\right)_{n}$ be a standard Cauchy sequence in $(X, M, \wedge)$. As in the proof of Lemma 3.19, choose $n_{1} \in \mathbb{N}$ such that

$$
M\left(x_{n_{1}}, x_{n}, t\right) \geq \frac{t}{t+2^{-2}},
$$

for all $n \geq n_{1}$ and $t>0$. Similarly, take $n_{2}>n_{1}$ such that

$$
M\left(x_{n_{2}}, x_{n}, t\right) \geq \frac{t}{t+2^{-3}},
$$

for all $n \geq n_{2}$ and $t>0$. Continuing this process, we construct a subsequence $\left(x_{n_{k}}\right)_{k}$ of $\left(x_{n}\right)$ such that

$$
M\left(x_{n_{k}}, x_{n_{k+1}}, t\right) \geq \frac{t}{t+2^{-(k+1)}},
$$

for all $k \in \mathbb{N}$ and $t>0$. Therefore $\left(x_{n_{k}}, 2^{-k}\right) \sqsubseteq_{M}\left(x_{n_{k+1}}, 2^{-(k+1)}\right)$ for all $k \in \mathbb{N}$.

Since $\left(\mathbf{B} X, \sqsubseteq_{M}\right)$ is a dcpo there is $(x, r) \in \mathbf{B} X$ such that $(x, r)=\sqcup D$, where $D=\left\{\left(x_{n_{k}}, 2^{-k}\right): k \in \mathbb{N}\right\}$. Clearly $r=0$, so $\left(x_{n_{k}}, 2^{-k}\right) \sqsubseteq_{M}(x, 0)$ for all $k \in \mathbb{N}$, and consequently

$$
M\left(x_{n_{k}}, x, t\right) \geq \frac{t}{t+2^{-k}},
$$


for all $k \in \mathbb{N}$ and $t>0$. Therefore $\lim _{k \rightarrow \infty} M\left(x_{n_{k}}, x, t\right)=1$ for all $t>0$. It immediately follows that, in fact, one has

$$
\lim _{n \rightarrow \infty} M\left(x_{n}, x, t\right)=1
$$

for all $t>0$. We conclude that $(X, M, \wedge)$ is standard complete.

The next example shows the unfortunately, and contrarily to the fuzzy metric case, the converse of Proposition 6.3 is not true.

Example 6.4. Let $\mathcal{A}$ be the family of all nonempty countable subsets of $\mathbb{R}$ and let $(M, \wedge)$ be the fuzzy quasi-metric on $\mathcal{A}$ given by $M(A, B, t)=1$ if $A \subseteq B$ and $M(A, B, t)=0$ otherwise. Clearly $(\mathcal{A}, M, \wedge)$ is standard complete because if $\left(A_{n}\right)_{n}$ is a standard Cauchy sequence then $A_{n} \subseteq A_{n+1}$ for all $n$, and

$$
\lim _{n \rightarrow \infty} M\left(A_{n}, \bigcup_{n=1}^{\infty} A_{n}, t\right)=1,
$$

for all $t>0$ (of course, $\bigcup_{n=1}^{\infty} A_{n} \in \mathcal{A}$ ).

However, the subset of $\mathbf{B} \mathcal{A}$

$\{(A, 0): A$ is a nonempty finite set consisting of irrational numbers $\}$ is directed but has no upper bound.

With respect to the results obtained in Chapter 4 , we notice that if $(X, M, *)$ is a fuzzy quasi-metric then we may define, as in the fuzzy metric case, a binary relation $\sqsubseteq$ on $\mathbf{B} X$ given by

$$
(x, r) \sqsubseteq(y, s) \Longleftrightarrow y \in \bigcap_{t>r-s} \bar{B}_{M}(x,(r-s) \wedge 1, t) .
$$

It is easily follows that

$$
(x, r) \sqsubseteq(y, s) \Longleftrightarrow \inf _{t>r-s} M(x, y, t) \geq 1-((r-s) \wedge 1)
$$


In fact, Proposition 4.2 can be generalized to fuzzy quasi-metric spaces.

Proposition 6.5. Let $(X, M, *)$ be a fuzzy quasi-metric space with $* \geq$ $*_{L}$. Then $(\mathbf{B} X, \sqsubseteq)$ is a poset.

Proof. Since reflexivity and transitivity follow exactly as in the proof of Proposition 4.2 , we only verify the antisymmetry of $\sqsubseteq$.

Let $(x, r) \sqsubseteq(y, s)$ and $(y, s) \sqsubseteq(x, r)$. Then $r=s$, and thus

$$
\inf _{t>0} M(x, y, t) \geq 1 \quad \text { and } \quad \inf _{t>0} M(y, x, t) \geq 1
$$

i.e., $M(x, y, t)=M(y, x, t)=1$ for all $t>0$, so $x=y$.

Furthermore, the proof of Proposition 4.3 shows that this result also admits a natural generalization.

Proposition 6.6. Let $(X, d)$ be a metric space with $d \leq 1$. Then $\sqsubseteq_{d}=\sqsubseteq$ on $\mathbf{B} X$, where $\sqsubseteq$ is the partial order on $\mathbf{B} X$ corresponding to the fuzzy quasimetric space $\left(X, M_{01}, *\right), * \geq *_{L}$.

It is well known (see e.g. [13]) that given a fuzzy quasi-metric space $(X, M, *)$ with $* \geq *_{L}$, then the function $d(M)$ defined by

$$
d(M)(x, y)=\sup \{t \geq 0: M(x, y, t) \leq 1-t\}
$$

is a quasi-metric on $X$, with $d(M) \leq 1$, whose induced topology coincides with the topology induced by $(M, *)$. In fact, for each $x, y \in X$ and each $\varepsilon \in(0,1)$ we have

$$
M(x, y, \varepsilon)>1-\varepsilon \Longleftrightarrow d(M)(x, y)<\varepsilon,
$$

Then, we can deduce the following result (compare Corollary 4.6). 
Proposition 6.7. Let $(X, M, *)$ be a fuzzy quasi-metric space with $* \geq *_{L}$. Then $(X, M, *)$ is bicomplete if and only if $(X, d(M))$ is bicomplete.

Moreover, the proof of Proposition 4.7 allows its generalization to the quasi-metric framework as follows.

Proposition 6.8. Let $(X, M, *)$ be a fuzzy quasi-metric space with $* \geq *_{L}$. Then $\sqsubseteq=\sqsubseteq d(M)$ on $\mathbf{B} X$.

In Theorem 4.9 it was proved that a fuzzy metric space $(X, M, *)$ is complete if and only if $(\mathbf{B} X, \sqsubseteq)$ is a domain.

Next we show that this crucial result cannot be generalized to the quasimetric case. To this end we shall use Example 6.4 above.

Let $\mathcal{A}$ be the family of all nonempty countable subsets of $\mathbb{R}$ and let $(M, \wedge)$ be the fuzzy quasi-metric on $\mathcal{A}$ given by $M(A, B, t)=1$ if $A \subseteq B$ and $M(A, B, t)=0$ otherwise. Clearly $(\mathcal{A}, M, \wedge)$ is bicomplete because if $\left(A_{n}\right)_{n}$ is a Cauchy sequence in $\left(\mathcal{A}, M^{i}, \wedge\right)$, it is eventually constant.

However, as in Example 6.4, the subset of $\mathbf{B} \mathcal{A}$

$$
\{(A, 0): A \text { is a nonempty finite set consisting of irrational numbers }\} \text {, }
$$

is directed in $(\mathbf{B} \mathcal{A}, \sqsubseteq)$ but has no upper bound.

Despite this, it is still possible to recover to the quasi-metric context some results of Theorem 4.12 .

In fact, if $(X, M, *)$ is a fuzzy quasi-metric space with $* \geq *_{L}$, the result (1) of Theorem 4.12 remains valid, so we have that $\left(\mathcal{M}, *_{L}\right)$ is a fuzzy quasimetric on $\mathbf{B} X$, where 


$$
\mathcal{M}((x, r),(y, s), 0)=0,
$$

for all $(x, r),(y, s) \in \mathbf{B} X, \quad$ and

$\mathcal{M}((x, r),(y, s), t)=\left[\left(M\left(x, y,(t+r-s)^{+}\right) \wedge(1-(|r-s| \wedge 1))\right)+((r-s) \wedge 1)\right]^{+}$

for all $(x, r),(y, s) \in \mathbf{B} X$ and $t>0$.

We also can show, with an adaptation of the corresponding proofs in Theorem 4.12, the following facts:

(a) $\sqsubseteq=\leq_{\mathcal{M}}$ on $\mathrm{B} X$.

(b) $(X, M, *)$ and $\left(\mathbf{B} X_{0}, \mathcal{M}, *_{L}\right)$ are isometric, where $\mathbf{B} X_{0}=\{(x, 0)$ : $x \in X\}$.

(c) $(X, M, *)$ is complete if and only if $\left(\mathbf{B} X, \mathcal{M}, *_{L}\right)$ is bicomplete.

We conclude by deducing from the main theorem of Chapter 5 a quasimetric version of it.

Proposition 6.9. Let $(X, M, *)$ be a bicomplete fuzzy quasi-metric space and $f: X \rightarrow X$ a map such that

$$
M(x, y, t)>1-t \Rightarrow M(f x, f y, \varphi(t))>1-\varphi(t)
$$

for all $x, y \in X$ and $t>0$, where $\varphi: \mathbb{R}^{+} \rightarrow \mathbb{R}^{+}$is a nondecreasing function satisfying $\lim _{n \rightarrow \infty} \varphi^{n}(t)=0$ for all $t>0$. Then $f$ has a unique fixed point.

Proof. Let $x, y \in X$ and $t>0$ such that $M^{i}(x, y, t)>1-t$, then $M(x, y, t)>1-t$ and $M(y, x, t)>1-t$. Therefore

$$
M(f x, f y, \varphi(t))>1-\varphi(t) \text { and } \quad M(f y, f x, \varphi(t))>1-\varphi(t)
$$


SO

$$
M^{i}(f x, f y, \varphi(t))>1-\varphi(t) .
$$

Hence, all conditions of Theorem 5.3 are satisfied for the complete fuzzy metric space $\left(X, M^{i}, *\right)$, and consequently, $f$ has a unique fixed point. 


\section{Bibliography}

[1] M. Abbas, M. Imdad, D. Gopal, $\psi$-weak contractions in fuzzy metric spaces, Iranian J. Fuzzy Syst. 8 (2011), 141-148.

[2] S. Abramsky, A. Jung, Domain Theory, in: Handbook of Logic in Computer Science, vol. 3, pp. 1-168. Clarendon Press, 1994.

[3] C. Alaca, D. Turkoglu, C. Yildiz, Fixed points in intuitionistic fuzzy metric spaces, Chaos Soliton Fract. 29 (2006), 1073-1078.

[4] B. Ali, M. Abbas, Suzuki-type fixed point theorem for fuzzy mappings in ordered metric spaces, Fixed Point Theory Appl. 2013, 2013 : 9.

[5] M. Aliakbari, B. Honari, M. Pourmahdian, M.M. Rezaii, The space of formal balls and models of quasi-metric spaces, Math. Struct. Comput. Sci. 19 (2009), 337-355.

[6] I. Altun, Some fixed point theorems for single and multi valued mappings on ordered nonarchimedean fuzzy metric spaces, Iranian J. Fuzzy Syst. 7 (2010), 91-96.

[7] I. Altun, D. Mihet, Ordered non-Archimedean fuzzy metric spaces and some fixed point results, Fixed Point Theory Appl. 2010 (2010), Article ID 782680, 11 pages. 
[8] I. Altun, H. Simsek, Some fixed point theorems on ordered metric spaces and application, Fixed Point Theory Appl. 2010 (2010), Article ID 621469, 17 pages.

[9] R.P. Agarwal, M.A. El-Gebeily, D. O'Regan, Generalized contractions in partially ordered metric spaces, Appl. Anal. 87 (2008), 109-116.

[10] J. de Bakker, E. de Vink, Control Flow Semantics, The MIT Press, Cambridge, 1996.

[11] T.G. Bhaskar, V. Lakshmikantham, Fixed point theorems in partially ordered metric spaces and applications, Nonlinear Anal. TMA, 65 (2006), 1379-1393.

[12] D. W. Boyd, J. S. W. Wong, On nonlinear contractions, Proc. Am. Math. Soc. 20 (1969), 458-464.

[13] F. Castro-Company, S. Romaguera, P. Tirado, The bicompletion of fuzzy quasi-metric spaces, Fuzzy Sets Syst. 166 (2011), 56-64.

[14] Y.J. Cho, M. Grabiec, V. Radu, On Nonsymmetric Topological and Probabilistic Structures, Nova Science Publisher, Inc., NewYork, 2006.

[15] L.B. Ćirić, Solving the Banach fixed point principle for nonlinear contractions in probabilistic metric spaces, Nonlinear Anal. TMA. 72 (2010), 2009-2018.

[16] Lj. B. Ćirić, N. Cakić, M. Rajović, J.S. Ume, Monotone generalized nonlinear contractions in partially ordered metric spaces, Fixed Point Theory Appl. 2008 (2008), Article ID 131294, 11 pages.

[17] L.B. Ćirić, D. Mihet, R. Saadati, Monotone generalized contractions in partially ordered probabilistic metric spaces, Topology Appl. 156 (2009), 2838-2844. 
[18] A. Edalat, Domain theory and integration, Theor. Comput. Sci. 151 (1995), 163-193.

[19] A. Edalat, Dynamical systems, measures and fractals via domain theory, Inform. Comput. 120 (1995), 32-48.

[20] A. Edalat, R. Heckmann, A computational model for metric spaces, Theor. Comput. Sci. 193 (1998), 53-73.

[21] A. Edalat, Ph. Sünderhauf, Computable Banach spaces via domain theory, Theor. Comput. Sci. 219 (1999), 169-184.

[22] P. Fletcher, W.F. Lindgren, Quasi-Uniform Spaces, Marcel Dekker, New York, 1982.

[23] A. George, P. Veeramani, On some results in fuzzy metric spaces, Fuzzy Sets Syst. 64 (1994), 395-399.

[24] A. George, P. Veeramani, On some results of analysis of fuzzy metric spaces, Fuzzy Sets Syst. 90 (1997), 365-368.

[25] G. Gierz, K.H. Hofmann, K. Keimel, J.D. Lawson, M. Mislove, D.S. Scott, A Compendium of Continuous Lattices, Springer Verlag, Berlin, 1980.

[26] G. Gierz, K.H. Hofmann, K. Keimel, J.D. Lawson, M. Mislove, D.S. Scott, Continuous Lattices and Domains, Encyclopedia of Mathematics and its Applications 93, Cambridge University Press, 2003.

[27] I. Golet, On contractions in probabilistic metric spaces, Radovi Mat. 13 (2004), 87-92.

[28] M. Grabiec, Fixed points in fuzzy metric spaces, Fuzzy Sets Syst. 27 (1988), 385-389. 
[29] V. Gregori, S. Morillas, A. Sapena, Examples of fuzzy metric spaces, Fuzzy Sets Syst. 170 (2011), 95-111.

[30] V. Gregori, S. Romaguera, P. Veeramani, A note on intuitionistic fuzzy metric spaces, Chaos Soliton. Fract. 28 (2006), 902-905.

[31] V. Gregori, S. Romaguera, Some properties of fuzzy metric spaces, Fuzzy Sets Syst. 115 (2000), 485-489.

[32] V. Gregori, S. Romaguera, Fuzzy quasi-metric spaces, Appl. Gen. Top. 5 (2004), 129-136.

[33] V. Gregori, A. Sapena, On fixed-point theorems in fuzzy metric spaces, Fuzzy Sets Syst. 125 (2002), 245-253.

[34] J. Gutiérrez García, S. Romaguera, Examples of non-strong fuzzy metrics, Fuzzy Sets Syst. 162 (2011), 91-93.

[35] O. Hadžić, Some theorems on the fixed points in probabilistic metric and random normed spaces, Boll. Unione Mat. Ital. B 6 (1982), 381-391.

[36] R. Heckmann, Approximation of metric spaces by partial metric spaces, Appl. Cat. Struct. 7 (1999), 71-83.

[37] T. L. Hicks, Fixed point theory in probabilistic metric spaces, Zb. Rad. Prir. Mat. Fak. Novi Sad, 13 (1983), 63-72.

[38] X. Huang, C. Zhu, X. Wen, On $(\mathrm{g}, \varphi)$-contraction in intuitionistic fuzzy metric spaces, Math. Commun. (2010), 425-435.

[39] J. Jachymski, Equivalent conditions for generalized contractions on (ordered) metric spaces, Nonlinear Anal. TMA. 74 (2011), 768-774.

[40] J. Jachymski, On probabilistic $\varphi$ - contractions on Menger spaces, Nonlinear Anal. TMA. 73 (2010), 2199-2203. 
[41] R. Kopperman, H.P. Künzi, P. Waszkiewicz, Bounded complete models of topological spaces, Topology Appl. 139 (2004), 285-297.

[42] I. Kramosil, J. Michalek, Fuzzy metrics and statistical metric spaces, Kybernetika 11 (1975), 326-334.

[43] H.P.A. Künzi, Nonsymmetric distances and their associated topologies: about the origins of basic ideas in the area of asymmetric topology, in: C.E. Aull, R. Lowen (Eds.), Handbook of the History of General Topology, vol. 3, Kluwer, Dordrecht, 2001, pp. 853-968.

[44] J. Lawson, Spaces of maximal points, Math. Struct. Comput. Sci. 7 (1997), 543-556.

[45] K. Martin, Domain theoretic models of topological spaces, Elect. Notes Theor. Comput. Sci. 13 (1998), 173-181.

[46] J. Matkowski, Integrable solutions of functional equations, Diss. Math. 127 (1975), 1-68.

[47] D. Mihet, A Banach contraction theorem in fuzzy metric spaces, Fuzzy Sets Syst. 144 (2004), 431-439.

[48] D. Mihet, A note on a paper of I. Golet, Sarajevo J. Math. 2 (2006), 115-117.

[49] D. Mihet, Fuzzy $\psi$-contractive mappings in non-Archimedean fuzzy metric spaces, Fuzzy Sets Syst. 159 (2008), 739-744.

[50] J.J. Nieto, R. Rodríguez-López, Contractive mapping theorems in partially ordered sets and applications to ordinary differential equations, Order 22(2005), 223-239.

[51] D. O'Regan, A. Petruşel, Fixed point theorems for generalized contractions in ordered metric spaces, J. Math. Anal. Appl. 341 (2008), 12411252 . 
[52] J. H. Park, Intuitionistic fuzzy metric spaces, Chaos Soliton. Fract. 22 (2004), 1039-1046.

[53] V. Radu, Some fixed point theorems in probabilistic metric spaces, Lecture Notes Math. 1233 (1987), 125-133.

[54] V. Radu, Some remarks on the probabilistic contractions on fuzzy Menger spaces, The Eighth Internat. Conf. on Applied Mathematics and Computer Science, Cluj-Napoca 2002, Automat. Comput. Appl. Math. 11 (2002), 125-131.

[55] A.C.M. Ran, M.C.B. Reurings, A fixed point theorem in partially ordered sets and some applications to matrix equations, Proc. Amer. Math. Soc. 132 (2004), 1435-1443.

[56] L.A. Ricarte, S. Romaguera, The set of formal balls of a complete fuzzy metric space viewed as a continuous domain, in: Applied Topology: Recent Progress for Computer Science, Fuzzy Mathematics and Economics, Proceedings of the Workshop in Applied Topology WiAT'12, Col.lecció Treballs d'Informàtica i Tecnologia, Universitat Jaume I, vol. 40, 2012, pp. 169-175.

[57] L.A. Ricarte, S. Romaguera, A domain-theoretic approach to fuzzy meric spaces, Topology Appl., accepted.

[58] L.A. Ricarte, S. Romaguera, On $\varphi$-contractions in fuzzy metric spaces with application to the intuitionistic setting, Iranian J. Fuzzy Syst., accepted.

[59] L.A. Ricarte, S. Romaguera, P. Tirado, A fixed point theorem for generalized contractions on ordered complete fuzzy metric spaces, in: Recent Progress for Computer Science, Fuzzy Mathematics and Economics, Proceedings of the Conference in Applied Topology WiAT'13, University of the Basque Country, 2013, pp. 109-114. 
[60] L.A. Ricarte, S. Romaguera, A quantitative computational model for complete fuzzy metric spaces, submitted.

[61] S. Romaguera, On Computational Models for the Hyperspace, Advances in Mathematics Research, volume 8, Nova Science Publishers, New York, pp. 277-294. 2009.

[62] S. Romaguera, M.A. Sánchez Granero, M. Sanchis, On the construction of domains of formal balls for uniform spaces, Topology Appl., accepted.

[63] S. Romaguera, M.P. Schellekens, O. Valero, Complexity spaces as quantitative domains of computation, Topology Appl. 158 (2011), 853-860.

[64] S. Romaguera, P. Tirado, On fixed point theorems in intuitionistic fuzzy metric spaces, Internat. J. Nonlinear Sci. Numer. Simul. 8 (2007), 233238.

[65] S. Romaguera, P. Tirado, Contraction maps on ifqm-spaces with application to recurrence equations of Quicksort, Electronic Notes Theoret. Comput. Sci. 225 (2009), 269-279.

[66] S. Romaguera, O. Valero, A quantitative computational model for complete partial metric spaces via formal balls, Math. Struct. Comput. Sci. 19 (2009), 541-563.

[67] S. Romaguera, O. Valero, Domain theoretic characterisations of quasimetric completeness in terms of formal balls, Math. Struct. Comput. Sci. 20 (2010), 453-472.

[68] J.J.M.M. Rutten, Weighted colimits and formal balls in generalized metric spaces, Topology Appl. 89 (1998), 179-202.

[69] R. Saadati, J.H. Park, On the intuitionistic fuzzy topological spaces, Chaos Soliton. Fract. 27 (2006), 331-344. 
[70] R. Saadati, S. Sedghi, H. Zaou, A common fixed point theorem for $\psi$ weakly commuting maps in L-fuzzy metric spaces, Iranian J. Fuzzy Syst. 5 (2008), 47-53.

[71] M.P. Schellekens, A characterization of partial metrizability. Domains are quantifiable, Theor. Comput. Sci. 305 (2003), 409-432.

[72] B. Schweizer, A. Sklar, Statistical metric spaces, Pacific J. Math. 10 (1960), 314-334.

[73] B. Schweizer, A. Sklar, Probabilistic Metric Spaces, Dover Books on Mathematics, Dover, New York, 2005(first edition: 1983).

[74] D. Scott, C. Strachey, Toward a mathematical semantics for computer languages, Oxford Programming Research Group Technical Monograph. PRG-6. 1971.

[75] V. Stoltenberg-Hansen, I. Lindström, E.R. Griffor, Mathematical Theory of Domains, Cambridge Tracts in Theoretical Computer Science, vol. 22, Cambridge University Press, 1994.

[76] P. Tirado, On compactness and G-completeness in fuzzy metric spaces, Iranian J. Fuzzy Syst., 9 (2012), 151-158.

[77] R. Vasuki, P. Veeramani, Fixed point theorems and Cauchy sequences in fuzzy metric spaces, Fuzzy Sets Syst. 135 (2003), 409-413.

[78] P. Waszkiewicz, Quantitative continuous domains, Applied Cat. Struct. 11 (2003), 41-67.

[79] P. Waszkiewicz, Partial metrisability of continuous posets, Math. Struct. Comput. Sci. 16 (2006), 359-372. 